\title{
Radio-loud AGN in the XMM-LSS field
}

\section{Optical identification and sample selection ${ }^{\star}$}

\author{
C. Tasse ${ }^{1}$, D. Le Borgne ${ }^{2}$, H. Röttgering ${ }^{1}$, P. N. Best ${ }^{3}$, M. Pierre $^{2}$, and B. Rocca-Volmerange ${ }^{4}$ \\ 1 Leiden Observatory, University of Leiden, PO Box 9513, 2300 RA Leiden, The Netherlands \\ e-mail: tasse@strw. leidenuniv.nl \\ 2 CEA/DSM/DAPNIA, Service d'Astrophysique, Saclay, 91191 Gif-sur-Yvette, France \\ 3 SUPA, Institute for Astronomy, Royal Observatory Edinburgh, Blackford Hill, Edinburgh EH9 3HJ, UK \\ ${ }^{4}$ Institut d'Astrophysique de Paris and Université Paris-Sud, Orsay, France
}

Received 9 August 2007 / Accepted 11 May 2008

ABSTRACT

\begin{abstract}
The XMM-Large Scale Structure survey field (XMM-LSS) is an extragalactic window surveyed in the X-ray with the XMM-Newton satellite. It has also been observed in the optical with the Canada-France Hawaï Telescope (CFHTLS survey), and in the infrared with the Spitzer Space Telescope (SWIRE survey). These surveys have been carried out to study the structure and evolution of both baryonic and dark matter on cosmological scales. In two previous papers, we presented deep low frequency radio surveys of the XMM-LSS field, with limiting flux density levels of $\sim 4$ and $\sim 1.5 \mathrm{mJy} /$ beam at 325 and $610 \mathrm{MHz}$ respectively $(5 \sigma)$. These radio surveys were motivated by the need to understand the various connections between the host galaxies of radio sources and their environments.

In this paper, we identify optical counterparts to the low frequency radio sources, using the CFHTLS optical catalogue and images, that have an $i$-band limiting magnitude of $i_{\mathrm{AB}} \sim 25$. We use a likelihood ratio method and estimate that $\sim 75 \%$ of the radio sources have a detected optical counterpart. Using the CFHTLS and SWIRE data, we derive photometric redshifts for the galaxies that are identified with a radio source, as well as for those that are not; we demonstrate the reliability of these photometric redshifts by deriving the stellar mass function for galaxies at different redshifts, and showing that it is consistent with previous determinations. We classify the radio sources as type-1 AGN, radio galaxies, or star-forming galaxies, and show that the radio luminosity function of the radio galaxies agrees with previous measurements.
\end{abstract}

Key words. methods: observational - techniques: photometric - galaxies: active - galaxies: fundamental parameters cosmology: observations - radio continuum: galaxies

\section{Introduction}

With the availability of large surveys, it has now become possible to study in great detail the relationship between the various classes of active galactic nuclei (AGN), their host galaxies and their environments (see Heckman \& Kauffmann 2006, for a review). Recent findings indicate that the criteria used to select AGN have a significant influence on the observed properties of the AGN population. In the local $z \lesssim 0.3$ universe, AGN selected using optical emission line criteria have rather high accretion rates, and are preferentially situated in fairly massive galaxies (Kauffmann et al. 2003; Heckman et al. 2004). Quite strikingly, it appears that samples of AGN selected by radio luminosity are statistically independent, at low radio power, $\left(P_{1.4 \mathrm{GHz}}<10^{25} \mathrm{~W} \mathrm{~Hz}^{-1}\right)$, from samples of emission-line selected AGN (Best et al. 2005) (i.e. the probability of galaxy being classified as a low-power radio emitting AGN does not depend on whether it is classified as an emission-line AGN or not). This suggests that those two populations are fundamentally different.

Many authors have noted that the properties of these lowpower radio-loud are very different to those generally seen in AGN. Specifically, Hine \& Longair (1979) observed that many

\footnotetext{
* Appendices D and E are only available in electronic form at http///www . aanda.org
}

low power radio galaxies (and also some higher power sources) do not have the luminous narrow emission lines expected in the framework of the unified scheme (see also Laing et al. 1994; Jackson \& Rawlings 1997); these are often referred to as lowexcitation radio galaxies (LERGs). In addition the expected infrared emission from a dusty torus is in general not observed (Whysong \& Antonucci 2004; Ogle et al. 2006) and nor is accretion-related X-ray emission (Hardcastle et al. 2006; Evans et al. 2006). Combined, these arguments suggest that these low power radio sources are a distinct type of AGN phenomenon, accreting through a radiatively inefficient mode (sometimes called the "Radio mode"), rather than the radiatively efficient accretion mode of optically or X-ray selected AGN (the so-called "Quasar mode").

The physical reasons for the origin of these two accretion modes are still unclear. It has been suggested that the quasar mode is produced by the accretion of cold gas onto the supermassive black hole, while the accretion of hot gas might drive a radiatively inefficient accretion (see Hardcastle et al. 2007, for a discussion). These two accretion modes might be related to the nature of the process that brings the gas to the central supermassive black-hole. In that framework, galaxy mergers or interactions trigger a radiatively-efficient accretion of cold gas. In contrast, the slow accretion of the hot intergalactic medium gas, that is seen to be cooling in the atmosphere of massive elliptical 
galaxies (see Mathews \& Brighenti 2003; Best et al. 2005, and references therein), occurs in a radiatively inefficient mode. A good way of testing this scenario, in which the nature of the triggering process drives the accretion type, is to study the properties and environment of quasar mode and radio mode AGN.

The XMM-Large Scale Structure (XMM-LSS) field has been surveyed at low radio frequencies, and in the infrared, optical, UV, and X-ray wavebands over $\sim 10 \mathrm{deg}^{2}$ (for a general presentation of the associated surveys see Pierre et al. 2004). This combination of data is well-suited for testing the possible link between triggering process and accretion mode: the midinfrared data may provide information on the presence of hot dust (radiatively efficient accretion), while the combination of width and depth of the optical data allows for a detailed study of the influence of the environment on the AGN activity, that might constrain the nature of the triggering mechanisms. In this paper, we build a sample of radio-loud AGN that may contain both quasar and radio mode AGN. In the next paper of the series, we will study their properties including the dependence of radio-loud AGN activity on both stellar mass and environment, down to low stellar mass $10^{9-10} M_{\odot}$ ).

In Sect. 2 we briefly present the radio and optical data used throughout this paper. We identify the optical and infrared counterparts of the VLA and GMRT low frequency radio sources in Sect. 3. By using the stellar synthesis code ZPEG, in Sect. 4 we estimate some of the properties of galaxies such as redshift, stellar mass, and star formation rate. These estimates will only be accurate for those radio galaxies whose emission is dominated by stellar light (the type-2 AGN), while they may be corrupted for the broad-line, core-dominated type-1 AGN. Therefore in Sect. 5 we proceed to a careful classification of the radio source host galaxies. The uncertainties on the estimated physical parameters are discussed in Sect. 6. We show that the stellar mass function of normal galaxies and the radio luminosity function of radio-loud AGN agree with previous measurements (Sect. 6.3). In the next paper of the series (Tasse et al. 2008), we will present the environmental estimator, and discuss the results of our study.

\section{Surveys of the XMM-LSS field}

\subsection{VLA radio data at 74 and $325 \mathrm{MHz}$}

We use the radio data described in detail in Tasse et al. (2006). The observations were carried out using the Very Large Array (VLA) at 74 and $325 \mathrm{MHz}$ simultaneously (4P mode). The radio survey consists of four pointings, observed in June, July and August 2003 in the A configuration and in June and July 2002 in the B configuration (see Fig. 1). The total integration time was $\sim 60 \mathrm{~h}$, split over the 4 pointing centers. The extended A configuration provides the high angular resolution needed for the optical identification work, whereas the B configuration is necessary to detect low surface brightness radio emission. Great attention has been paid to properly calibrate any corrupting influence of the ionosphere (Cohen et al. 2003).

The different pointings have been assembled into single maps covering $\sim 130 \mathrm{deg}^{2}$ and $\sim 15 \mathrm{deg}^{2}$ at 74 and $325 \mathrm{MHz}$ respectively. Since the noise is highly variable over the fields, we extracted the sources in the maps normalised by the local noise.

At $325 \mathrm{MHz}$, we have an angular resolution of 6.7", a median $5 \sigma$ sensitivity limit of $4.0 \mathrm{mJy} /$ beam, and we detect $\sim 850$ sources $(>5 \sigma)$. At $74 \mathrm{MHz}$, the angular resolution is $30^{\prime \prime}$, with a $5 \sigma$ sensitivity limit of $\sim 160 \mathrm{mJy} /$ beam, and we detect 650 sources. The positional accuracy at $325 \mathrm{MHz}$ of $\sim 2$ " is good enough for the optical identification of radio sources.

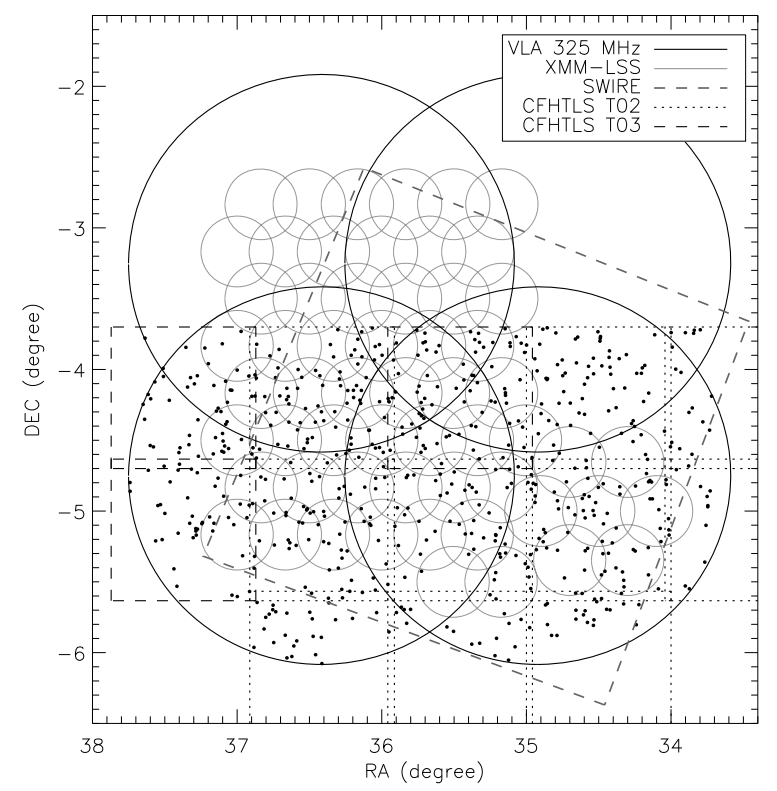

Fig. 1. The location of the various available surveys in the XMM-LSS field. The thin, grey circles show the positions of the observed XMM X-ray pointings. The four solid thick circles show the $325 \mathrm{MHz}$ pointings observed with the VLA, and the dots are at the positions of the sources detected at $325 \mathrm{MHz}$ (Tasse et al. 2006) that overlap with the coverage of the CFHTLS-Wide optical data; these optical observations are indicated by the dotted lines for the T02 release and dashed lines for the T03 release. The SWIRE field is indicated by dashed lines.

\subsection{GMRT radio data at 230 and $610 \mathrm{MHz}$}

Low-frequency radio observations of the XMM-LSS have been carried out with the Giant Meterwave Radio Telescope (GMRT) at 230 and $610 \mathrm{MHz}$ (Tasse et al. 2007). These data provide two additional flux density measurements for $41 \%$ of the radio sources detected at $325 \mathrm{MHz}$ with the VLA. These observations were motivated by the need to study the influence of the optical host galaxy and environmental properties on the observed radio spectra for large samples of radio sources.

At $610 \mathrm{MHz}$, the sky coverage is $12.7 \mathrm{deg}^{2}$, reaching an average noise level of $\sim 0.3 \mathrm{mJy} / \mathrm{beam}$; this leads to the detection of 767 sources. At $230 \mathrm{MHz}$, we reach an average noise level of $\sim 2.5 \mathrm{mJy} /$ beam, detecting 467 radio sources over $\sim 18.0 \mathrm{deg}^{2}$. The positional accuracies are typically $3^{\prime \prime}$ and $2^{\prime \prime}$ at 230 and $610 \mathrm{MHz}$ respectively.

\subsection{CFHTLS-W1 optical data}

The aim of the Wide component of the Canada France Hawaii Telescope Legacy Survey ${ }^{1}$ (CFHTLS) is to cover 170 squaredegrees spread over 3 areas of the sky (W1, W2, W3). The W1 field patch covers $7 \times 7$ square degrees and is centered at $\alpha(\mathrm{J} 2000)=02^{\mathrm{h}} 18^{\mathrm{m}} 00^{\mathrm{s}}, \delta(2000)=-07^{\circ} 00^{\prime} 00^{\prime \prime}$, partially covering the XMM-LSS field (Fig. 1). The observations are carried out through queued service observing, using the 1 degree $^{2}$ MegaCam CCD detector. Typical exposure times are $\sim 1 \mathrm{~h}$ in each $u^{*}, g^{\prime}, r^{\prime}, i^{\prime}$ and $z^{\prime}$ bands, leading to a limiting magnitude of $i_{\mathrm{AB}} \sim 25$. The observations were carried out between June 1 , 2003 and Sept. 12, 2005. Of the $13 \mathrm{deg}^{2}$ of CFHTLS data used throughout this paper, 10 were part of the Terapix T02 release,

1 http://www.cfht.hawaii.edu/Science/CFHLS/ 
and the 3 were part of the Terapix T03 release (see Fig. 1). The $13 \mathrm{deg}^{2}$ catalog contains $\sim 3 \times 10^{6}$ objects.

The $u^{*} g^{\prime} r^{\prime} i^{\prime} z^{\prime}$ magnitudes and associated error bars are Kron-like (Kron 1980; Bertin \& Arnouts 1996), in the AB magnitude system (Oke \& Gunn 1983). Also, for each object the Terapix catalog contains a flag indicating whether a source:

- is extended or point-like. This flag is extracted from the SExtractor "flux-radius" parameter, which measures the radius enclosing $50 \%$ of the flux. This classification cannot be applied to objects fainter than $i=21$, due to low signal-tonoise.

- is masked or not. The masks are built on the basis of the $i$-band images, and their role is to reject the field boundary, regions surrounding saturated stars, satellite tracks, and image defects.

- is saturated or not. An object is flagged as saturated when its $i$-band magnitude satisfies $i<17.8$.

The masks have been optimized for weak lensing studies. They are therefore very restrictive. On the basis of the $i$-band images we have redesigned new, less restrictive masks by suppressing the less relevant Terapix mask flags. The regions which have been unmasked are mainly the ones situated between the 36 CCDs of the MegaCam detector. We have changed the masking flag in the catalog accordingly. The new masks typically mask $\sim 20-25 \%$ of the total surveyed area, against $\sim 50 \%$ for the original Terapix masks.

\subsection{SWIRE survey data}

The Spitzer Wide-area InfraRed Extragalactic legacy survey (SWIRE, Lonsdale et al. 2003) is a $\sim 50 \mathrm{deg}^{2}$ high galactic latitude imaging survey. The SWIRE survey is spread over 6 regions observed in 4 bands with the IRAC instrument from 3.6 to $8.0 \mu \mathrm{m}$ and in 3 bands with MIPS from 24 to $160 \mu \mathrm{m}$. Spitzer observed $9.1 \mathrm{deg}^{2}$ of the XMM-LSS field as part of the SWIRE legacy survey in July 2004 (See Fig. 1). Throughout this paper we have used the data release 2 (DR2 hereafter) band-merged catalog available online ${ }^{2}$, containing the flux density measurements at 3.6, 4.5, 5.8, 8.0 and $24 \mu \mathrm{m}$ for a total of $\sim 2.5 \times 10^{5} \mathrm{ob}-$ jects. This catalog contains the sources detected above $5 \sigma$ from 3.6 to $8.0 \mu \mathrm{m}$ and above $3 \sigma$ at $24 \mu \mathrm{m}$, corresponding to limiting flux densities of $14,15,42,56$, and $280 \mu \mathrm{Jy}$ respectively, and positional accuracies better than $0.5^{\prime \prime}(2 \sigma)$. Following RowanRobinson et al. (2005), we have used the Kron flux density estimates for the IRAC bands for objects brighter than $S(3.6)_{\text {Kron }}>$ $1 \mathrm{mJy}$, and the aperture flux density for the fainter objects as well as for all the objects at $24 \mu \mathrm{m}$. The data reduction and quality assessment is extensively discussed in Surace et al. (2004).

\subsection{Field selection}

The optical T02/T03 and infrared DR2 data do not entirely cover the radio maps of the XMM-LSS field (Fig. 1). Since we want to derive photometric redshifts for $z \lesssim 1$ radio source hosts, the availability of $u^{*} g^{\prime} r^{\prime} i^{\prime} z^{\prime}$ optical data is of primary importance. We therefore selected from the original multifrequency radio catalog (Tasse et al. 2007) only the radio sources located in an area covered by at least three optical bands. In addition we restricted our study to the area covered by the $325 \mathrm{MHz}$ data. This

\footnotetext{
${ }^{2}$ See http://swire.ipac.caltech.edu/swire/ for more information.
}

means that the final sample contains 603 radio sources, corresponding to about $50 \%$ of the original radio catalogue. We notice that all sources are detected at 325 and/or $610 \mathrm{MHz}$, whereas no source is detected at 74 or $230 \mathrm{MHz}$ only, due to the higher flux density limit characterizing those radio surveys.

\section{Optical and infrared counterparts of radio sources}

We quantify the probability of an $i^{\prime}$-band optical object being the true host of a given radio source by using the likelihood ratio method which was first described by Richter (1975), and subsequently modified by de Ruiter et al. (1977), Prestage \& Peacock (1983), Benn (1983), and Wolstencroft et al. (1986). The version of the likelihood ratio method we use in this paper allows us to derive, for each optical candidate, an association probability that potentially takes into account its magnitude, location, colour, etc. (Sutherland \& Saunders 1992).

Prior to the likelihood ratio estimate, we carry out a visual inspection of the radio sources overlaid upon the $i^{\prime}$-band images, and classify their radio morphologies into different classes (Sect. 3.1). In Sect. 3.2, we describe the likelihood ratio method and, based on the magnitude of each optical candidate, we estimate a probability of association with a given radio source. We show in Sect. 3.3 that this technique leads to a significant contamination by background sources. Using Monte-Carlo simulations, we correct the estimated probabilities for that effect. We address the issue of completeness and reliability in Sect. 3.4, and associate the optical candidates with their infrared IRAC counterparts in Sect. 3.5.

\subsection{Visual inspection and classification}

All the optical images of radio sources have been inspected visually. Where necessary, the Gaussian components of multiple component sources (Tasse et al. 2006, 2007) have been considered separately. This occurred, for example, in the case of two-component radio sources having each component lying close to a bright optical source. In such cases we have renamed the source following the convention used by Tasse et al. (2006, 2007); Table D.1 gives the correspondence between the original name and the new names. The resulting number of sources to be identified is 620 . For all sources ${ }^{3}$ we present the $i$-band images overlaid with the radio contours in Figs. E.1, E.2 and E.3. A tag appearing in Table D.2 indicates the figure in which each source is presented.

Prior to the likelihood ratio calculation presented in Sect. 3.2, a strong subjective a priori was given on the relation between the radio emission position and the position of its optical counterpart, dependent on the radio morphology classification. This allowed estimates of the location of the radio source host galaxy to be derived, as well as the associated errors. This step is described in Appendix A.

\footnotetext{
3 This is irrespective of whether the identified host is saturated or flagged. Six of the identified sources do not appear in Fig. E.1 (J0220.50348, J0220.4-0350, J0226.0-0542, J0225.9-0545, J0229.9-0447 and J0230.0-0440), because the $i$-band image was either totally corrupted or not available at this location. In those cases we have used the $u^{*} g^{\prime} r^{\prime} i^{\prime} z^{\prime}$ band-merged catalog to proceed with the optical identification.
} 

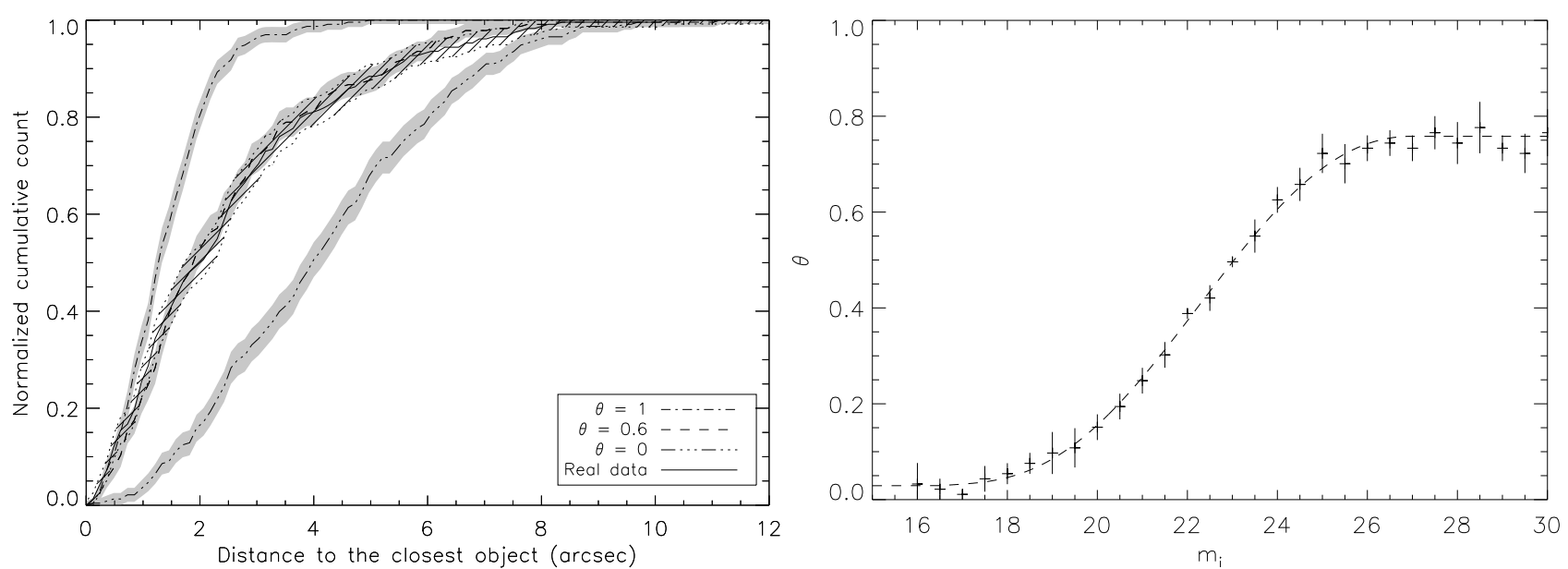

Fig. 2. Left panel: the cumulative distribution of the angular distance from the radio source centroid to the closest neighbour in the optical catalog. In the simulated catalog, the magnitude cut is $m_{i}=24$, and the fraction $\theta(m)$ of radio sources having an optical counterpart is a variable. The first dash-dotted line on the left correspond to $\theta=100 \%$, while dash-dotted line on the right corresponds to $\theta=0 \%$. In this case, the best fit to the distribution of the actual data is obtained with $\theta=60 \%$. The right panel shows the best values of $\theta$ found for different limiting magnitudes. We model $\theta(m)$ as constant above $i=27$ and below $i=17$ and fit by a polynomial between these values (dashed line).

\subsection{Optical identification: the likelihood ratio method}

We quantify the probability that an optical $i$-band candidate is the true optical counterpart of a given radio source by calculating the likelihood ratio as described in Sutherland \& Saunders (1992):

$L R\left(r, m, x_{1}, x_{2} \ldots\right)=\frac{\theta\left(<m, x_{1}, x_{2} \ldots\right) \exp \left(-\frac{r^{2}}{2}\right)}{2 \pi \sigma_{\alpha} \sigma_{\delta} \rho\left(<m, x_{1}, x_{2} \ldots\right)}$

where $m$ is the $i$-band magnitude of the optical candidate, and the values $\left\{x_{1}, x_{2}, \ldots\right\}$ stand for a list of parameters $\left\{X_{1}, X_{2}, \ldots\right\}$ that can be any numerical quantity considered to be relevant, such as the optical colours, or derived photometric redshifts. $\theta\left(m, x_{1}, x_{2} \ldots\right)$ is the a priori probability that a radio source has an observed optical counterpart with magnitude $<m$ and values $\left\{x_{1}, x_{2} \ldots\right\} . \rho\left(m, x_{1}, x_{2} \ldots\right)$ is the surface number density of objects having their magnitude $<m$ and values $\left\{x_{1}, x_{2} \ldots\right\}$. The parameter $r$ is the uncertainty-normalised angular distance between the radio core and the optical host candidate in the band merged $u^{*} g^{\prime} r^{\prime} i^{\prime} z^{\prime}$ optical catalog, defined as $r=\left(\left(\Delta_{\alpha} / \sigma_{\alpha}\right)^{2}+\right.$ $\left.\left(\Delta_{\delta} / \sigma_{\delta}\right)^{2}\right)^{1 / 2}$, where $\Delta$ is the positional difference, $\sigma$ is the uncertainty, and $\alpha$ and $\delta$ are the right ascension and declination respectively. For each $\alpha$ and $\delta$, the uncertainty is the quadratic sum of the uncertainty on the radio position and on the optical position $\sigma_{\alpha}^{2}=\sigma_{\alpha \text {,radio }}^{2}+\sigma_{\alpha \text {,opt }}^{2}$ and $\sigma_{\delta}^{2}=\sigma_{\delta \text {,radio }}^{2}+\sigma_{\delta \text {,opt }}^{2}$. We adopt the T02/T03 astrometry accuracy estimate $\sigma_{\mathrm{opt}} \sim 0.3^{\prime \prime}$, independent of the magnitude $m$. The accuracy of the radio position, $\sigma_{\text {radio }}$, is different for every source; it depends on various parameters such as the local noise level in the radio data, and the Gaussian fitting parameters (Tasse et al. 2006). As explained in Sect. 3.1, we take into account the radio source morphology class to estimate $\sigma_{\text {radio }}$.

Using the formula given by Sutherland \& Saunders (1992), the probability $P_{i \mathrm{~d}}(i)$ of the $i$ th candidate being a true identification is:

$P_{i \mathrm{~d}}(i)=\frac{L R_{i}\left(r, m, x_{1}, x_{2} \ldots\right)}{\sum_{j} L R_{j}\left(r, m, x_{1}, x_{2} \ldots\right)+\left(1-\theta\left(m_{\mathrm{lim}}\right)\right)}$

where $\theta\left(m_{\mathrm{lim}}\right)$ is the fraction of radio sources having a detected optical counterpart at the limiting magnitude of the survey, $i$ refers to the candidate under consideration and $j$ runs over the set of all possible candidates. Contrary to the formulae given by de Ruiter et al. (1977), Benn (1983) and Wolstencroft et al. (1986), this equation includes information from the other candidates, and is self consistent in the sense that $\sum_{i} P_{i \mathrm{~d}}(i) \leq 1$ and $\left\langle\sum_{i} P_{i \mathrm{~d}}(i)\right\rangle=\theta\left(m_{\mathrm{lim}}\right)$ (Sutherland \& Saunders 1992).

We give a first estimate of the probability of association by assuming that $\theta$ and $\rho$ depend only on the object magnitude $m$. In practice, $m$ is taken as the $i$-band magnitude of the optical candidate, and for each radio source we calculate the function $\rho(m)$ in a $2^{\prime}$ square centered on the radio source centroid. This has the advantage of potentially taking into account the effect of clustering, i.e. the variation of the surface density as a function of position. At the limiting magnitude of the survey, $\rho \sim 2 \times 10^{-2} \operatorname{arcsec}^{-2}$. We estimate the values of the function $\theta(m)$ as follows. We consider $i$-band magnitude cuts in the interval $16<i<30$ with an increment $\Delta i=0.5$. For each of these cuts we generate a radio catalog having uniformly distributed positions, and a corresponding optical catalog in which a given fraction $\theta(m)$ of radio sources have an optical counterpart. The optical hosts of radio sources then have their position scattered by the radio and optical positional uncertainties. We then consider the distribution of the angular distance between radio sources and their closest object in the optical catalog. For each value of the limiting magnitude $m$, we compare the distributions of the smallest angular distance for the random catalog and the actual dataset through a Kolmogorov-Smirnov test. The retained fraction $\theta(m)$ is the one corresponding to the maximum Kolmogorov-Smirnov probability (see left panel of Fig. 2 for an example). For each $i$-band magnitude cut, the test is repeated 10 times, so that we can estimate an error bar on $\theta(m)$. The right panel of Fig. 2 shows the variation of $\theta(m)$ with the limiting magnitude. We model $\theta(m)$ using a fit composed of two linear parts and a polynomial of degree 5 . We assume the function $\theta(m)$ we have derived for the Class 1 sources is valid for the extended Class 2 sources as well (see Appendix A).

For each of the 620 radio sources, we have derived the likelihood ratio of the optical counterparts situated in a $20^{\prime \prime}$ radius around the assumed radio centroid. 


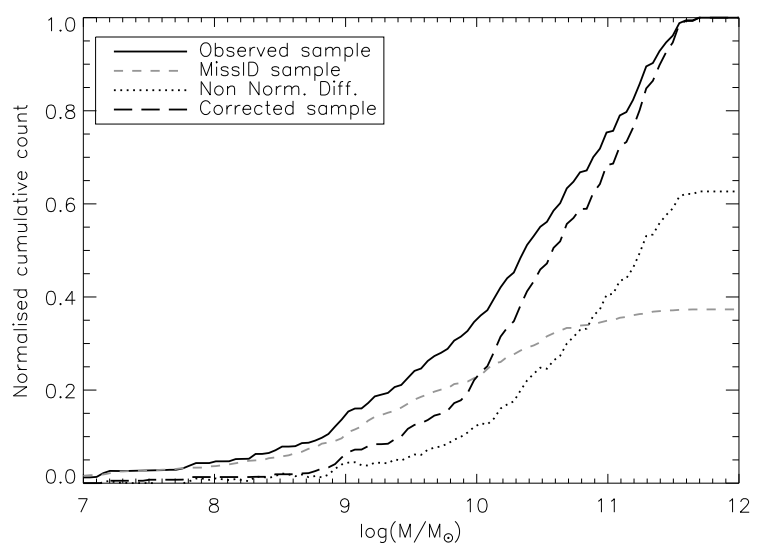

Fig. 3. Stellar mass distributions in the redshift range $0.1<z<1.2$, for the observed radio sources' hosts (solid line) and for the estimated contribution from the misidentifications (grey dashed line). The dotted line ("Non Normalised Difference") corresponds to the difference between these two distributions. We have used such a misidentification contribution subtraction to estimate $\theta(m, M, s S F R, z)$, and give estimates of the association probabilities corrected for the misidentification contribution.

\subsection{Contamination correction}

As with any likelihood matching procedure, random alignment of the radio sources with foreground or background optical galaxies will lead to misidentifications and inaccurate estimates of the association probabilities. In order to derive the probability of association we have only taken into account the $i$-band magnitude of the optical host candidates, and not their $u^{*} g^{\prime} r^{\prime} i^{\prime} z^{\prime}$ magnitude measurements (on which the stellar mass, star formation rate and photometric redshifts estimates depend). Therefore, along dimensions other than the $i$-band magnitude, the distribution of the properties of the radio source optical hosts will be contaminated by these misidentification. Figure 3 (discussed below) shows how the misidentifications contaminate the stellar mass distribution of radio sources' hosts. In order to estimate and correct for this effect, we run Monte-Carlo simulations (as described below) to statistically estimate the dependence of the probability density functions $\theta$ and $\rho$ discussed in Sect. 3.2, on the stellar mass, redshift, and specific star formation rate (that we derive in Sect. 4). We use this knowledge to correct the estimates of the association probability $P_{i \mathrm{~d}}^{i}(j)$.

The parameter space $\left\{z_{\mathrm{ph}}, M, s S F R\right\}$ was first gridded onto a grid in $i$-band optical magnitude $m(15<m<30)$, redshift $\left(0<z_{\text {ph }}<2\right)$, stellar masses $\left(6<\log \left(M / M_{\odot}\right)<13\right)$ and specific star formation rates $\left(-13<\log \left(s S F R / \mathrm{yr}^{-1}\right)<-7\right)$, with steps of $\Delta m=1, \Delta z=0.2, \Delta \log \left(M / M_{\odot}\right)=0.5$, and $\Delta \log \left(s S F R / \mathrm{yr}^{-1}\right)=0.3$ respectively. We estimate the values of the function $\rho$ over the parameter space $\left\{m, z_{\mathrm{ph}}, M, s S F R\right\}$ as follows. For a random subsample of normal galaxies, we calculate the observed number of galaxies in each cell of the parameter space. One obtains $\rho\left(m, z_{\mathrm{ph}}, M, s S F R\right)$ by normalising the total number of sources in such a parameter space to the surface density at the limiting magnitude.

For the radio source host galaxies, in each cell $C$ of the parameter space, the observed number of sources is $n_{\text {obs }}(C)=$ $\sum_{\Omega_{\mathrm{i}, \mathrm{j}}(C)}\left[P_{i \mathrm{~d}}^{i}(j)\right]$, where $P_{i \mathrm{~d}}^{i}(j)$ is the association probability between the $j$ th optical candidate and the $i$ th radio source (Sect. 3.2), and the $\Omega_{i, j}(C)$ is the set of $\{i, j\}$ optical candidates located within the cell $C$. One can write the observed number of radio source hosts $n_{\text {obs }}(C)$ in cell $C$ as $n_{\text {obs }}(C)=n_{\text {true }}(C)+$ $n_{\mathrm{mID}}(C)$, where $n_{\text {true }}(C)$ and $n_{\mathrm{mID}}(C)$ are the observed true and misidentified radio source hosts in the cell. The function $\theta(m, M, s S F R, z)$ is estimated in each cell by removing the misidentified contribution, and by normalising the total number of radio sources in the $\left\{z_{\mathrm{ph}}, M, s S F R\right\}$ parameter space to unity. In order to estimate $n_{\mathrm{mID}}(C)$, we generate 10 simulated radio catalogs in which we associate a fraction $\theta\left(m_{\mathrm{i}}\right)$ of radio sources with galaxies of magnitude $m_{i}$ (see Sect. 3.2), introducing a scatter between the radio and optical positions corresponding to the astrometrical errors of the individual sources in the original radio catalog. We proceed with the optical identification for these simulated catalogs, and derive the association probabilities as described in Sect. 3.2. Knowing the input true optical counterpart, and removing them from our catalog, we can compute the misidentification contribution $n_{\mathrm{mID}}(C)$ (see Fig. 3). We have then re-computed the probabilities of association using these estimates of $\theta$ and $\rho$.

To demonstrate the necessity (and success) of this MonteCarlo correction method, in Fig. 3 we show the probability distribution of the stellar masses of the radio source host galaxies, using the probabilities derived from the previous section. This indicates that $\sim 8 \%$ of radio sources have a host galaxy with a stellar mass in the range $\lesssim 10^{8} M_{\odot}$, which is in stark disagreement with the current belief that radio galaxies ${ }^{4}$ are essentially all hosted by massive galaxies (Best et al. 1998; McLure \& Dunlop 2000; Best et al. 2005). The Monte-Carlo simulations indicate that essentially all of this fraction is associated with contamination from foreground or background sources. Taking properly into account the information on the properties of radio source hosts removes these misidentifications, and produces a much more reasonable stellar mass distribution for radio source hosts. The distributions of other parameters are similarly improved by the Monte Carlo corrections.

\subsection{Completeness and reliability}

In the previous sections, we have associated each radio source with a list of potential optical candidates, and for each one of those we have estimated the probability of it being the true optical counterpart of the given radio source. The approach of many authors to optical identification is to present a list of radio sources, associated with either one or no optical counterpart, with the information on the individual probabilities of association replaced by an averaged reliability level estimated using the formulas given by de Ruiter et al. (1977). However, this approach drives a loss of information about the association probabilities. In order to derive reliable estimates of the stellar mass function of radio sources' hosts, we do not follow this procedure, but rather keep the information on the individual probabilities of association.

In practice, the radio sources' host sample is an extensive list of optical candidates, many of which have low or negligible probability of association with a given radio source. In order to reject the most unlikely optical candidates from the table presented in this paper, we apply a cut on the likelihood ratio $\left(L R_{\text {cut }}=0.1\right)$ to the sample of radio source hosts, after the contamination correction is applied (see Sect. 3.3). Using the probability-weighted number of such rejected sources, we estimate that this rejection drives an incompleteness effect on the level of $\sim 2 \%$. This sample (hereafter S1) is presented in

\footnotetext{
4 Given that the limiting flux density level of the 325 and $610 \mathrm{MHz}$ radio surveys is a few milli-Jansky, we expect that the radio source population probed by our survey is dominated by steep spectrum radio galaxies (Condon 1989; Hopkins et al. 1998; Seymour et al. 2005).
} 
Table D.2. In Sect. 6.3, and in the next paper of the series, we use these full association probabilities in the analysis.

A flag (see last column of Table D.2) allows readers to retrieve a sample of radio sources host galaxies that has the more conventional format discussed above (one or no optical counterpart per radio source; hereafter this is referred to as sample $S 2$ ). To build this sample, for each radio source we consider the optical candidate that has the highest likelihood ratio, provided that it is above the likelihood ratio $1 \mathrm{kmit} L R_{\text {cut }}=0.1$ discussed above. Using the probabilities of association, we estimate that the S2 sample has completeness levels of $\sim 93 \%$ and $~ 95 \%$, and reliability levels of $\sim 86 \%$ and $\sim 85 \%$ for the classes 1 and 2 respectively.

The total number of radio sources having an optical counterpart is $\sum P_{i \mathrm{~d}} \sim 482$. Thus $\sim 76 \%$ of radio sources have a detected optical counterpart. For comparison, in the CENSORS survey, Best et al. (2003) found that $63 \%$ of their brighter radio sample $\left(S_{1.4 \mathrm{GHz}}^{\lim } \sim 7.8 \mathrm{mJy}\right.$ beam $^{-1}$; composed predominantly of radio-loud AGN) have an optical counterpart in $i$-band images reaching $m_{i}^{\lim } \sim 23$. Considering the $i$-band limiting magnitude of our survey is $m_{i}^{\lim } \sim 25$, our fraction $\sim 76 \%$ seems reasonable.

\subsection{Infrared association}

Following Surace et al. (2004), we associate the optical candidates with the infrared objects of the SWIRE DR2 that are closer than $1.5^{\prime \prime}$. This provides flux density measurements at 3.6, $4.5,5.8,8.0$ and $24 \mu \mathrm{m}$ (Sect. 2.4) for the radio source optical hosts identified above. Considering that the source density in the SWIRE DR2 band-merged catalog is $\sim 3.2 \times 10^{4} \mathrm{deg}^{-2}$, and assuming Poisson statistics, the chance of association with a random background source is $\sim 2 \%$. In the case of detection of more than one source within the search radius we have only considered the closest object.

Of the sample of radio source optical counterparts, $\sim 61 \%$ have been associated with an infrared counterpart at 3.6 and $4.5 \mu \mathrm{m}$, and $\sim 33 \%, \sim 27 \%$ and $\sim 18 \%$ at 5.8, 8.0 and $24 \mu \mathrm{m}$ respectively.

\section{Spectral energy distribution fitting}

The photometric redshift method consists of fitting spectral energy distribution (SED) templates to the observed magnitude measurements and their associated error bars using a standard $\chi^{2}$ minimisation. Such galaxy templates can be built from stellar synthesis codes, and physical properties such as age, stellar mass, or star formation rate can be inferred. However, the radioselected galaxy population is dominated by AGN, whose optical emission may dominate over the contribution from the stellar population to the overall SED, such as in the extreme case of an optical quasar. In this section, we use two photometric redshift approaches, the combination of which allows us to: (i) derive physical quantities related to the observed galaxies in our survey; (ii) address the issue of identifying the type-1 AGN; and (iii) assess the reliability of the photometric redshifts.

\subsection{Theoretical approach: ZPEG}

The first SED fitting method uses the $\mathrm{ZPEG}^{5}$ photometric redshift code (Le Borgne \& Rocca-Volmerange 2002). The SED

\footnotetext{
5 The ZPEG code is available online at http://www2.iap.fr/ cgi-bin/pegase/zpeg.pl
}

template library in ZPEG is built from stellar synthesis models with 9 evolutionary scenarios. Effects such as metal enrichment, dust extinction, and nebular emission lines are coherently taken into account depending on evolution scenarios (see Le Borgne \& Rocca-Volmerange 2002, for more details). This approach yields quantitative information on the physics of galaxies. Specifically, ZPEG gives estimates and associated error bars for redshift, stellar mass, and star formation rate. Since the true star formation history of a galaxy can deviate from the idealised scenarios outlined above, the specific star formation rate is averaged over 0.5 Gyr $\left(\mathrm{SFR}_{0.5}\right.$ hereafter, see Sullivan et al. 2006). Dust emission has not been included in these models, and hence this method can only be used in the wavelength range $\lambda \lesssim 1 \mu \mathrm{m}$. The uncertainties associated with these quantities are discussed in more detail in Sect. 6 and by Sullivan et al. (2006). Estimates of the photometric redshift and associated parameters are presented in Table D.2 (see Cols. 18-20).

\subsection{Semi empirical approach}

The second SED fitting method uses semi-empirical SED templates. The SWIRE template library ${ }^{6}$ (Polletta et al. 2007) contains 25 templates including 3 ellipticals, 7 spirals, 6 starbursts, 7 AGN (3 type-1 AGN, 4 type-2 AGN), and 2 composite (starburst+AGN) templates. They are partly based on theoretical SED models, for example the elliptical, spiral and starburst templates (GRASIL code, Silva et al. 1998), and partly on observations, as in the case of the AGN templates, built using data from large surveys such as the SDSS, SWIRE, and FIRST (Becker et al. 1995). For more information on the SWIRE template library see Polletta et al. (2007). This method has the advantage of covering a large wavelength range from far infrared to soft uv light (1000 $\AA$ to $1000 \mu \mathrm{m})$, as well as probing a wide range of objects from normal galaxies to quasars. Using a standard $\chi^{2}$ minimisation procedure with the redshift and the SED normalisation as free parameters, we have fitted the $u^{*} g^{\prime} r^{\prime} i^{\prime} z^{\prime}$ and IRAC flux density measurements. Converting the $\chi^{2}$ in the $\{t, z\}$-space to probability, and normalising to unity, then integrating through the $z$-axis at $t$ we obtain the probability of a template $t$ being the true SED. We will make use of these probability estimates in Sect. 5.1.

Figure 4 shows that the reduced $\chi^{2}$ distribution of the best fit templates is bimodal: $\sim 26 \%$ of the normal galaxy population and $\sim 34 \%$ for the radio sources hosts have a $\chi_{\text {red }}^{2}>100$. This effect can be caused by the underlying true SED of these objects being very different from the set of SEDs present in the SWIRE template library. We have investigated this by comparing the ZPEG output parameter distribution of the $\chi_{\text {red }}^{2}>100$ objects to the rest of the population. The stellar mass, redshift and star formation rate distributions look similar for these two populations, suggesting that the poor $\chi^{2}$ fit for these objects is due to problems with the infrared photometry (e.g. variability that is higher in infrared than in optical): if the objects having $\chi_{\text {red }}^{2}>100$ were instead a population with a different SED-type then one would expect to find biased optical properties, which is not the case. Therefore, in the following, we only include the galaxies having spectral fits with $\chi_{\text {red }}^{2}<100$, assuming this does not drive any selection effect on the observed properties of radio sources. Results from the SED fitting appear in Table D.2 (see Col. 16).

\footnotetext{
6 The SWIRE template library is available online at http://cass. ucsd. edu/SWIRE/mcp/templates/swire_templates.html
} 


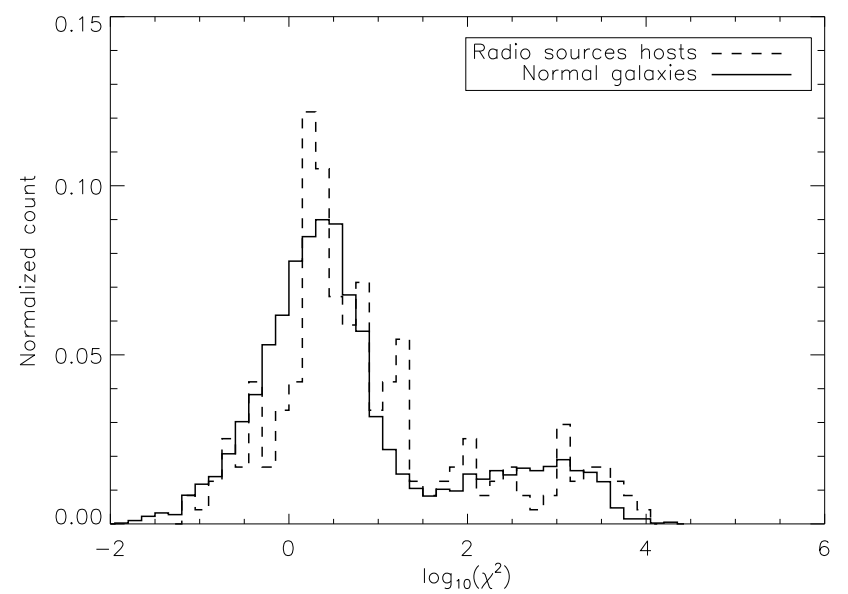

Fig. 4. The reduced $\chi^{2}$ distribution of spectral fits using the SWIRE template library. The secondary bump at $\chi^{2} \gtrsim 100$ shows that $\sim 30 \%$ of the population is not properly fit. Assuming this does not drive any selection effect, we have rejected these sources from the spectral type distribution study.

\section{Classification of radio source hosts}

As discussed in the introduction of this paper, our goal is to study the properties of the host galaxies of radio-loud AGN. In this section, we aim to construct a sample of radio-loud AGN in which the physical parameter estimates, as derived using ZPEG, are reliable. We first identify the radio source hosts for which those estimates can in principle be reliable. This sample satisfies the following conditions:

$1-i<24$

2- $N_{\mathrm{b}} \geq 3$;

3- $i>18$;

4- $\left\{\alpha_{0}, \delta_{0}\right\}$ corresponds to a non-masked area;

5- $0.1<z_{\text {ph }}$ (ZPEG) $<1.2$;

6- $0.1<z_{\mathrm{ph}}(\mathrm{SWIRE})<1.2$;

where $\left\{\alpha_{0}, \delta_{0}\right\}$ is the object position, and $N_{\mathrm{b}}$ is the number of optical bands they are detected in. The selection criterion (1) removes faint objects with large flux density error bars as well as false detections close to bright stars; (2) filters out the objects for which too few flux density measurements are available. The selection criteria (3) and (4) remove the saturated and masked objects respectively, for which the magnitude measurements are corrupted. Since the $4000 \AA$ break of the stellar population is the most constraining feature of SEDs in the optical regime, we restrict our study to the redshift range $z_{\mathrm{ph}}<1.2$, corresponding to the $4000 \AA$ break being in $z^{\prime}$ filter (5-6). Furthermore, we restrict our study to $z>0.1$, since at lower redshifts the large angular sizes of the galaxies may affect the photometry. Below, we classify the objects that satisfy these six criteria as broad-line type1 AGN, narrow-line type-2 AGN, or radio-selected starforming galaxies.

\subsection{Type-1 AGN}

In this section, we pay special attention to identify type-1 AGN whose SED may be dominated by the central core light in the optical domain, in the form of optical and UV continuum emission, or luminous emission lines. These need to be identified since the presence of such objects within the dataset of the radioloud AGN sample introduces a contamination, as their physical
Table 1. The distribution of the original SWIRE template name (Polletta et al. 2007) through our classification (see Sect. 4.2). Table D.2 refers to these templates by refering to the number in brackets.

\begin{tabular}{cccc}
\hline \hline \multicolumn{4}{c}{$\mathrm{N} / \mathrm{T} 2$} \\
\hline Ell13[1] & Sb[6] & I20551[11] & Arp220[16] \\
El12[2] & Sc[7] & I22491[12] & Sey18[17] \\
El15[3] & Sdm[8] & M82[13] & Sey2[18] \\
S0[4] & Sd[9] & N6090[14] & QSO2[25] \\
Sa[5] & Spi4[10] & N6240[15] & \\
\hline \multicolumn{4}{c}{ T1 } \\
I19254[19] Mrk231[20] QSO1[22] & Torus[24] \\
\multicolumn{4}{c}{ BQSO1[21] TQSO1[23] } \\
\hline
\end{tabular}

parameter estimates are unreliable. The results of this classification are encapsuled into a flag in Table D.2 (see Col. 17).

As mentioned in Sect. 2.3, a stellaricity flag has been attached to each source. Since many luminous type-1 AGN are core dominated, unresolved sources in the optical and infrared regimes, the radio source hosts identified as being point-like (see Sect. 2.3 and Table D.2, Col. 10) have been classified as being type-1 AGN. However, the stellaricity parameter cannot be determined for the objects fainter than $i=21$. In order to classify the objects with $i>21$ as type-1 AGN, we have used a combination of criteria based upon (i) the optical colours, and (ii) the goodness of the SWIRE library SED template fits.

The optical colour classification criterion is based on the $g-r$ versus $r-i$ colour-colour diagram (top panel of Fig. 5). Computing the tracks of a type-1 AGN, a starburst, and an elliptical galaxy in that colour-colour space, it is clearly seen that the type-1 AGN occupy a restricted area. This is due to the SED of type- 1 objects being a power law in the optical domain, while the SED of normal galaxies show a high to moderate $4000 \AA$ break. We classify a source as type- 1 if it lies in the region $R\left(g^{\prime}, r^{\prime}, i^{\prime}\right)$ defined as follows:

$$
\begin{aligned}
R\left(g^{\prime}, r^{\prime}, i^{\prime}\right) \equiv & \left(g^{\prime}-r^{\prime}<0.38 \wedge r^{\prime}-i^{\prime}<0.5\right) \\
& \vee\left(g^{\prime}-r^{\prime}>0.38 \wedge r^{\prime}-i^{\prime}<0.2\right)
\end{aligned}
$$

where $\wedge$ and $\vee$ stand for the AND and OR logical connectives.

For the SED-type criteria, we first classified the SWIRE templates in two groups. The first group contains the SEDs in which there is either no contribution, or moderate contribution from an AGN ("N/T2" for Normal/type-2), while the second class contains the templates with strong AGN contribution such as the QSOs ("T1" for type-1). Table 1 shows how the SWIRE templates have been classified in these two categories. We have classified an object as T1 when its probability $P_{T 1}$ (Sect. 4.2) of being a T1-type object satisfies:

$C_{T 1}\left(P_{T 1}\right) \equiv\left(P_{T 1}>60 \%\right)$

$P_{T 1}$ probabilities for the $\mathrm{S} 1$ and $\mathrm{S} 2$ samples appear in Table D.2, Col. 17.

Finally, combining the two selection criteria (Eqs. (3) and (4)) gives: $\left[C_{T 1}\left(P_{T 1}\right)\right] \vee\left[R\left(g^{\prime}, r^{\prime}, i^{\prime}\right)\right]$. Below we investigate the consistency of the selection.

From the study of a large sample of spectroscopically identified sources in the AGN and Galaxy Evolution Survey (AGES, Cool 2006), Stern et al. (2005) have shown that broad line AGN can be well separated from the mean galaxy population in the [3.6]-[4.5] versus [5.8]-[8.0] colour-colour space. In Fig. 5 (bottom panel) we plot the location of radio source hosts in this colour-colour plot. Of the 10 objects classified as T1, $1(10 \%)$ lie outside the area given by Stern et al. (2005); this is in good 

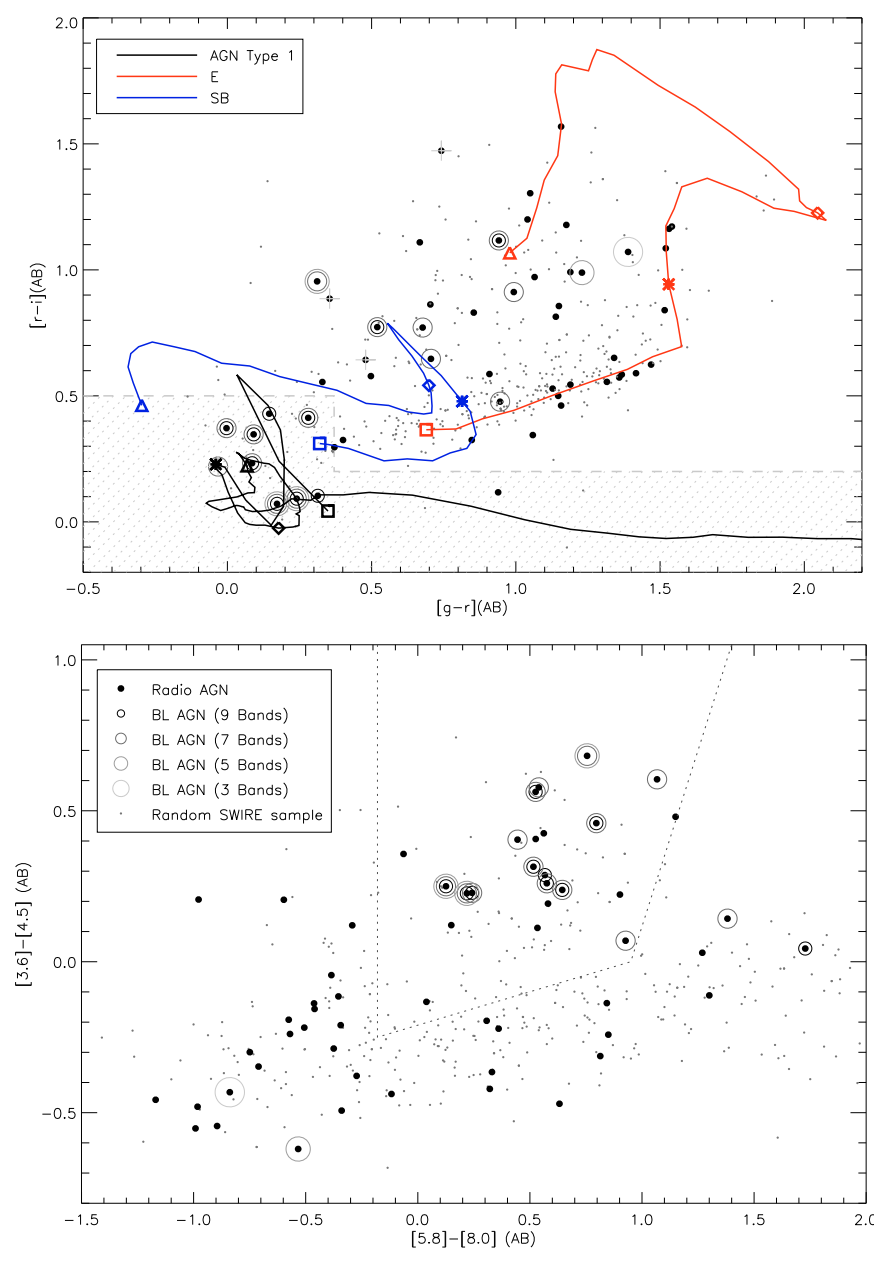

Fig. 5. Top panel: the $g-r$ vs. $r-i$ colour-colour plot for the S2 sample detected in all the $u^{*} g^{\prime} r^{\prime} i^{\prime} z^{\prime}$ and IRAC bands (black dots), and for a subsample of normal galaxies similarly selected (grey dots). We classify as type- 1 those objects which lie in the hashed area. The open circles indicate the classification based on the SED-type criteria (see bottom panel). We plot the colour-colour tracks for a type-1 QSO, an ellipti$\mathrm{cal}$, and a starburst galaxy. The square, star, diamond and triangle symbols stand for redshifts $0,0.5,1.5$, and 2 respectively. Bottom panel: the [3.6]-[4.5] versus [5.8]-[8.0] colour-colour plot for the sources of the same sample. The grey dotted line indicates the region in which Stern et al. (2005) finds $\sim 90 \%$ of the spectroscopically identified broad-line AGN. Based on the SWIRE template library, the objects best fit by a T1 AGN templates are plotted with small black circle. The bigger circles ("BLAGN") indicate the sources classified as T1 AGN when progressively removing the information on the infrared and optical bands ("9 bands": $u^{*} g^{\prime} r^{\prime} i^{\prime} z^{\prime}$ + IRAC available; "7 bands": $u^{*} g^{\prime} r^{\prime} i^{\prime} z^{\prime}+5.8$ and $8.0 \mu \mathrm{m}$ available; "5 bands": $u^{*} g^{\prime} r^{\prime} i^{\prime} z^{\prime}$ available; "3 bands": $g^{\prime} r^{\prime} i^{\prime}$ available, see Sect. 5.1).

agreement with the $9 \%$ fraction estimated by that author to be outside that area. In the $g-r$ vs. $r-i$ colour-colour diagram, the area defined by $R\left(g^{\prime}, r^{\prime}, i^{\prime}\right)$ includes $\sim 5 \%$ of the objects classified as N/T2 using SED fitting, against $80 \%$ of the objects classified as T1 sources. Furthermore, we show in Fig. 7 of Sect. 6 that the ZPEG photometric redshift estimates are in good agreement with the SWIRE template fits for the N/T2, but not for the T1 objects $(\sigma(z) \sim 0.1$ against $\sigma(z) \sim 0.3)$. This coherence suggests that when all IRAC and optical bands are available, we are able to detect the broad line AGN in an efficient way.

However, only $\sim 37 \%$ of our radio source host galaxies are detected in all 9 optical and infrared bands, and it is likely that band availability affects the effectiveness of our SED-type selection technique. In order to address this, we assume that for the sample of sources detected in all 9 bands we have effectively detected all the true type- 1 sources. For this bright sample, we recalculate the photometric redshifts using the SWIRE template library only with their (1) $u^{*} g^{\prime} r^{\prime} i^{\prime} z^{\prime}+5.8$ and $8.0 \mu \mathrm{m}$ (2) $u^{*} g^{\prime} r^{\prime} i^{\prime} z^{\prime}$ and (3) $g^{\prime} r^{\prime} i^{\prime}$ flux density measurements. It can be seen from Fig. 5 that the removal of infrared data has a large influence on the SED-type classification. In the cases where only optical data are available, it appears that our selection criteria leads to $2 / 37 \sim 5.4 \%$ type- 1 AGN not being identified as such, whereas essentially all type-1 AGN are identified when infrared data is available. Since $\sim 39 \%$ of radio sources do not have infrared IRAC measurements, we estimate that the contamination by type- 1 AGN in the sample of radio source host galaxies classified as type- 2 is $\sim 2 \%$.

\subsection{Starburst galaxies}

The intense star formation occurring in starburst galaxies is known to produce a significant amount of radio emission. Since our purpose is to study the triggering processes and the evolution of the radio-loud AGN population there is a need to identify these starburst galaxies in the sample.

Figure 6 shows the relation between the SFR and $1.4 \mathrm{GHz}$ radio power given by Cram (1998), as well as the location of our sources in that plane. We have used the ZPEG star formation rate estimator $\mathrm{SFR}_{0.5}$ and the $1.4 \mathrm{GHz}$ radio power as estimated using the ZPEG photometric redshift and the spectral index (Tasse et al. 2006, 2007). We classify a source as a starburst galaxy if the contribution from star formation to the radio luminosity is higher than $10 \%$, which leads to the selection of 5 radio sources within the subsample selected in Sect. 5. As mentioned in Sect. 4.1, the ZPEG SFR estimate is averaged over $0.5 \mathrm{Gyr}$ while a starburst may occur on a shorter time scale, $\sim 0.1$ Gyr. We investigate this issue by estimating the number of starbursts we should observe within our dataset. To do this we consider the starburst radio luminosity function given by Oliver et al. (1998), combined with the evolution of the starburst luminosity function up to $z=1$, as given by Pozzi et al. (2004). Given our flux density limit at $1.4 \mathrm{GHz}$ of $\sim 1.5 \mathrm{mJy}$, we calculate there should be 6.2 starburst galaxies within our sample. This good correspondence with the number of sources classified as starbursts suggests that the remaining sources are radio-loud AGN. The N/T2/T1 and starburst classification for the S1 and S2 samples is included in the flag reported in the last column of Table D.2.

\section{Accuracy and reliability of derived ZPEG parameters}

\subsection{Photometric redshifts and stellar masses}

As part of the SNLS (SuperNovae Legacy Survey), Sullivan et al. (2006) have discussed the uncertainties in the ZPEG output paramaters in great detail. Their photometric dataset is very similar to ours as they use the broad band CFHTLS-D1 and D4 deep survey data, while we use the CFHTLS-W1. These fields were imaged in the same $u^{*} g^{\prime} r^{\prime} i^{\prime} z^{\prime}$ filters, and although the observations differ in that the Deep surveys are deeper by a factor of $\sim 2$, this should not give rise to any systematic differences between the accuracy of their photometric redshifts and ours.

Using a sample of 116 galaxies having measured spectroscopic redshift, Sullivan et al. (2006) estimate the uncertainties of the ZPEG photometric redshifts and associated parameters. Specifically, the distribution of $\Delta z=z_{\text {spec }}-z_{\text {phot }}$ has a median 


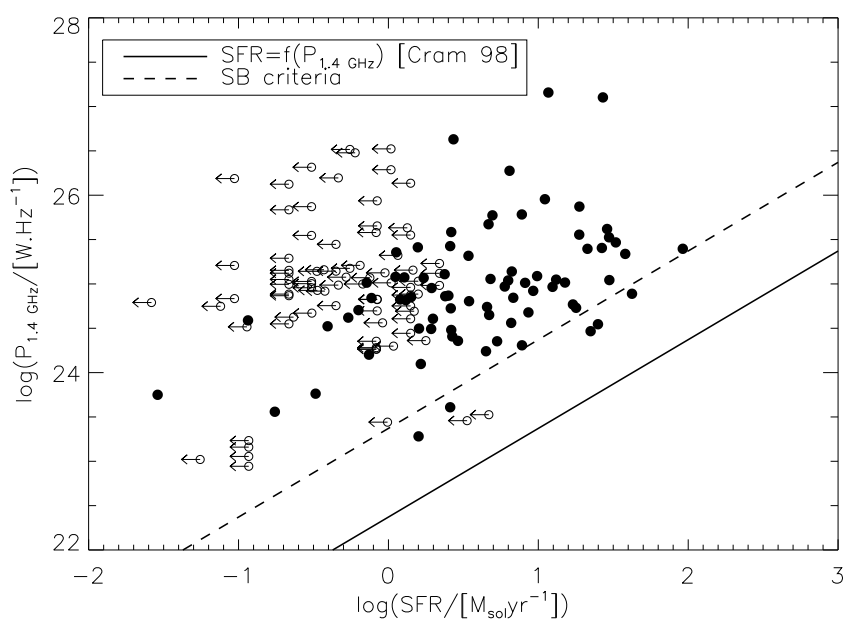

Fig. 6. The distribution of the radio sources in the $P_{1.4}-$ SFR plane. The open circles indicate upper limits. The solid line is the SFR- $\mathrm{P}_{1.4}$ relation given by Cram (1998). Below the dashed line, the contribution by star formation to the radio power is higher than $10 \%$. In order to retrieve a purely radio-loud AGN sample, in the final source list, we have flagged these sources as being starburst-like.

(or $50 \%$ quantile) offset of $q_{0.5}(\Delta z)=0.02$, and a $90 \%$ quantile of $q_{90}(\Delta z)=0.15$. Assuming the distribution to be normal, this corresponds to a standard deviation $\sigma(\Delta z)=0.09$. In order to check that this estimate is compatible with our dataset, we plot the distribution $\Delta z^{\prime}=z_{\mathrm{ZPEG}}-z_{\text {SWIRE }}$ of photometric redshifts as estimated by the ZPEG and SWIRE template libraries respectively for the normal (N/T2) objects detected in at least 7 bands (Fig. 7). Although the ZPEG and SWIRE libraries are built in a very different manner (Sect. 4), for the N/T2 sources the distribution of $\Delta z^{\prime}$ fits a normal distribution with a standard deviation of $\sigma\left(\Delta z^{\prime}\right)=0.13$. This higher value is expected as we compare two independent photometric redshift estimates, corresponding to a difference of a factor of $\sqrt{2}$ between $\sigma(\Delta z)$ and $\sigma\left(\Delta z^{\prime}\right)$, in agreement with the factor $\sim 1.4$ observed. For comparison, we also show the results for the object classified as type-1 AGN (T1), whose ZPEG photometric redshifts are likely to be erroneous due to AGN light: as expected, the two photometric redshift estimates do not agree well for these sources.

Sullivan et al. (2006) also discussed in detail the accuracies of the stellar mass estimates. In the most extreme case of galaxies experiencing recent star formation events, they derive $q_{50}\left(\Delta \log \left(M /\left[M_{\odot}\right]\right)\right) \sim-0.02, q_{90}\left(\Delta \log \left(M /\left[M_{\odot}\right]\right)\right) \sim 0.37$. Furthermore, Sullivan et al. (2006) found that the error bars as estimated by ZPEG based on the $\chi^{2}$ statistics are consistent with the statistics of the observed errors $\left(z_{\mathrm{spec}}-z_{\text {phot }}\right)$, suggesting that the uncertainties for the individual objects are properly estimated.

Using the stellar masses and photometric redshifts estimates, below we will show that the stellar mass function and radio luminosity function derived using our dataset are consistent with previous results.

\subsection{The influence of emission lines}

The activity in the central core of powerful radio-loud AGN is known to produce luminous emission lines with $L_{[\mathrm{OII}]} \sim 10^{40}-$ $10^{44} \mathrm{erg} \mathrm{s}^{-1}$ (e.g. McCarthy 1993; Zirbel \& Baum 1995).

In order to investigate whether these emission lines can influence the photometric redshift and the stellar masses estimates, we generated a catalog of galaxies with SED taken randomly

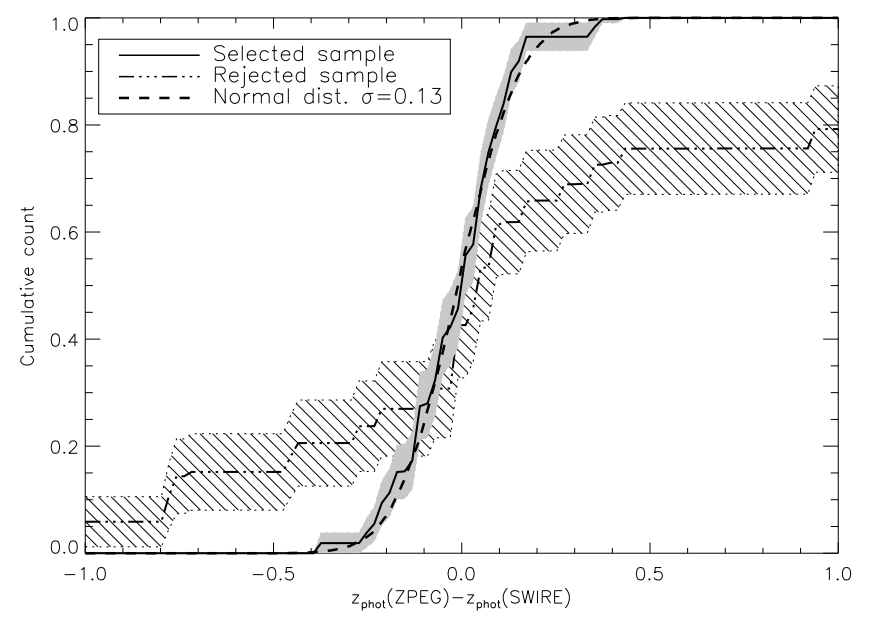

Fig. 7. For a sub-sample of sources detected at 3.6 and $4.5 \mu \mathrm{m}$, we can compare the photometric redshifts as estimated using the ZPEG and the SWIRE template libraries. Here we show the cumulative distribution of $z_{\text {ZPEG }}-z_{\text {SWIRE }}$ for the T1 sources ("rejected") and for the N/T2 sources ("selected"). The N/T2 sample is best fitted by a normal distribution with sigma $=0.13$.

Table 2. Influence of the presence of emission lines at the level $L[\mathrm{OII}]$ on the estimates of photometric redshifts and stellar masses. $q_{0.5}$ refers to the median value, while $q_{0.9}$ refers to the $90 \%$ quantile.

\begin{tabular}{ccccc}
\hline \hline$L[\mathrm{OII}]$ & \multicolumn{2}{c}{$\Delta z$} & \multicolumn{2}{c}{$\Delta \log \left(M / M_{\odot}\right)$} \\
{$\left[\mathrm{erg} \mathrm{s}^{-1}\right]$} & $q_{0.5}$ & $q_{0.9}-q_{0.5}$ & $q_{0.5}$ & $q_{0.9}-q_{0.5}$ \\
\hline$<38$ & -0.003 & 0.09 & -0.03 & 0.45 \\
40 & -0.004 & 0.09 & -0.04 & 0.45 \\
42 & 0.008 & 0.20 & -0.04 & 0.76 \\
44 & -0.027 & 0.34 & -0.68 & 0.64 \\
\hline
\end{tabular}

from the ZPEG SED library, corresponding to random stellar mass, age, star formation rate, and redshift. To each SED, we added emission lines: the [OII] line luminosities were varied between $10^{38}$ and $10^{44} \mathrm{erg} \mathrm{s}^{-1}$, while the other emission line luminosities were scaled from the [OII] luminosity using the emission line luminosity ratios given by McCarthy (1993). We generated the corresponding $u^{*} g^{\prime} r^{\prime} i^{\prime} z^{\prime}$ magnitudes, and estimated the photometric redshifts using ZPEG. Table 2 shows the results of this analysis. At $L_{[\mathrm{OII}]}<10^{40} \mathrm{erg} \mathrm{s}^{-1}$, the influence of emission lines is negligible: although there are a few outliers, the statistics are comparable to the case of no emission lines $\left(L_{[\mathrm{OII}]}<10^{38} \mathrm{erg} \mathrm{s}^{-1}\right)$ for both photometric redshifts and stellar masses. On the other hand, for $L_{[\mathrm{OII}]} \geq 10^{42} \mathrm{erg} \mathrm{s}^{-1}$ systematics and outliers become increasingly important. Using the photometric redshifts estimates of ZPEG, we estimate the radio power range of the sources in our selected sample (Sect. 5) to be $\log _{10}\left(P_{1.4 \mathrm{GHz}} / \mathrm{W} . \mathrm{Hz}^{-1}\right) \lesssim 26$ for $\sim 90 \%$ of the sources. From the [OII] line luminosity - radio power relation (McCarthy 1993; Best et al. 2005), this radio power corresponds to $L_{[\mathrm{OII}]} \lesssim 10^{40}$ $10^{41} \mathrm{erg} \mathrm{s}^{-1}$. We therefore conclude that within the radio power range probed by our survey, the presence of emission lines should not significantly affect the photometric redshift estimates.

\subsection{Consistency tests}

In this section we carry out tests to check the consistency of the identification, the type-2 AGN selection technique, and the ZPEG output parameter estimates. 


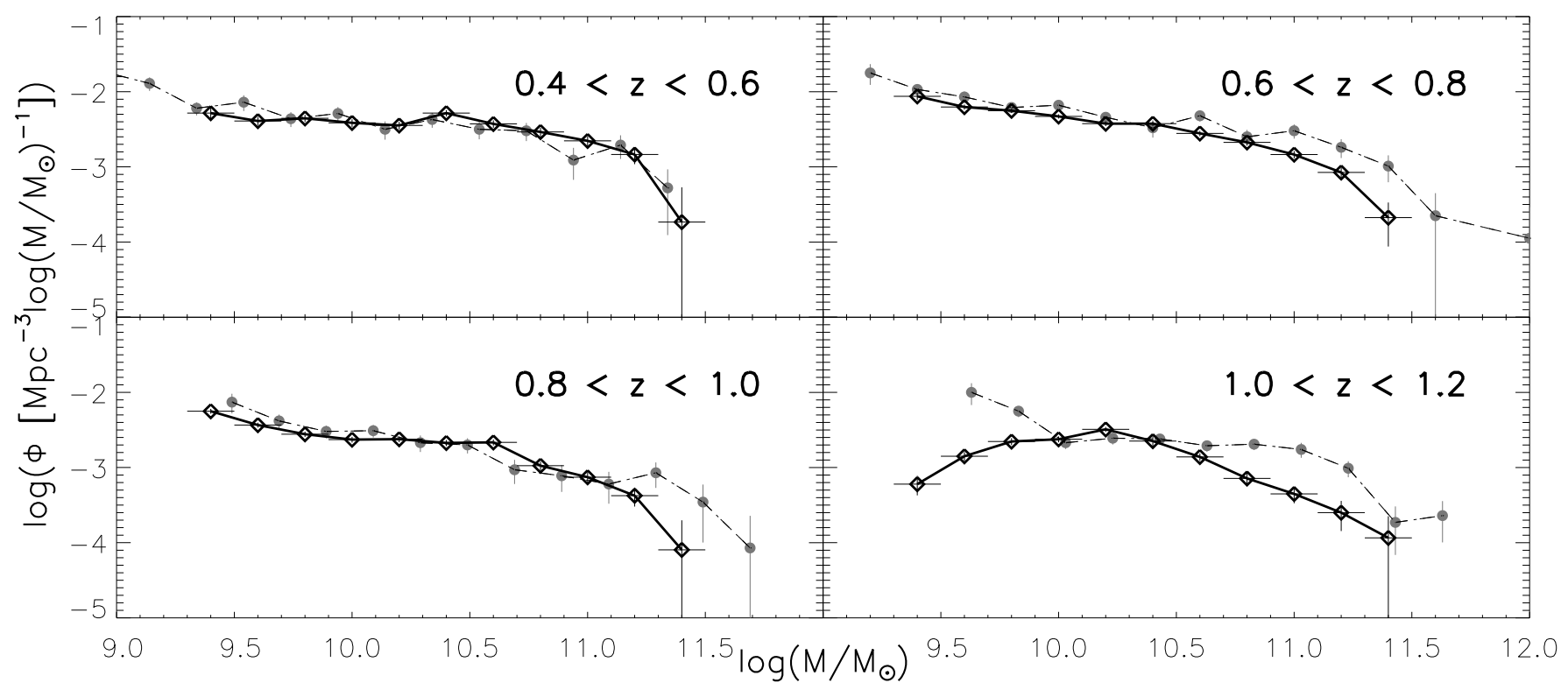

Fig. 8. In order to test the consistency of our results, we have derived the stellar mass function for normal galaxies (open diamonds and thick solid line), by using the $1 / V_{\max }$ comoving number density estimator. Our estimates are in good agreement with measurements in the GOODS surveys (Fontana et al. 2006, grey dots and dash-dot line), which suggests that the stellar mass estimates are reliable for normal galaxies.

We first derive the stellar mass function for normal galaxies $\left(\phi_{\mathrm{Opt}}\right)$ by using the $1 / V_{\max }$ estimator (Schmidt 1968), which corrects for the fact that our sample is magnitude limited. This procedure is described in detail in Appendix C.1. Figure 8 shows the stellar mass function of all galaxies in the redshift ranges: $0.4<z<0.6,0.6<z<0.8,0.8<z<1.0$, and $1.0<z<1.2$. A number of authors have estimated the galaxy mass function using different techniques, in various redshift ranges (see Fontana et al. 2006, for a general review). We compare our estimates of $\phi_{\text {Opt }}$ to the stellar mass function as estimated by Fontana et al. (2006) in the redshift intervals $0.4<z<0.6,0.6<z<0.8$, $0.8<z<1.0$, and $1.0<z<1.3$. These estimates were derived using the GOODS-MUSIC catalogs (Grazian et al. 2006), which contain broad band photometry from the optical to infrared regime, as well as spectroscopic data for $\sim 27 \%$ of the sample. At $z<1$, our estimates of $\phi_{\text {Opt }}$ (see open diamonds and thick solid line in Fig. 8) show good agreement with Fontana et al. (2006, see grey dots and dash-dot line in Fig. 8), for both normalisation and shape. However, in the higher redshift bin, the differences between the two estimates can be as high as $\sim 0.6$ dex. These differences may be due to the difficulty of deriving reliable stellar mass estimates at $z \gtrsim 1$, in the absence of infrared data. The low comoving number density of low stellar mass galaxies in the higher redshift bin are due to an incompleteness effect discussed in detail in Fontana et al. (2004).

We have also computed the radio luminosity function (RLF) of the radio-loud AGN in our sample, by using the comoving number density estimator described in Appendix C.2. The RLF of radio sources in our sample (see open diamonds in Fig. 9) is in good agreement with the Willott et al. (2001) RLF estimates derived from the 7CRS, 3CRR, and 6CE radio source samples selected at $150 \mathrm{MHz}$ (see dashed line in Fig. 9).

These results provide confidence in the photometric redshift and stellar mass measurements (at least to $z \sim 1$ ) for both the normal galaxies and the radio source hosts.

\section{Summary}

We have identified 620 low frequency radio sources (Tasse et al. 2006) overlapping with the CFHTLS-W1 field (T02/T03

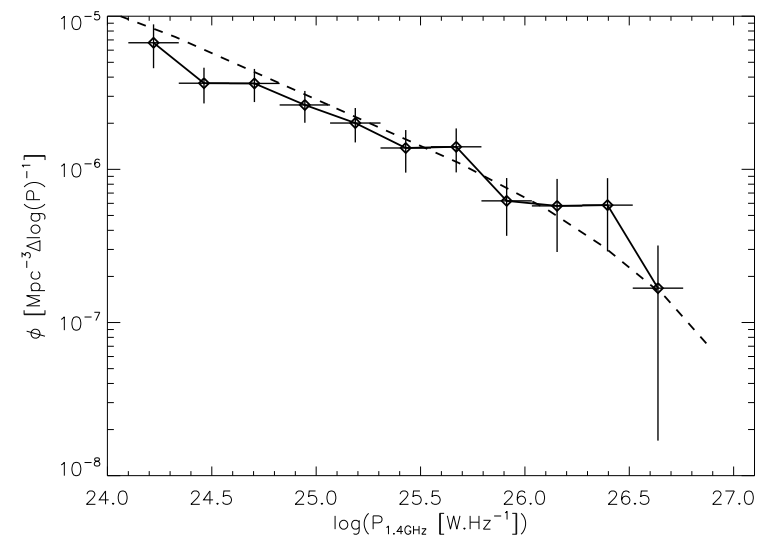

Fig. 9. We compare our estimate of the radio luminosity function in $0.1<z<1.2$ (open diamonds) with the Willott et al. (2001) RLF estimate (dashed line). Both the normalisation and slope are in good agreement, confirming that the photometric redshifts of the sample of $\mathrm{N} / \mathrm{T} 2$ radio-loud galaxies are reliable.

release). A proper use of the likelihood ratio method (Sutherland \& Saunders 1992) allowed us to derive estimates for the probability of association with a given optical candidate. About $75 \%$ of the radio sources have been associated with an optical counterpart. This result is consistent with both our Monte-Carlo estimate and previous results from other surveys. Using the infrared SWIRE survey catalogs, we have associated the radio sources with their optical and infrared counterparts.

In order to have a good overall understanding of the identified sample, we have fitted SED templates to the flux density measurements using two very different approaches: the first is theoretical and gives quantitative information, while the second is empirical and gives qualitative insights. In order to obtain a sample with reliable estimates of physical parameters, we classify the radio source host galaxies as type-1, type- 2 or starbursts.

We have carried out preliminary analyses that suggest that (i) the identification procedure has been conducted properly, (ii) the estimates of the stellar mass of normal galaxies are 
reliable and (iii) the photometric redshifts of the sample of type2 radio source host galaxies are reliable. In the next paper of the series, we will give further evidence that the physical parameter estimates are reliable for the selected sample, and we will present and discuss the properties and environment of radio-loud AGN.

Acknowledgements. The authors have made use of the NASA/IPAC Extragalactic Database (NED), which is operated by the Jet Propulsion Laboratory, Caltech, under contract with the National Aeronautics and Space administration. The optical images were obtained with MegaPrime/MegaCam, a joint project of CFHT and CEA/DAPNIA, at the CFHT which is operated by the National Research Council (NRC) of Canada, the Institut National des Sciences de l'Univers of the Centre National de la Recherche Scientifique (CNRS) of France and the University of Hawaii. This work is based on data products produced at TERAPIX and at the Canadian Astronomy Data Centre as part of the CFHTLS, a collaborative project of NRC and CNRS. The authors thank the referee for a number of helpful suggestions, and Herve Aussel for useful discussions on the Terapix data release.

\section{Appendix A: Radio source classification}

In order to identify the host galaxies of radio sources, an a priori has to be put on the true location of the host galaxy with respect to radio source.

- Class 1: sources are classified as Class 1 when the radio emission is assumed to be produced at the physical location of optical emission (detected or not). This occurs in sources such as starbursts, compact core dominated radio sources or radio sources where the radio core can be clearly identified. In these cases, knowing the errors on the radio and optical positions, a statistical approach can be used directly to identify the optical progenitor of a considered radio source at a given position (Sect. 3.2).

- Class 2: when no radio core is identified (as often happens) in classical double lobe FRII (Fanaroff \& Riley 1974) radio sources, only a weak a priori can be considered for the optical host position. Following Best et al. (2003) we have used a case-by-case approach: when the morphology does not give any clue to the location of the optical host, we classify the radio source as Class 2 . The position of the host and associated errors are estimated based on the location of the multiple Gaussian fitting components. This aspect is discussed in greater detail in Best et al. (2003).

- Class 3: when the environment has a large effect on the radio morphology, the flux weighted radio centroid and associated error bars can be very far from the real optical host. When suggested by the combination of radio and optical properties (such as an elongated lobe pointing to a bright optical object), we use the radio morphology to determine the position of the optical counterpart and we classify the object as Class 3. Note that, because of that case-to-case approach, we cannot calculate the completeness and reliability level of the Class 3 subsample as we do for Class 1 and 2 objects (Sect. 3.4). Comments on the Class 3 individual sources are given in Appendix B.1.

- Class 4: we have classified as Class 4 the resolved radio sources for which the morphology does not suggest the presence of jets. Radio halos and relics are a part of this class. Comments on these sources can be found in Appendix B.2.

- Class 5: when the radio source overlaps a bright saturated source, or a satellite track for example, or when a radio source overlaps a masked object (a bright saturated object or a satellite track), we have classified the source as Class 5, meaning that we cannot proceed with the identification.

\section{Appendix B: Comments on individual sources}

In this section, we give comments on the radio sources classified as Class 3 and Class 4 sources (see Sect. 3.1 for the class definitions).

\section{B.1. Class 3 sources}

J0216.6-0527 (Fig. E.2): Although only the eastern lobe was detected by Tasse et al. (2007), a $3 \sigma$ level component is clearly elongated along an east-west axis, suggesting the optical host to be an $m_{r}=19.2$ galaxy.

J0217.6-0513 (Fig. E.1): Only the east side $5 \sigma$ lobe appears in the Tasse et al. (2006) source list, but radio contours at $3 \sigma$ suggest the source is extended in the east-west direction. The uncataloged west-side lobe corresponds to a $m_{r}=20.51 \mathrm{object}$ that we chose as the optical identification.

J0217.7-0541 (Fig. E.1): The radio source appears to be a small head-tail radio galaxy. The weighted radio position is $\sim 6-8^{\prime \prime}$ off the bright $m_{i} \sim 21$ galaxy that lies within the two radio lobes. We have identified this object as being the progenitor.

J0218.9-0401 (Fig. E.1): Only the eastern lobe appears in the Tasse et al. (2006) $325 \mathrm{MHz}$ catalog, although there appears to be a second lobe at the $4 \sigma$ level. We have chosen the $m_{r}=24.21$ object lying at the center to be the optical counterpart.

J0219.1-0357 (Fig. E.1): The asymmetry angle of this source is obviously negligible, but the luminosity of the two radio lobes is quite different. This shifts the radio source centroid towards the south-east of the region. We choose the faint $m_{i} \sim 25.02$ source to be the optical host.

J0219.5-0507 (Fig. E.2): As for J0216.6-0527, only one of the two lobes is detected by Tasse et al. (2007). Two objects might be considered to be possible optical candidates: the one overlapping the lobe on the north-east and the $m_{r}=21.1$ object lying in the middle of the two lobes. Since the radio morphology is likely an FRII, we have considered this later object to be the host.

J0219.9-0518 (Fig. E.1): For this double lobe radio source, the flux weighted radio position is too far from the obvious very bright $m_{i} \sim 16$ host galaxy.

J0220.6-0517 (Fig. E.1): This radio source appears to be a double lobe with the second undetected on the east side of the image. The detected lobe points to an $m_{r} \sim 22$ galaxy that we have considered to be the optical counterpart.

J0223.4-0427 (Fig. E.1): As for J0217.7-0541 the flux weighted central position is well away from the obvious $m_{i}=$ 20.6 optical counterpart.

J0224.2-0528 (Fig. E.1): Although the identification is obvious, the flux weighted centroid does not lead to the selection of that candidate. We have chosen the bright $\mathrm{m}_{r}=20.8$ galaxy to be the optical host.

J0225.5-0524 (Fig. E.1): Only the western radio component appears in the radio catalog, the eastern lobe being too faint. We consider the $m_{r}=22.51$ object at the center to be the optical counterpart.

J0227.1-0543 (Fig. E.1): This elongated east-west lobe points to a $m_{r}=22.58$ object that we have considered to be the progenitor.

J0228.0-0400 (Fig. E.1): This source, elongated along the north-south axis, has only one of its component cataloged. We 
have considered the bright object lying at the center to be the optical counterpart.

J0228.2-0503 (Fig. E.1): Similar to the case of J0219.1-0357 the asymmetry of the flux inside the two radio lobes makes the flux weighted centroid shift towards the north, whereas the optical host is obviously the bright candidate lying at the center.

\section{B.2. Class 4 sources}

J0217.0-0449 (Fig. E.3, bottom panel): This object is one of the most extreme radio sources in the combined radio sample. Its angular size is almost $2^{\prime}$ and its spectral index is one of the steepest in the sample with $\alpha_{1400}^{325}=-1.65$. The two lobes are separated by almost $1^{\prime}$ and no bright optical counterpart is present in that area. The most likely candidate has an $r$-band magnitude $m_{r}=24.5$ and a photometric redshift estimate of $z_{\mathrm{p}}=0.88_{-0.08}^{+0.04}$, which would make the physical diameter at the radio source location $D_{\mathrm{p}} \sim 0.92 \mathrm{Mpc}$. We suggest that this radio source might correspond to the latest stage of the evolution of a radio source, when no more high energy electrons are injected into the IGM.

J0219.7-0400 (Fig. E.3, top panel): This object has been reported to be a promising radio halo or radio relic candidate in Tasse et al. (2006). The overlay with the $i$-band imaging shows that the diffuse radio emission corresponds to an overdensity of optical galaxies.

\section{Appendix C: Number density estimator}

\section{C.1. Normal galaxies}

In order to compute number densities in the comoving space, for the normal galaxies we use the standard $1 / V_{\max }$ estimator first described in Schmidt (1968):

$\left\langle\phi_{R}\right\rangle=C \sum_{\Omega_{i}(R)}\left[1 / V_{\max }^{i}\right]_{R}$

where $V_{\max }$ is the maximum comoving volume over which a given galaxy can enter our sample, $C$ is a constant designed to compensate for the random selection of the objects in the optical catalog and the $\sim 30 \%$ of the optical field that is masked, and $\Omega_{i}(R)$ is the set of galaxies that lie in each region $R$ of the parameter space. For example, in computing the stellar mass function, the regions $R$ correspond to stellar mass bins. The error bar associated with $\left\langle\phi_{R}\right\rangle$ is:

$\sigma\left(\left\langle\phi_{R}\right\rangle\right)=C \sqrt{\sum_{\Omega_{i}(R)}\left[1 / V_{\max }^{i}\right]_{R}^{2}}$.

In practice, we set $V_{\max }=V\left(z_{\max }\right)-V\left(z_{\min }\right)$ where $V(z)$ is the comoving volume enclosed out to a given $z$, while $z_{\max }$ and $z_{\min }$ are the maximum and minimum redshifts for which a given object is selected. Since the CFHTLS optical data is homogeneous in depth, to calculate comoving volumes we assume that the solid angle of the survey area is not magnitude-dependent. The lower and upper redshift bounds $z_{\min }$ and $z_{\max }$ depend upon (i) the redshift range corresponding to the cell $R$ of the parameter space in which we estimate $\left\langle\phi_{R}\right\rangle$ (ii) the selection criteria of our optical data (Sect. 5). Estimating $z_{\min }$ and $z_{\max }$ for the selection criterion (i) is trivial and depends on the redshift bin used to derive the comoving number density. Estimating $z_{\min }$ and $z_{\max }$ based on the selection criterion (ii) uses the magnitude selection criteria of Sect. 5. For each optical object we consider the best fitting ZPEG SED template. The lower bound $z_{\min }$ is then the redshift corresponding to an observed $i$-band magnitude $i=18$, whereas $z_{\max }$ is either the redshift for which $i=24$ or the maximum redshift for which the given object is detected in three bands. For each object, the final $z_{\min }$ and $z_{\max }$ to be used is derived as $z_{\min }=\max \left(\left\{z_{\min }(\mathrm{i}), z_{\min }(\mathrm{ii})\right\}\right)$ and $z_{\max }=\min \left(\left\{z_{\max }(\mathrm{i}), z_{\max }(\mathrm{ii})\right\}\right)$ where the indices (i) and (ii) refer the selection criteria (i) and (ii) defined above.

\section{C.2. Radio source hosts}

The S1 sample presented in Sect. 5 contains, for each radio source $i$, an association probability $P_{i \mathrm{~d}}^{i}(j)$ that its true counterpart is the $j$ th optical candidate. Using the $\mathrm{S} 1$ sample we estimate the mean comoving number density in a region $R$ of the parameter space as:

$\left\langle\phi_{R}\right\rangle=C \sum_{\Omega_{i, j}(R)}\left[P_{i \mathrm{~d}}^{i}(j) / V_{\max }^{i}(j)\right]_{R}$

where $\Omega_{i, j}(R)$ is the set of $\{i, j\}$ optical candidates which are located within the region $R, V_{\max }$ is the maximum comoving volume over which a given radio source can be selected and $C$ is a constant discussed above. The uncertainties associated with the comoving number density are then calculated as:

$\sigma\left(\left\langle\phi_{R}\right\rangle\right)=C \sqrt{\sum_{\Omega_{i}(R)}\left[P_{i d}^{i}(j) / V_{\max }^{i}(j)\right]_{R}^{2}}$.

Since the radio survey is inhomogeneous in depth, the effective area surveyed depends on the flux density of a given radio source. We have estimated that dependence by comparing our observed source counts to a field with deeper radio source counts (Seymour et al. 2005). In addition to the selection criteria (i) and (ii) discussed above, the upper redshift bound $z_{\max }$ depends upon (iii) the radio power of the given radio source. To calculate this, we consider its radio power and the estimate of the spectral index as derived by Tasse et al. (2006) and Tasse et al. (2007). We estimate $z_{\max }$ as the redshift corresponding to that object being detected at the limiting flux density of the radio survey at its location in either the 325 or the $610 \mathrm{MHz}$ radio image. We finally calculate $z_{\max }$ as $z_{\max }=\min \left(\left\{z_{\max }(\mathrm{i}), z_{\max }(\mathrm{ii}), z_{\max }(\mathrm{iii})\right\}\right)$.

\section{References}

Becker, R. H., White, R. L., \& Helfand, D. J. 1995, ApJ, 450, 559 Benn, C. R. 1983, The Observatory, 103, 150 Bertin, E., \& Arnouts, S. 1996, A\&AS, 117, 393

Best, P. N., Longair, M. S., \& Roettgering, H. J. A. 1998, MNRAS, 295, 549 Best, P. N., Arts, J. N., Röttgering, H. J. A., et al. 2003, MNRAS, 346, 627 Best, P. N., Kauffmann, G., Heckman, T. M., et al. 2005, MNRAS, 362, 25 Cohen, A. S., Röttgering, H. J. A., Kassim, N. E., et al. 2003, ApJ, 591, 640 Condon, J. J. 1989, ApJ, 338, 13

Cool, R. J. 2006, in Am. Astron. Soc. Meet. Abstr., 209, 193.06

Cram, L. E. 1998, ApJ, 506, L85

de Ruiter, H. R., Arp, H. C., \& Willis, A. G. 1977, A\&AS, 28, 211

Evans, D. A., Worrall, D. M., Hardcastle, M. J., Kraft, R. P., \& Birkinshaw, M. 2006, ApJ, 642, 96

Fanaroff, B. L., \& Riley, J. M. 1974, MNRAS, 167, 31P

Fontana, A., Pozzetti, L., Donnarumma, I., et al. 2004, A\&A, 424, 23

Fontana, A., Salimbeni, S., Grazian, A., et al. 2006, A\&A, 459, 745

Grazian, A., Fontana, A., de Santis, C., et al. 2006, A\&A, 449, 951

Hardcastle, M. J., Evans, D. A., \& Croston, J. H. 2006, MNRAS, 370, 1893

Hardcastle, M. J., Evans, D. A., \& Croston, J. H. 2007, MNRAS, 376, 1849

Heckman, T. M., \& Kauffmann, G. 2006, New Astron. Rev., 50, 677

Heckman, T. M., Kauffmann, G., Brinchmann, J., et al. 2004, ApJ, 613, 109

Hine, R. G., \& Longair, M. S. 1979, MNRAS, 188, 111

Hopkins, A. M., Mobasher, B., Cram, L., \& Rowan-Robinson, M. 1998, MNRAS, 296, 839 
Jackson, N., \& Rawlings, S. 1997, MNRAS, 286, 241

Kauffmann, G., Heckman, T. M., Tremonti, C., et al. 2003, MNRAS, 346, 1055

Kron, R. G. 1980, ApJS, 43, 305

Laing, R. A., Jenkins, C. R., Wall, J. V., \& Unger, S. W. 1994, in The Physics of Active Galaxies, ed. G. V. Bicknell, M. A. Dopita, \& P. J. Quinn, ASP Conf. Ser., 54, 201

Le Borgne, D., \& Rocca-Volmerange, B. 2002, A\&A, 386, 446

Lonsdale, C. J., Smith, H. E., Rowan-Robinson, M., et al. 2003, PASP, 115, 897

Mathews, W. G., \& Brighenti, F. 2003, ARA\&A, 41, 191

McCarthy, P. J. 1993, ARA\&A, 31, 639

McLure, R. J., \& Dunlop, J. S. 2000, MNRAS, 317, 249

Ogle, P., Whysong, D., \& Antonucci, R. 2006, ApJ, 647, 161

Oke, J. B., \& Gunn, J. E. 1983, ApJ, 266, 713

Oliver, S., Gruppioni, C., \& Serjeant, S. 1998, ArXiv Astrophysics e-prints

Pierre, M., Valtchanov, I., Altieri, B., et al. 2004, J. Cosmology Astro-Particle Phys., 9, 11

Polletta, M., Tajer, M., Maraschi, L., et al. 2007, ArXiv Astrophysics e-prints

Pozzi, F., Gruppioni, C., Oliver, S., et al. 2004, ApJ, 609, 122

Prestage, R. M., \& Peacock, J. A. 1983, MNRAS, 204, 355
Richter, G. A. 1975, Astron. Nachr., 296, 65

Rowan-Robinson, M., Babbedge, T., Surace, J., et al. 2005, AJ, 129, 1183

Schmidt, M. 1968, ApJ, 151, 393

Seymour, N., McHardy, I., Gunn, K., \& Moss, D. 2005, in ESA SP, ed. A. Wilson, 323, 577

Silva, L., Granato, G. L., Bressan, A., \& Danese, L. 1998, ApJ, 509, 103

Stern, D., Eisenhardt, P., Gorjian, V., et al. 2005, ApJ, 631, 163

Sullivan, M., Howell, D. A., Perrett, K., et al. 2006, AJ, 131, 960

Surace, J. A., Shupe, D. L., Fang, F., et al. 2004, VizieR Online Data Catalog, 2255,0

Sutherland, W., \& Saunders, W. 1992, MNRAS, 259, 413

Tasse, C., Cohen, A. S., Röttgering, H. J. A., et al. 2006, A\&A, 456, 791

Tasse, C., Röttgering, H. J. A., Best, P. N., et al. 2007, A\&A, 471, 1105

Tasse, C., Best, P. N., Röttgering, H., \& Le Borgne, D. 2008, A\&A, 490, 893

Whysong, D., \& Antonucci, R. 2004, ApJ, 602, 116

Willott, C. J., Rawlings, S., Blundell, K. M., Lacy, M., \& Eales, S. A. 2001, MNRAS, 322, 536

Wolstencroft, R. D., Savage, A., Clowes, R. G., et al. 1986, MNRAS, 223, 279

Zirbel, E. L., \& Baum, S. A. 1995, ApJ, 448, 521 
C. Tasse et al.: The optical and infrared properties of low-frequency radio sources in the XMM-LSS field, Online Material p 1

\section{Appendix D: Tables}

Table D.1. The 15 objects for which the Gaussian fitting componants where split and renamed. Positions in Cols. 3 and 4 are the flux density weighted ones.

\begin{tabular}{cccc}
\hline \hline Original name & New name & RA & Dec \\
\hline J0215.0-0458 & J0215.0-0458 & 021459.04 & -045811.32 \\
& J0215.0-0457 & 021458.92 & -045741.24 \\
J0216.5-0447 & J0216.6-0447a & 021635.06 & -044701.11 \\
& J0216.6-0447b & 021637.30 & -044736.35 \\
& J0216.5-0447 & 021632.23 & -044741.59 \\
J0218.0-0346 & J0218.0-0346a & 021757.03 & -034602.64 \\
& J0218.0-0346b & 021757.51 & -034648.95 \\
J0219.4-0404 & J0219.4-0404 & 021925.75 & -040418.15 \\
& J0219.4-0403 & 021924.98 & -040352.49 \\
& J0219.5-0403 & 021927.49 & -040344.90 \\
J0219.5-0539 & J0219.5-0539 & 021928.22 & -053948.21 \\
& J0219.4-0539 & 021925.20 & -053911.99 \\
J0220.8-0510 & J0220.8-0510 & 022050.67 & -051017.85 \\
& J0220.8-0509 & 022050.98 & -050959.93 \\
J0221.5-0402 & J0221.5-0402a & 022130.20 & -040228.39 \\
& J0221.5-0402b & 022127.44 & -040233.69 \\
J0224.3-0505 & J0224.3-0505 & 022419.99 & -050525.03 \\
& J0224.3-0506 & 022418.19 & -050604.19 \\
J0224.4-0425 & J0224.4-0425 & 022421.45 & -042548.22 \\
& J0224.3-0425 & 022420.47 & -042542.94 \\
J0226.1-0459 & J0226.1-0459a & 022604.56 & -045932.41 \\
& J0226.1-0459b & 022603.65 & -045902.42 \\
J0227.5-0411 & J0227.6-0411 & 022735.55 & -041122.83 \\
& J0227.5-0411 & 022730.45 & -041116.20 \\
J0228.1-0408 & J0228.2-0408 & 022811.29 & -040842.76 \\
& J0228.1-0408 & 022808.72 & -040849.35 \\
J0228.4-0435 & J0228.4-0434 & 022825.38 & -043439.56 \\
& J0228.4-0435 & 022822.18 & -043504.12 \\
J0229.0-0504 & J0229.0-0505 & 022858.57 & -050521.97 \\
& J0229.0-0504 & 022857.44 & -050448.92 \\
J0229.1-0507 & J0229.1-0507a & 022908.64 & -050724.59 \\
& J0229.1-0507b & 022905.75 & -050739.30 \\
\hline & & &
\end{tabular}


C. Tasse et al.: The optical and infrared properties of low-frequency radio sources in the XMM-LSS field, Online Material p 2

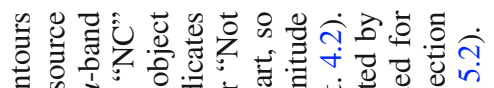

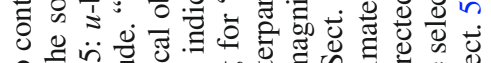

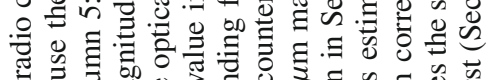

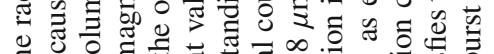

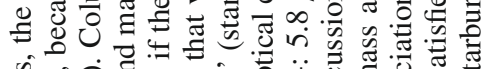

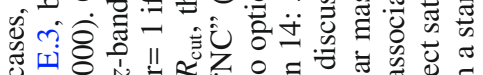

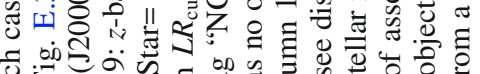

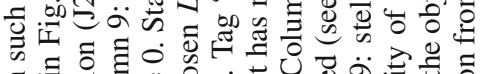

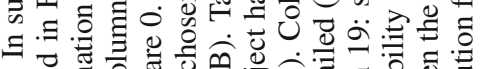

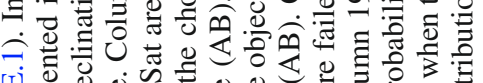

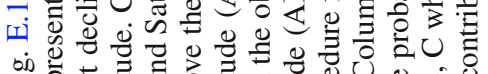

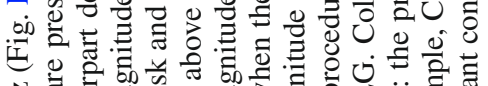

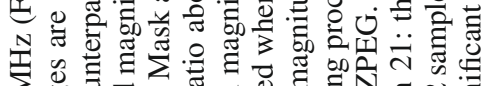

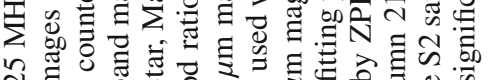

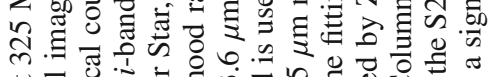

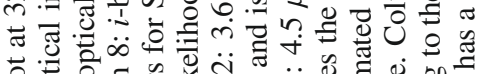

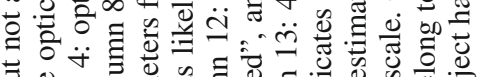

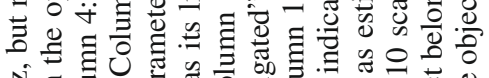

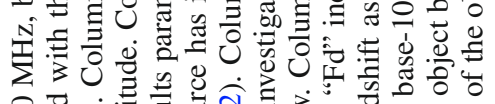
응.

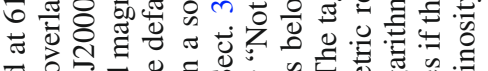

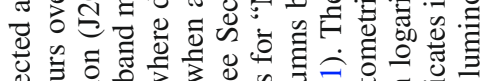

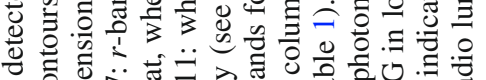

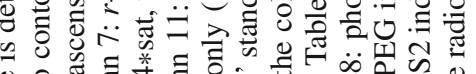
ญ。

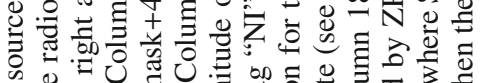

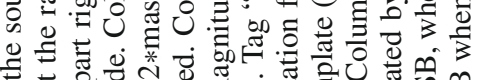

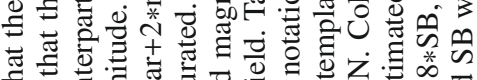

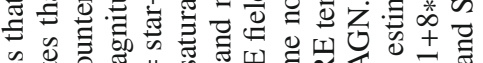

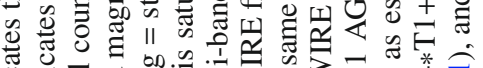

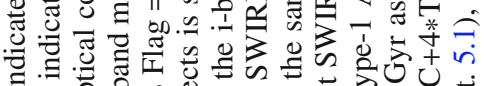

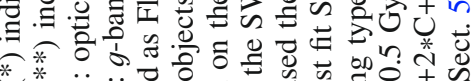

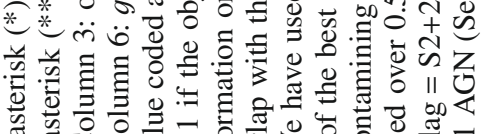

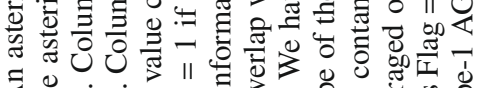

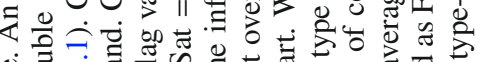

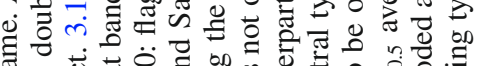

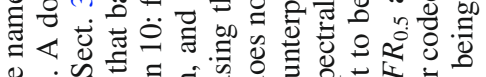
ठㄴ.

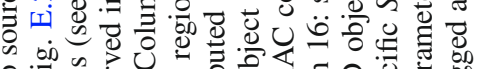

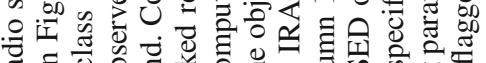

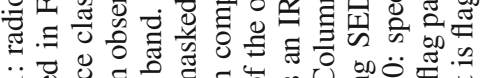

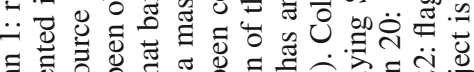

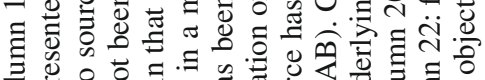

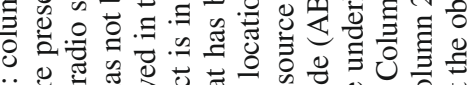

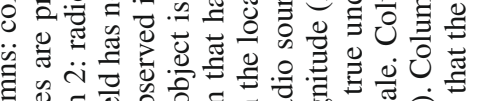

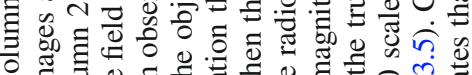

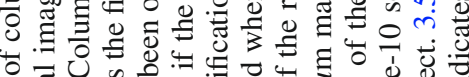

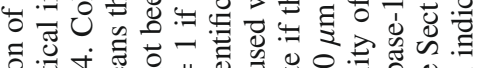

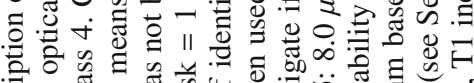

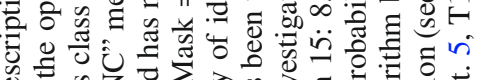

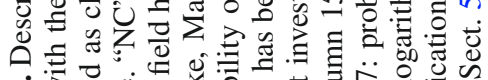

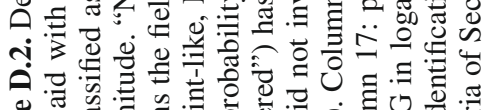

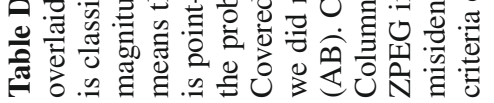

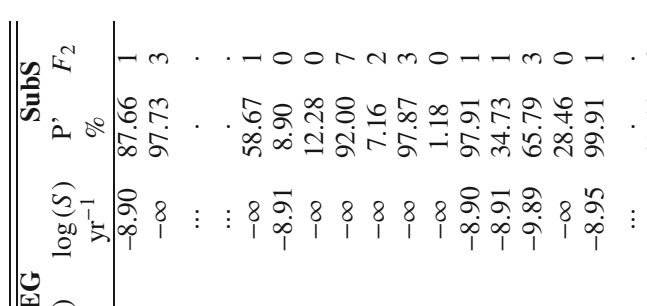

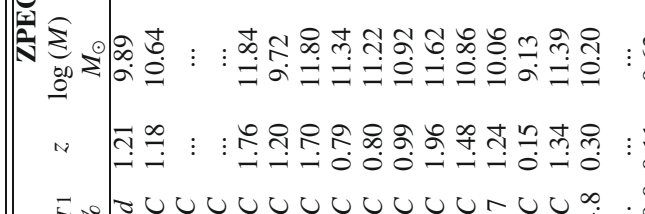

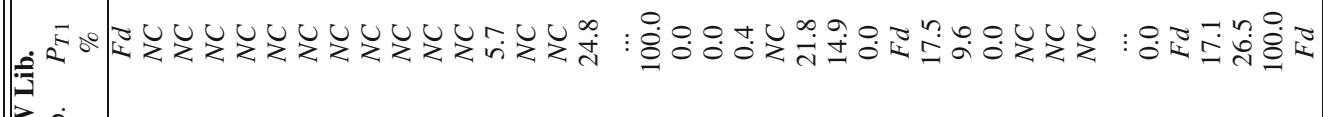

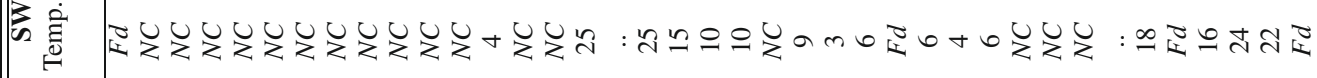

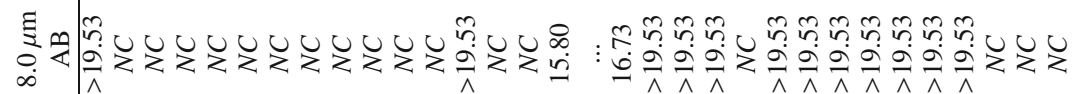

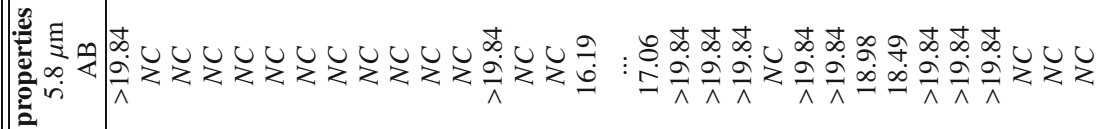

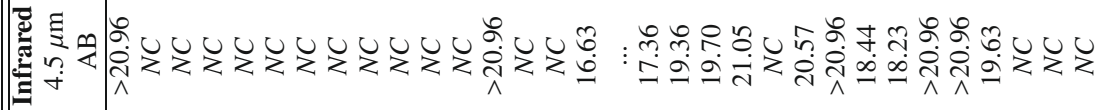

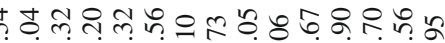

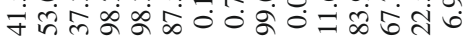
กิ่

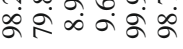

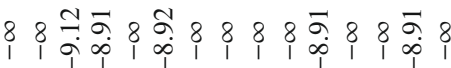

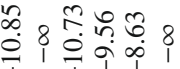

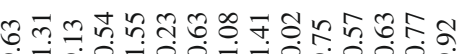

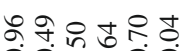

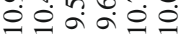
: $0000-0$

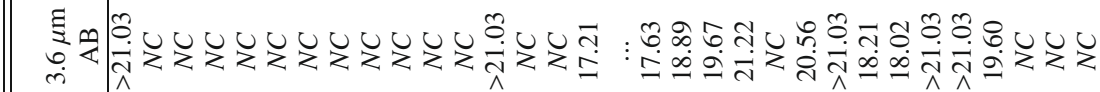

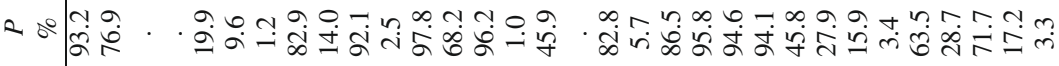

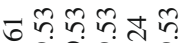

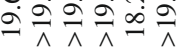
吕

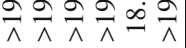

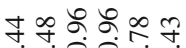
유슛ㅅㅇ

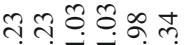
윷ㅅㅅㅅㅇㅅㅠ ป் $=00.00000000000 t .000000 n N n N 00000.0000-n$ ,

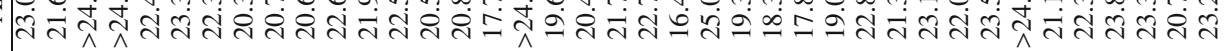
๓

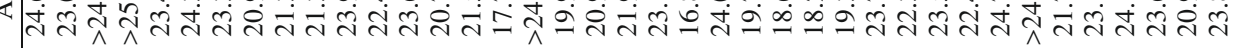
q

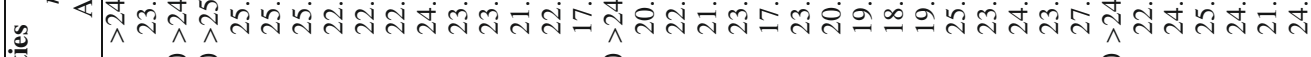
。

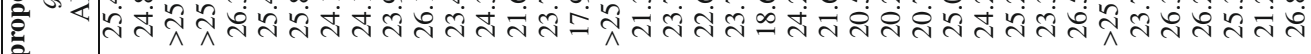
死 =

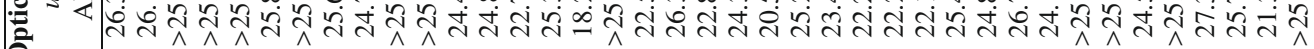

- के के

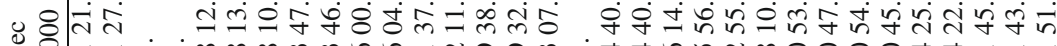

氙的

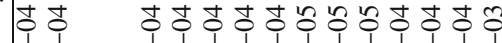

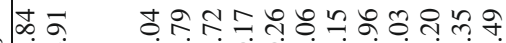

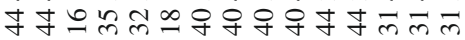

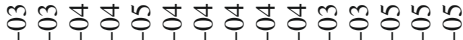
은

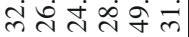

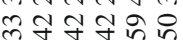

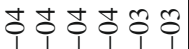
约

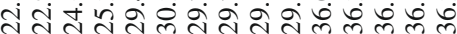
$\varangle 8$

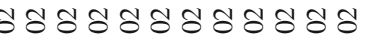
nnnnnn

ธำㄴำ응

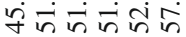
느는

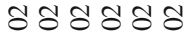

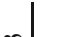

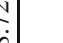$$
1
$$

莎

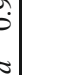


C. Tasse et al.: The optical and infrared properties of low-frequency radio sources in the XMM-LSS field, Online Material p 3

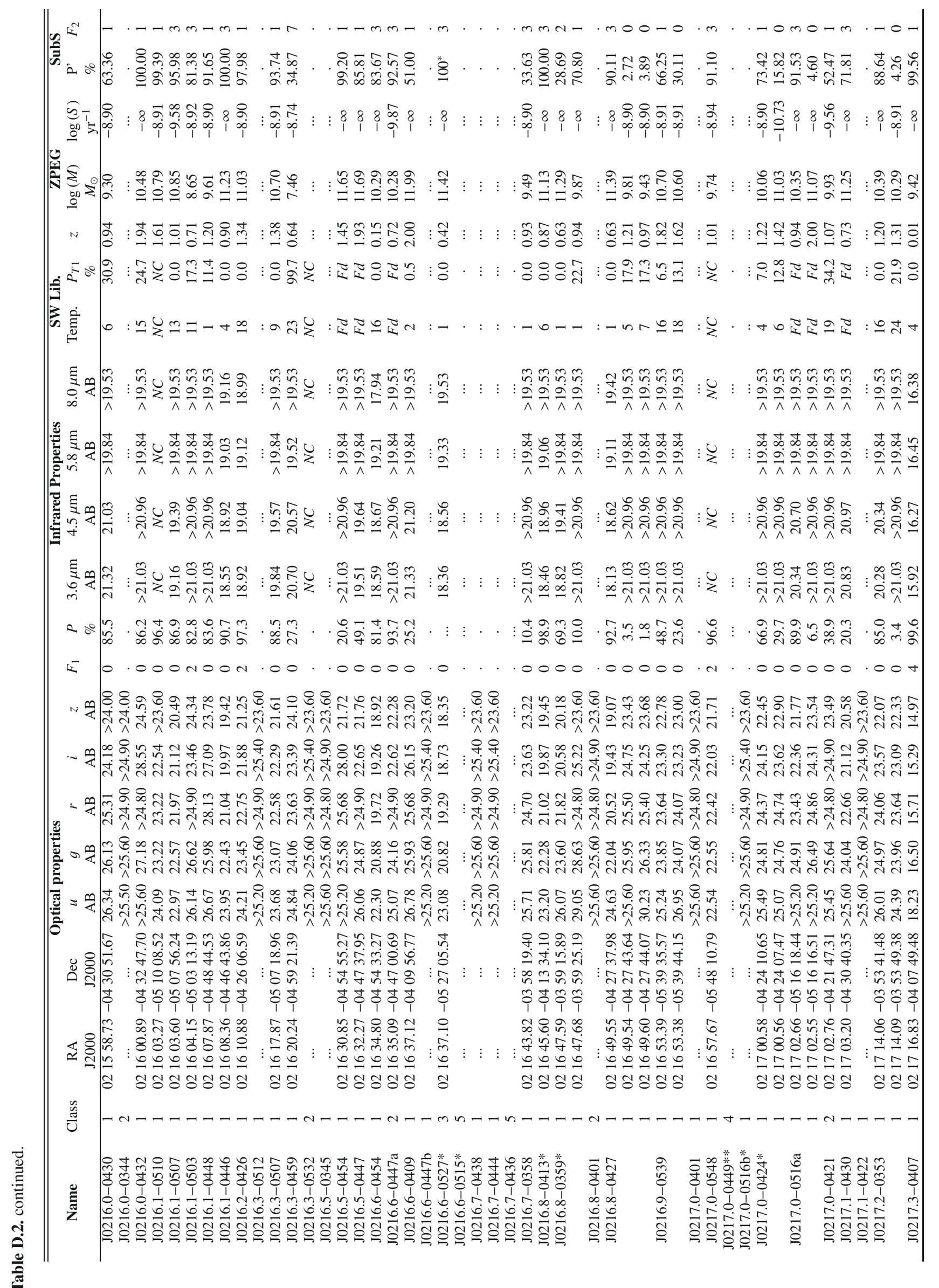


C. Tasse et al.: The optical and infrared properties of low-frequency radio sources in the XMM-LSS field, Online Material p 4

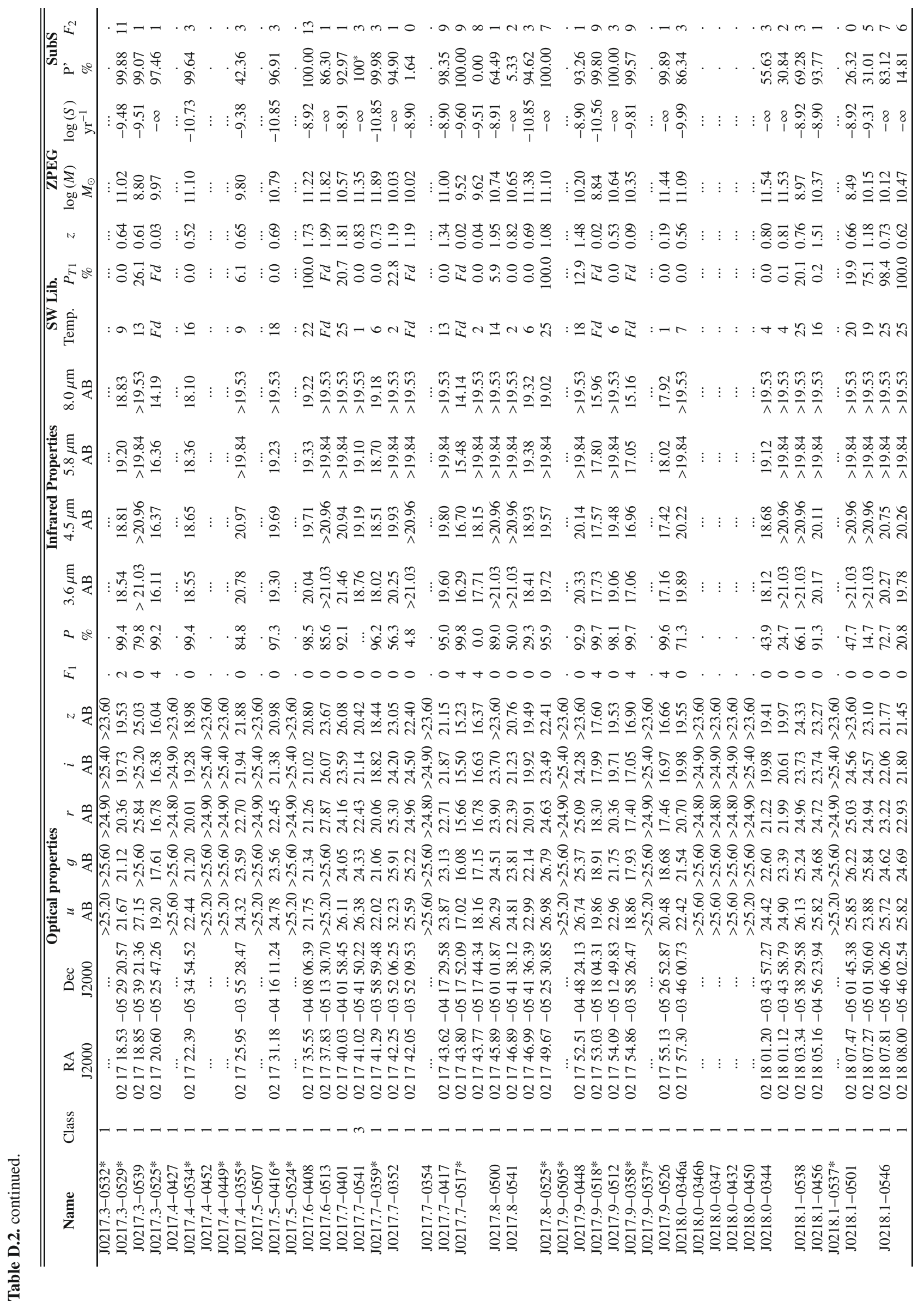


C. Tasse et al.: The optical and infrared properties of low-frequency radio sources in the XMM-LSS field, Online Material p 5

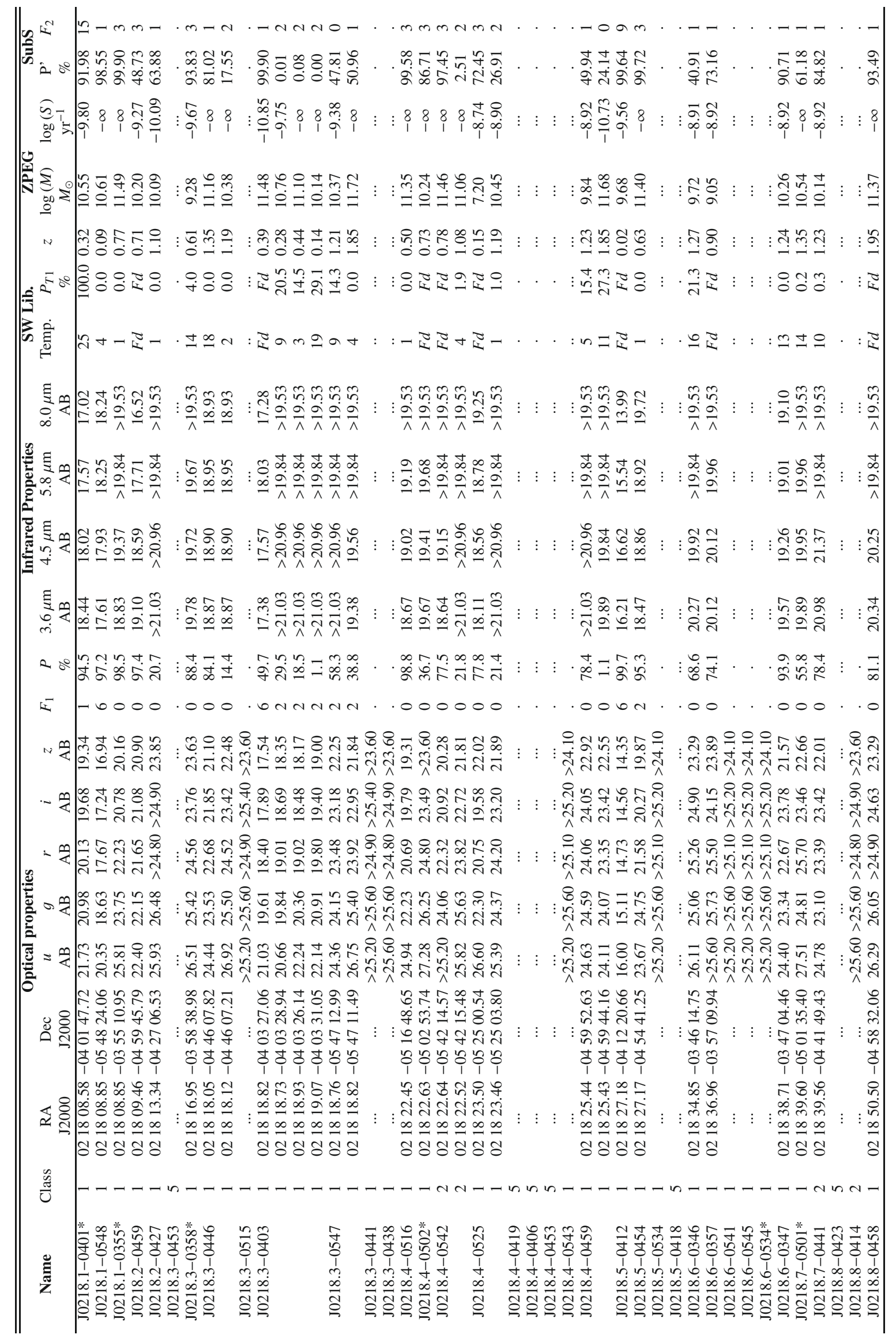




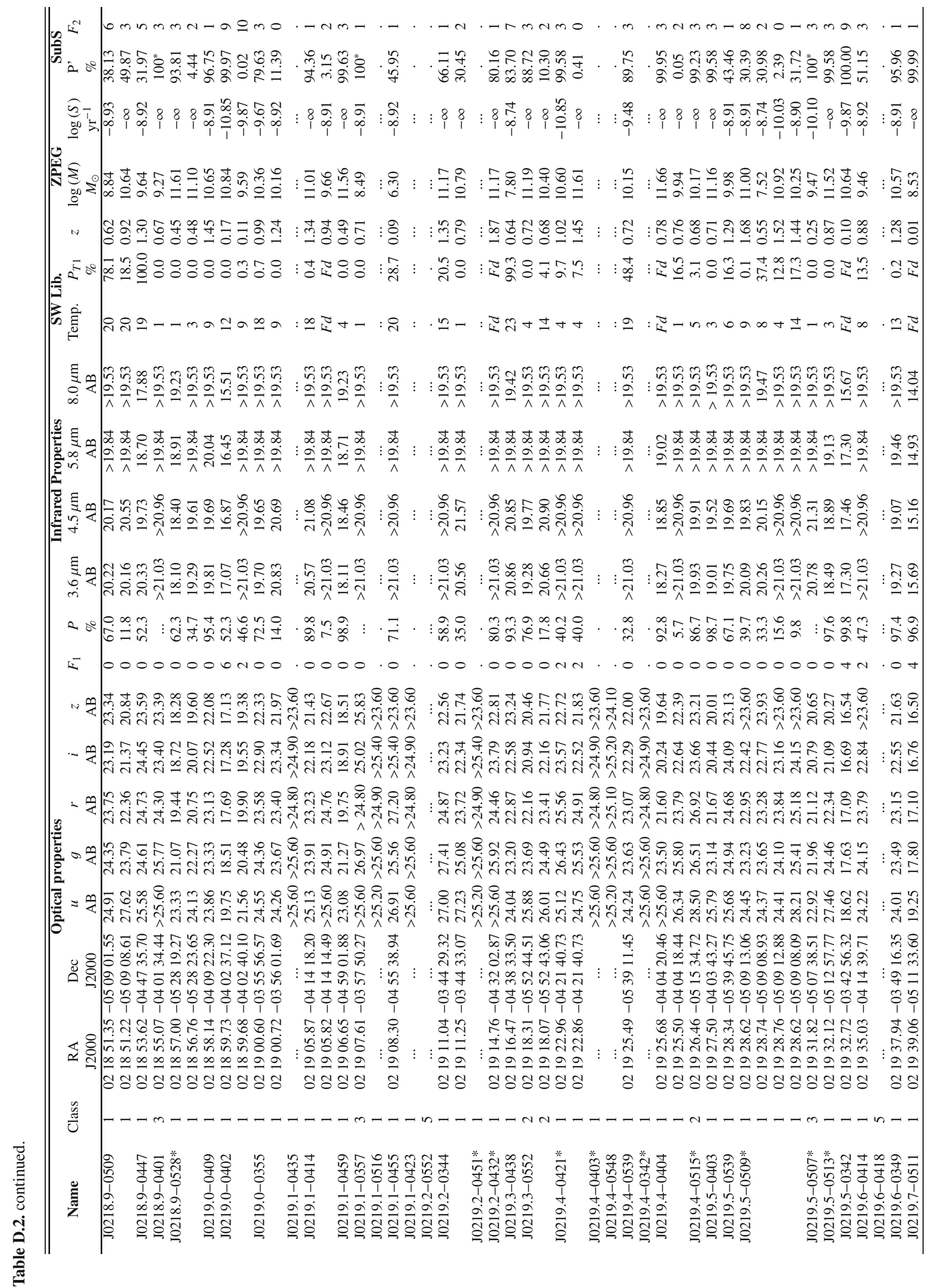


C. Tasse et al.: The optical and infrared properties of low-frequency radio sources in the XMM-LSS field, Online Material p 7

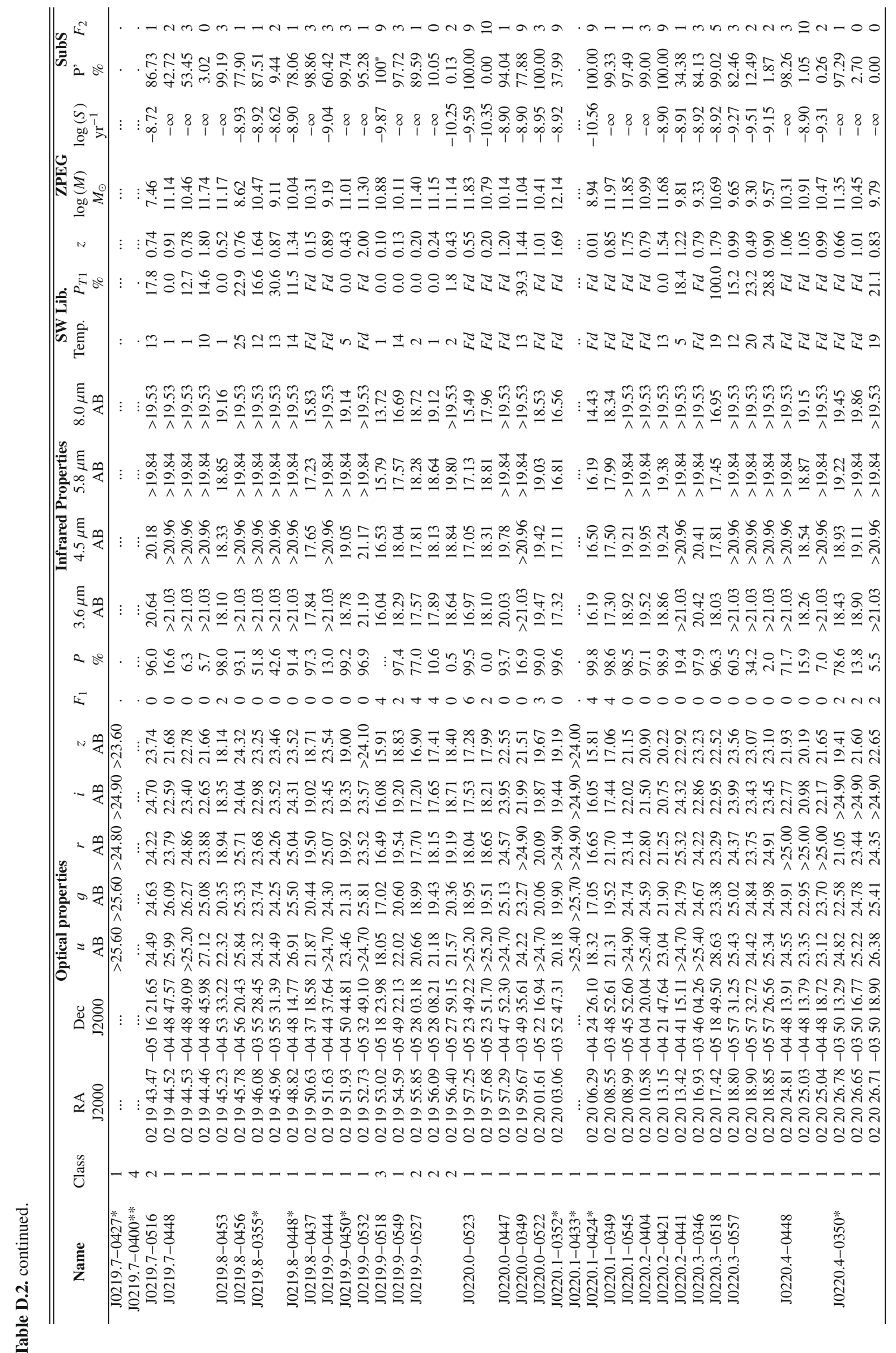


C. Tasse et al.: The optical and infrared properties of low-frequency radio sources in the XMM-LSS field, Online Material $p 8$

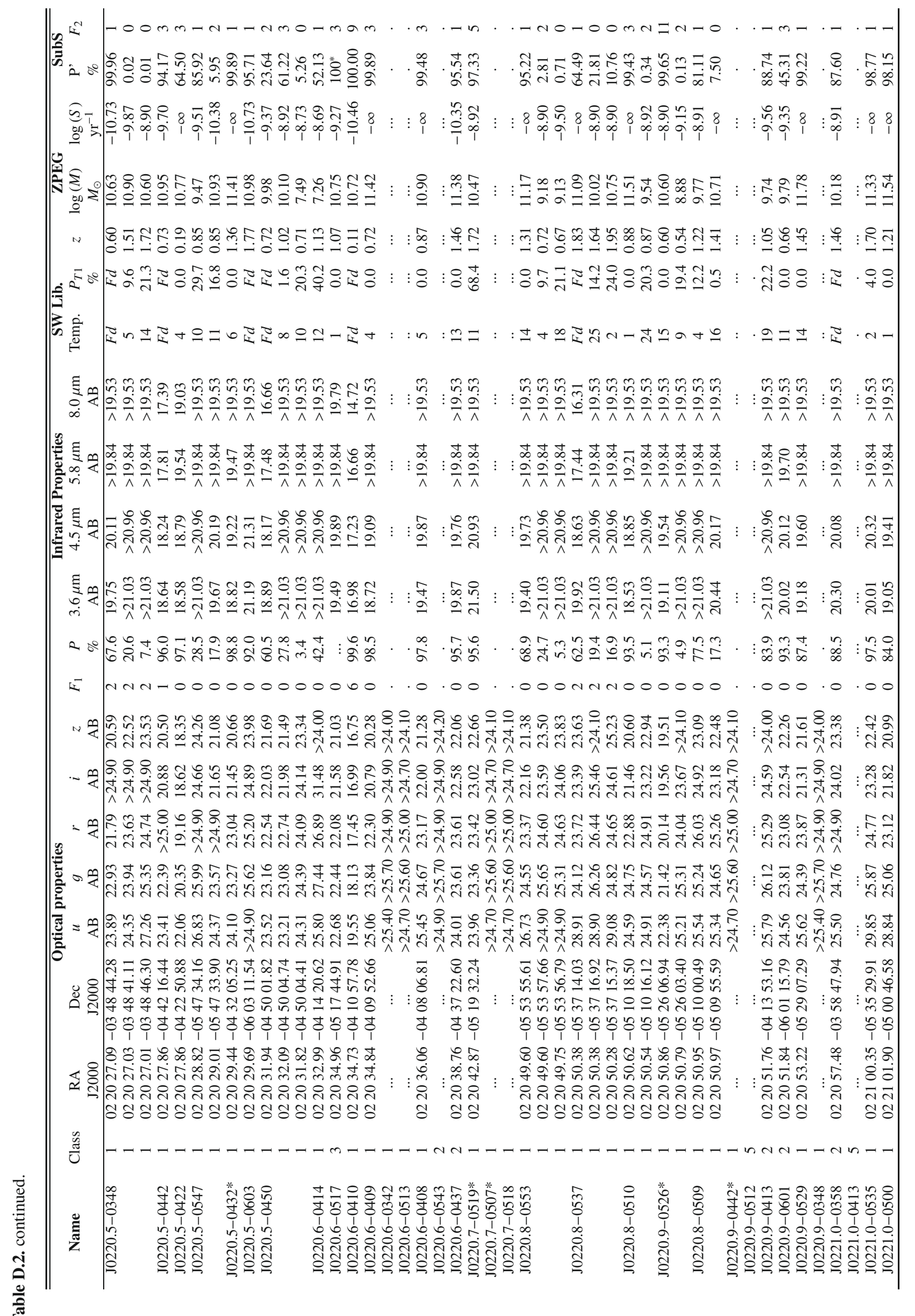


C. Tasse et al.: The optical and infrared properties of low-frequency radio sources in the XMM-LSS field, Online Material p 9

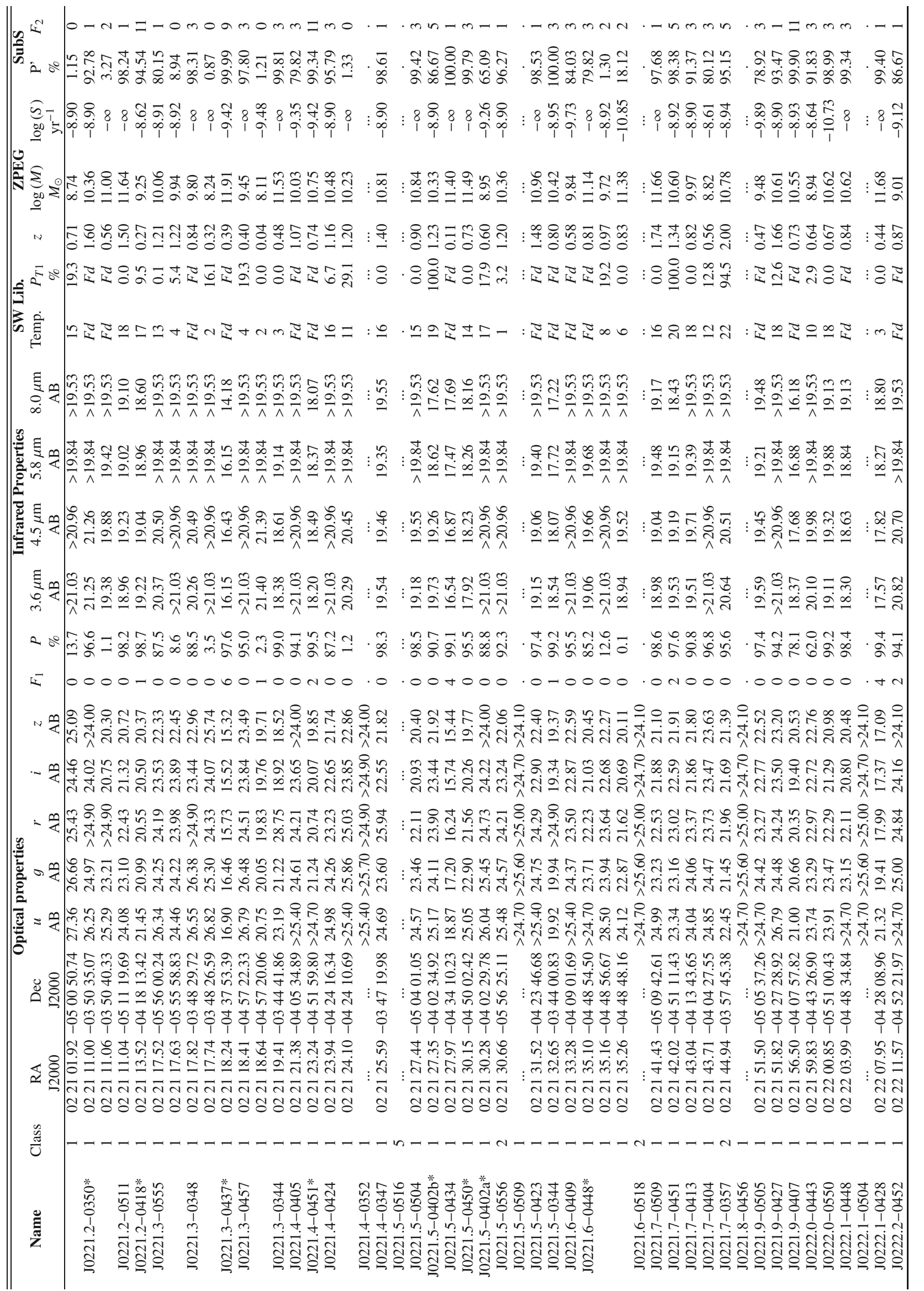


C. Tasse et al.: The optical and infrared properties of low-frequency radio sources in the XMM-LSS field, Online Material p 10

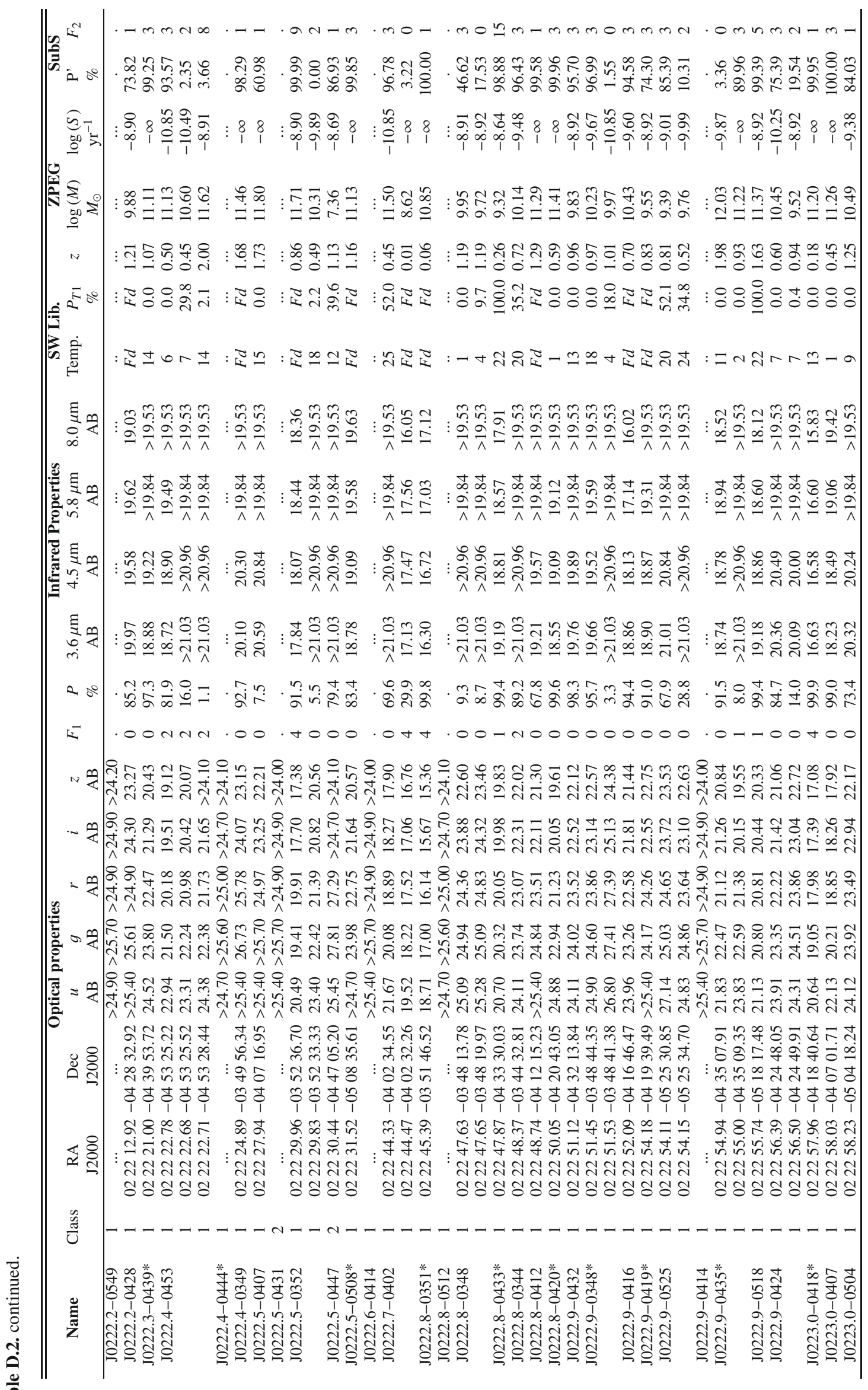




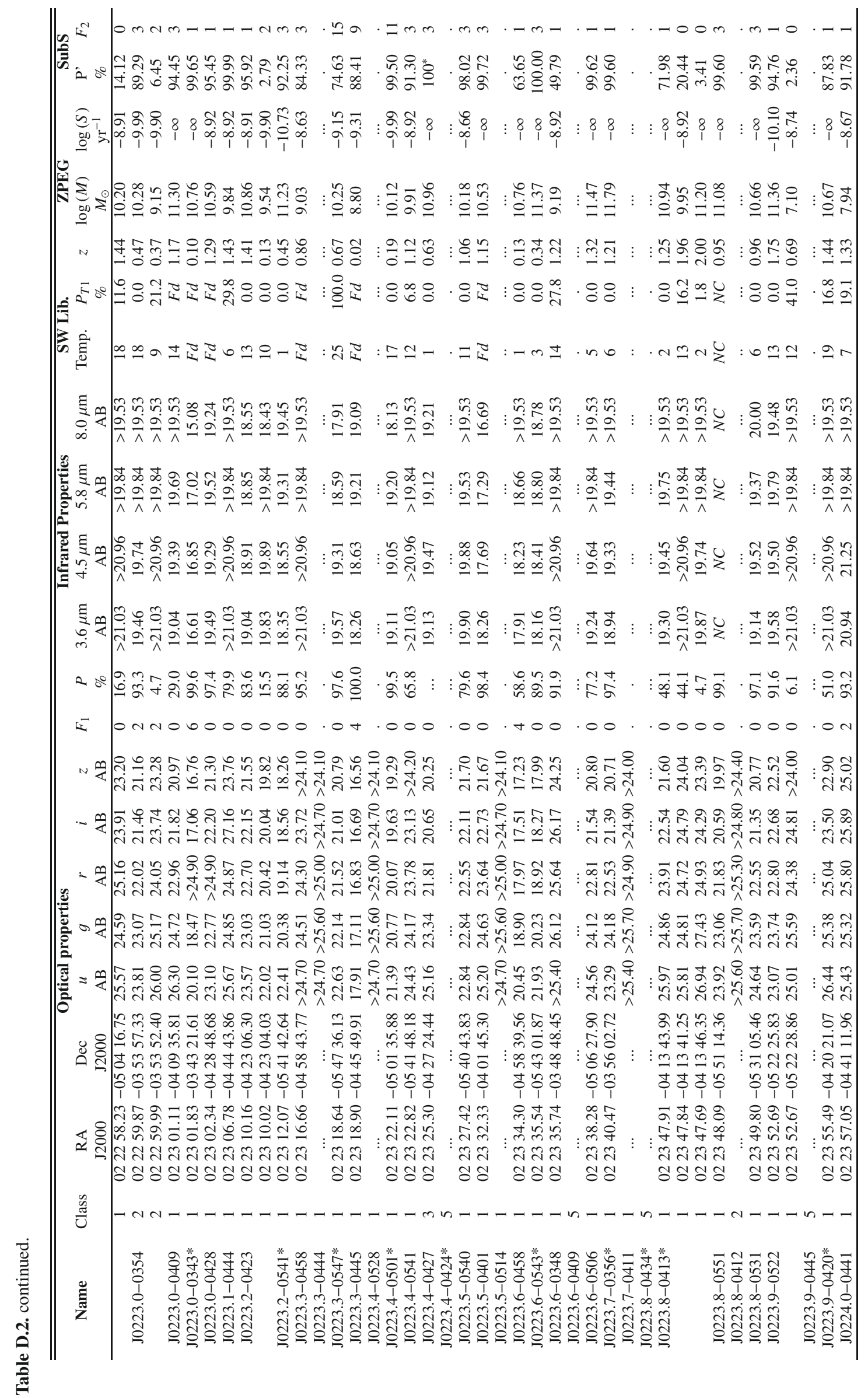


C. Tasse et al.: The optical and infrared properties of low-frequency radio sources in the XMM-LSS field, Online Material p 12

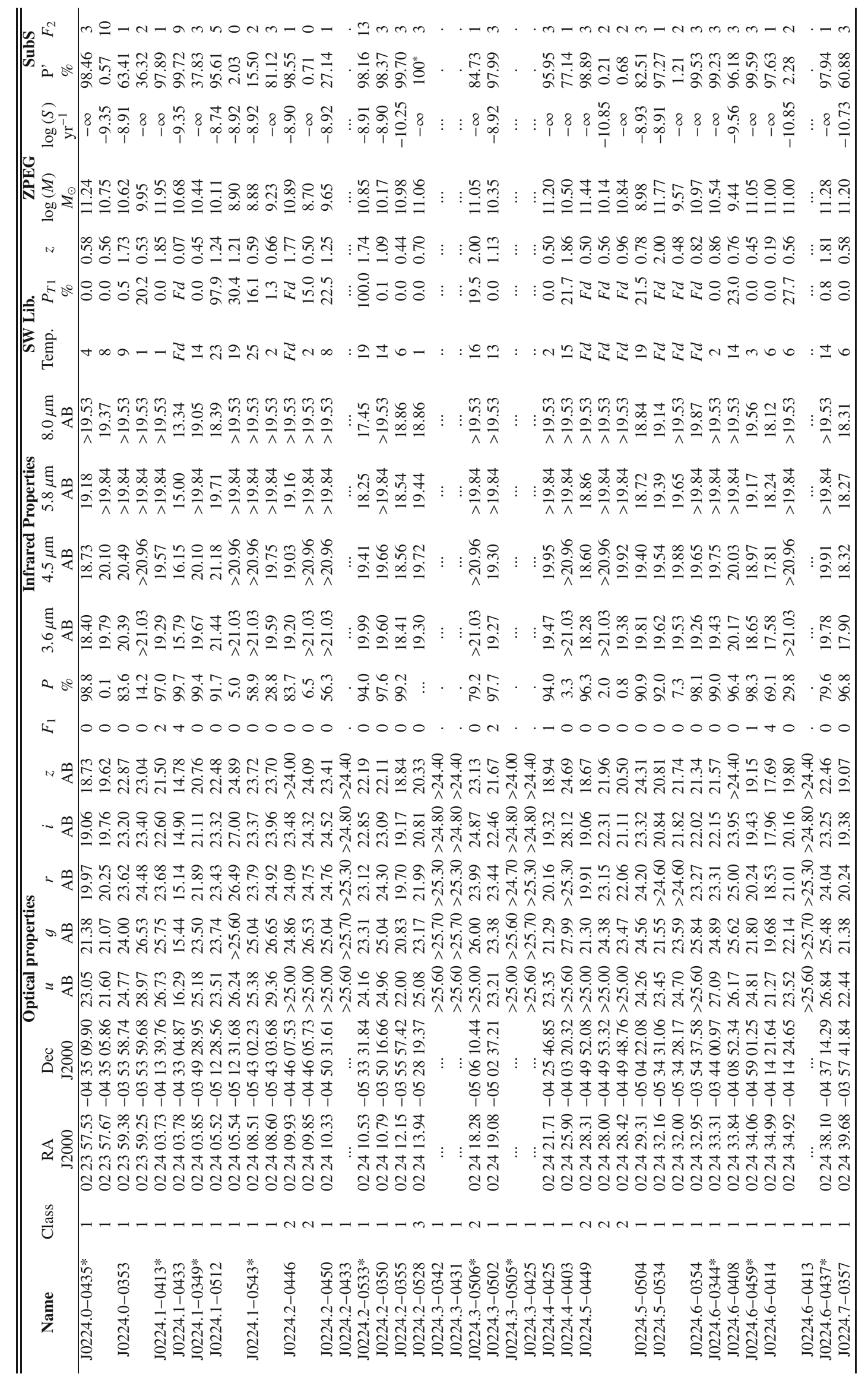




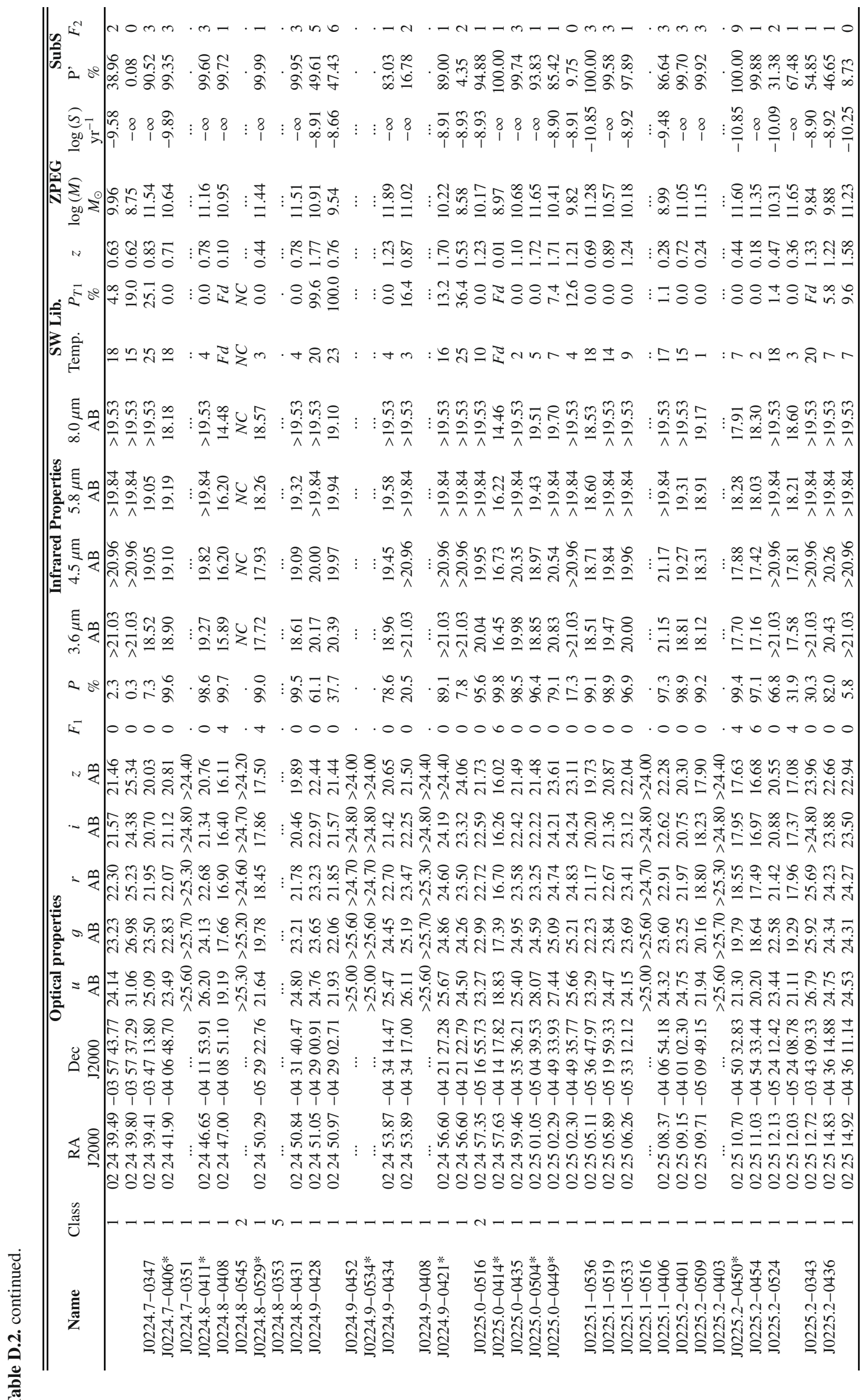




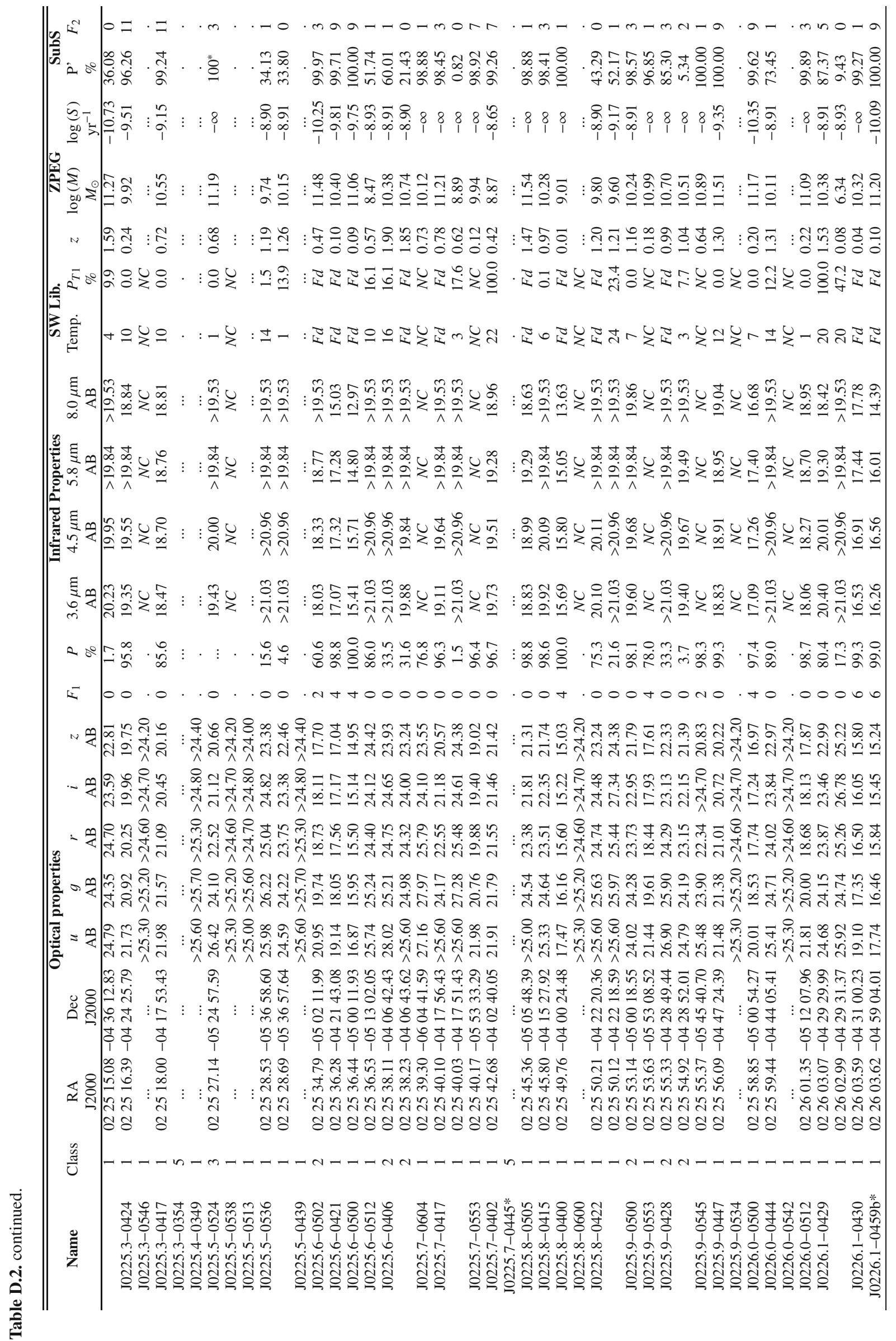




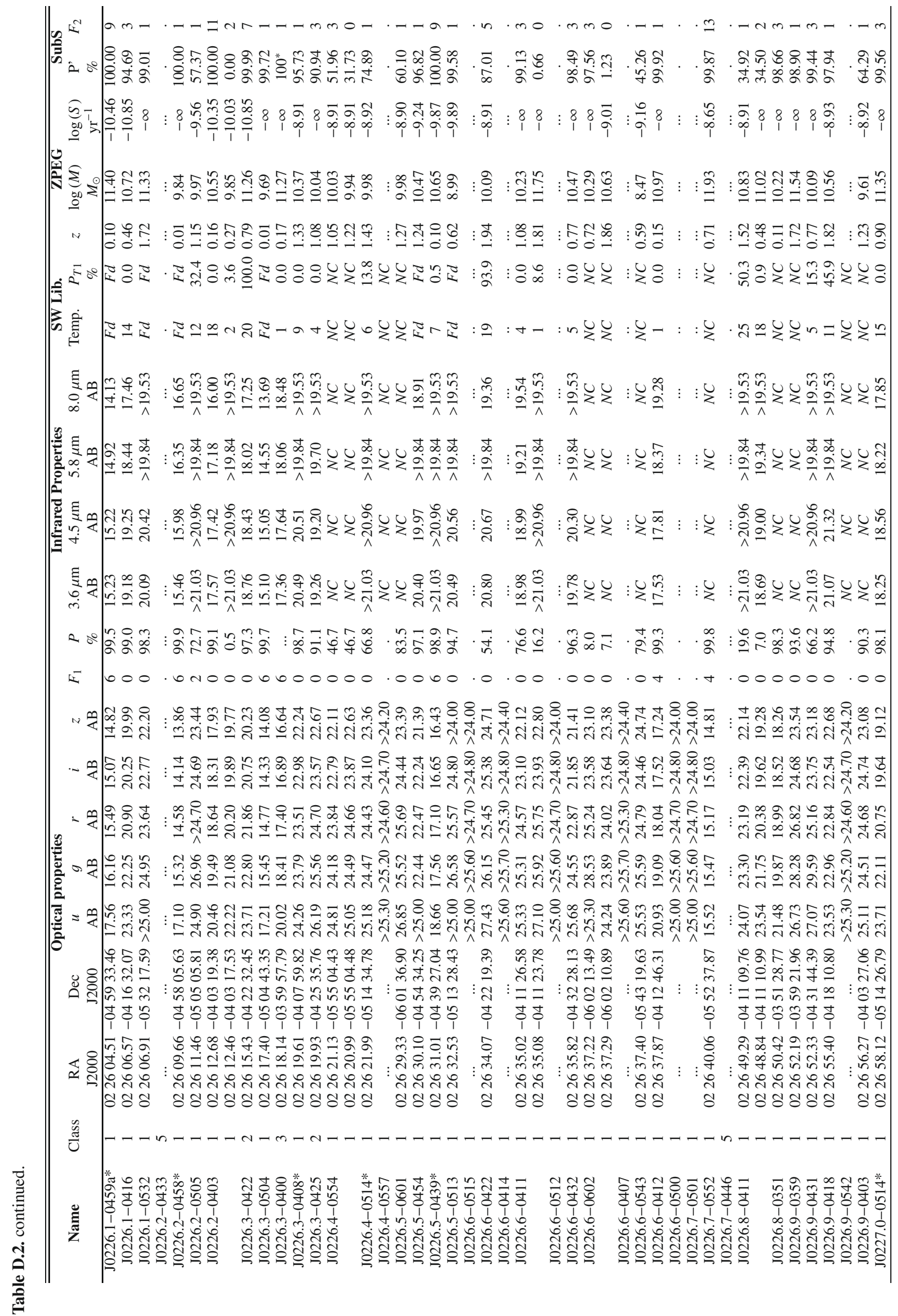


C. Tasse et al.: The optical and infrared properties of low-frequency radio sources in the XMM-LSS field, Online Material $p 16$

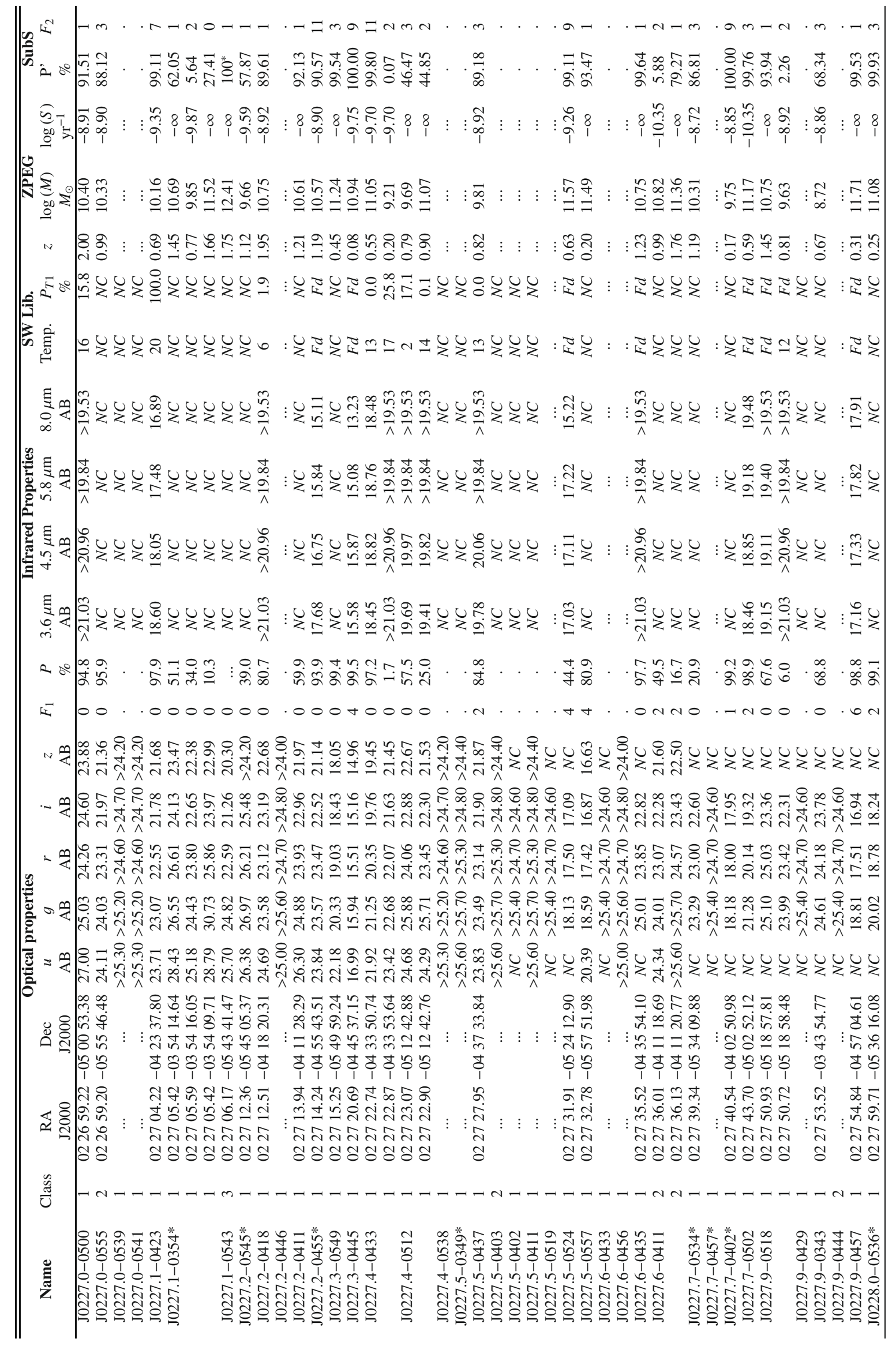




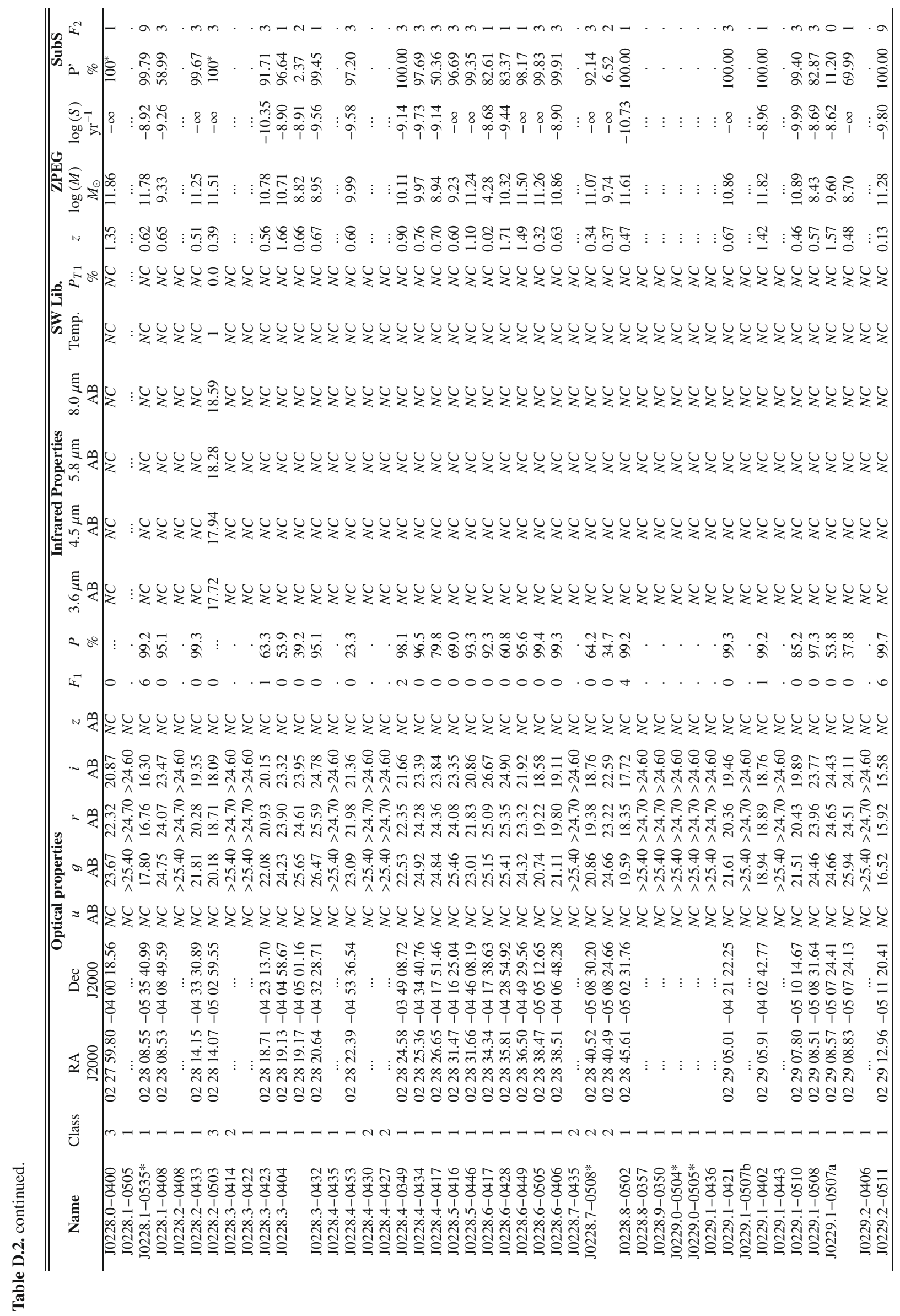


C. Tasse et al.: The optical and infrared properties of low-frequency radio sources in the XMM-LSS field, Online Material p 18

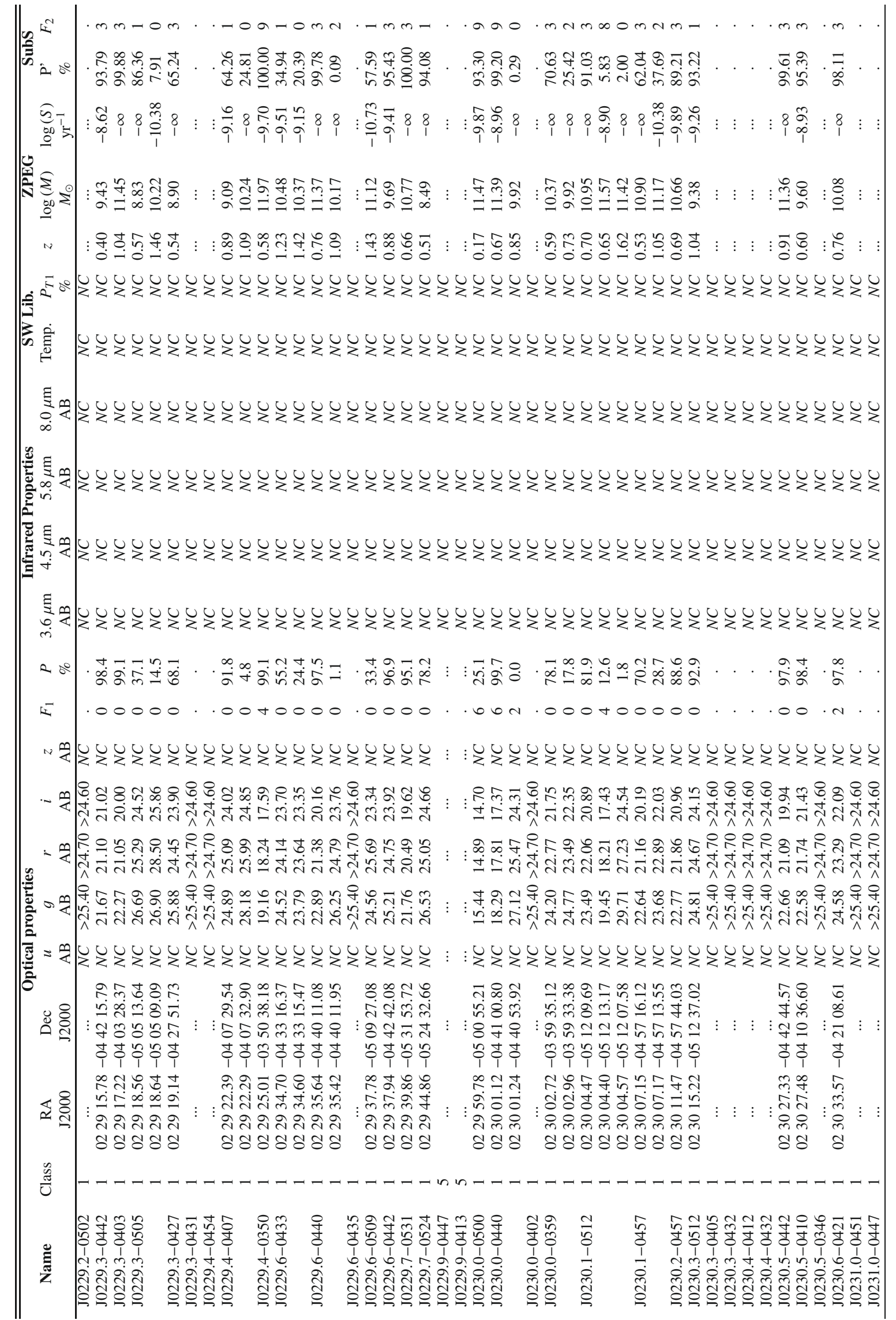


C. Tasse et al.: The optical and infrared properties of low-frequency radio sources in the XMM-LSS field, Online Material p 19

\section{Appendix E: overlays}
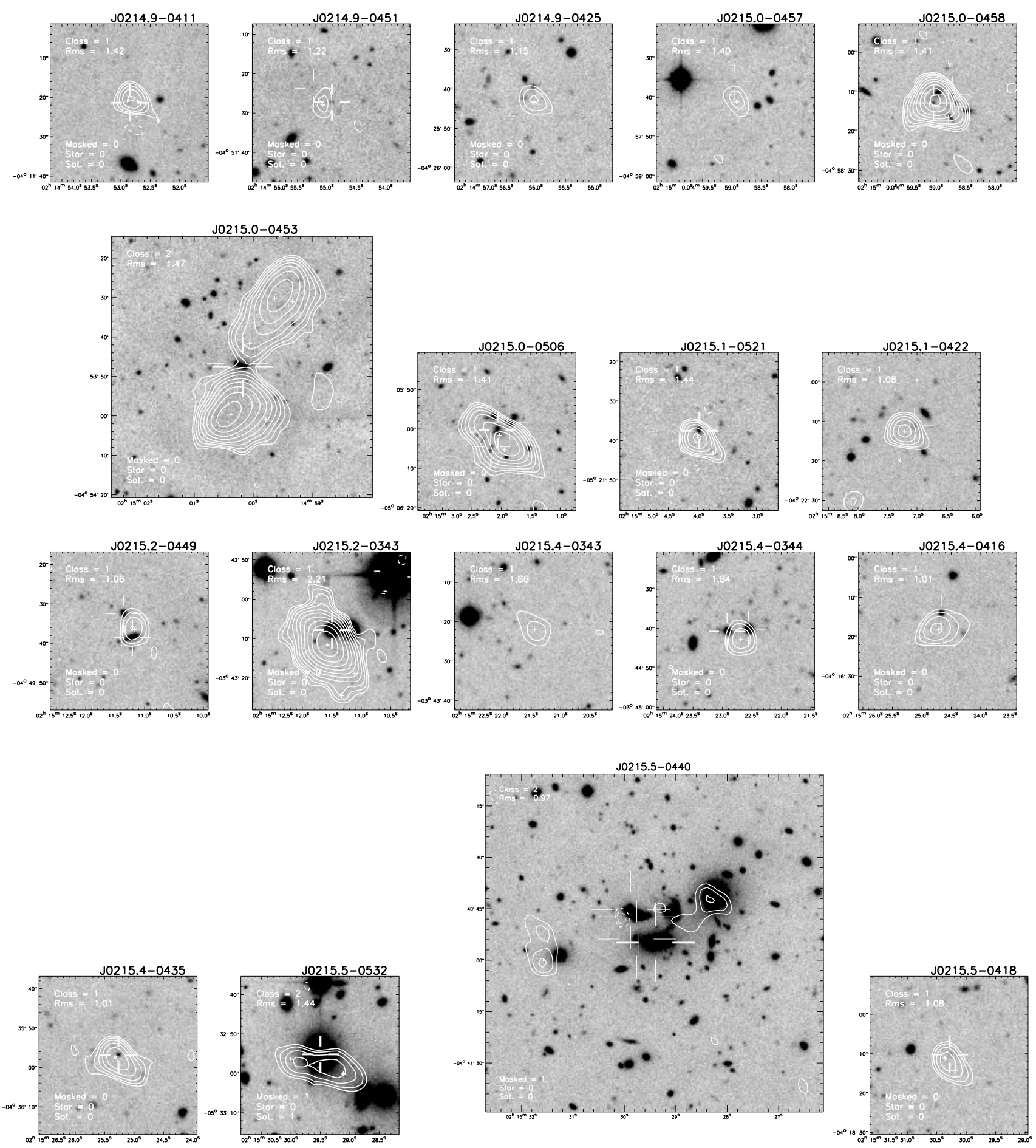

Fig. E.1. Greyscale: the $i$-band image. Contours: The $325 \mathrm{MHz}$ contours drawn at levels of $3 \sigma_{\text {local }} \times 1,1.4,2,2.8,4, \ldots$. The local noise $\sigma_{\text {local }}$ appears in each overlay, and is in units of mJy.beam ${ }^{-1}$. The largest cross indicates the position of the optical identification with the highest identification probability, $p_{\max }$. The thickness of the other crosses scales as $p / p_{\max }$ where $p$ is the probability of identification of the given source. Small crosses indicate the positions of the Gaussian fitting components appearing in the Tasse et al. (2006) source list, used for the centroïd position calculation. On the bottom left corner of each image, the radio source morphological classification is given, as well as the flag status of the optical host in the optical catalog: Masked $=1$ if the objects is masked, Star $=1$ if it is point-like, and Sat. $=1$ if it is saturated. 
C. Tasse et al.: The optical and infrared properties of low-frequency radio sources in the XMM-LSS field, Online Material p 20
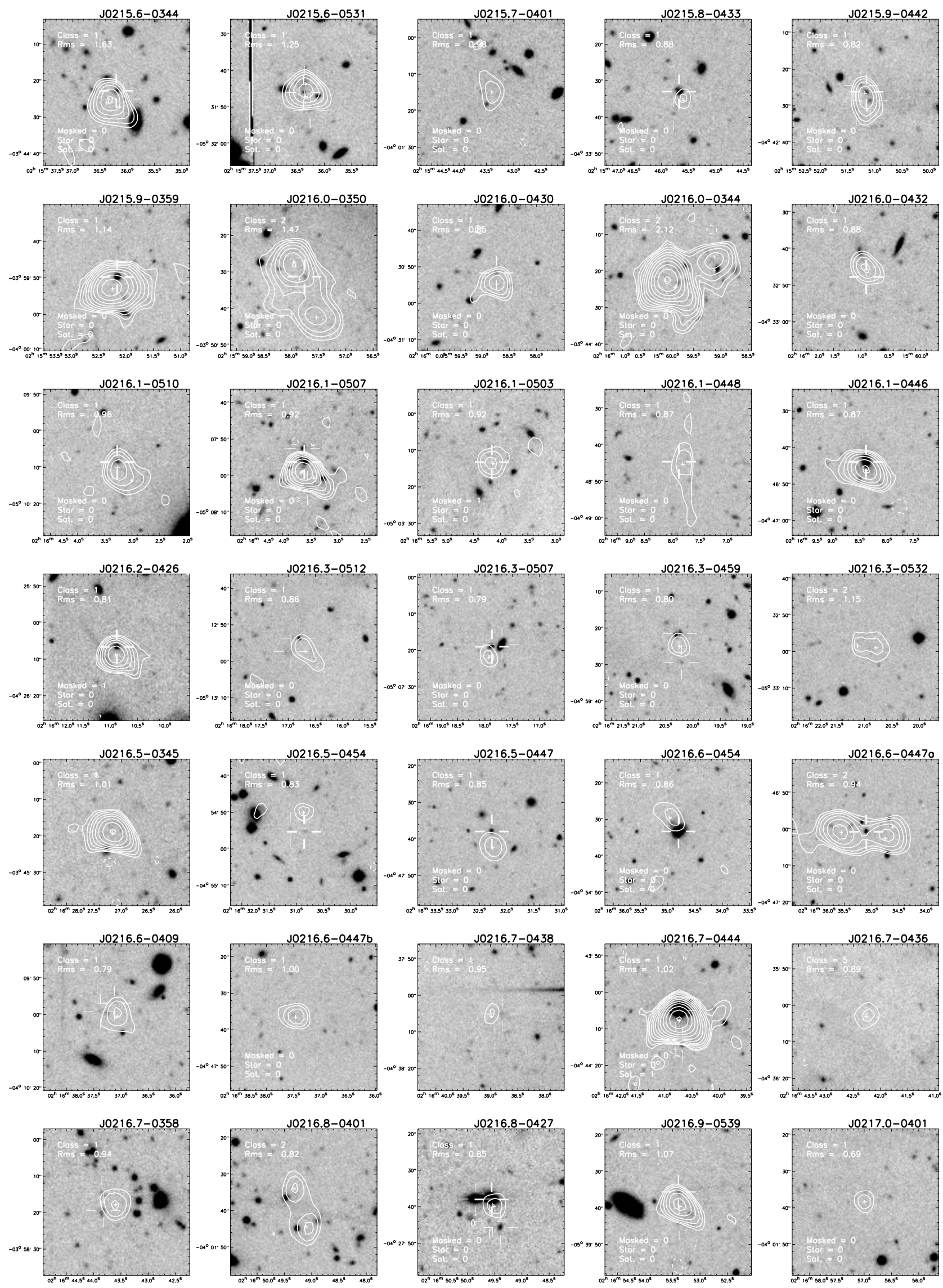

Fig. E.1. continued. 
C. Tasse et al.: The optical and infrared properties of low-frequency radio sources in the XMM-LSS field, Online Material p 21
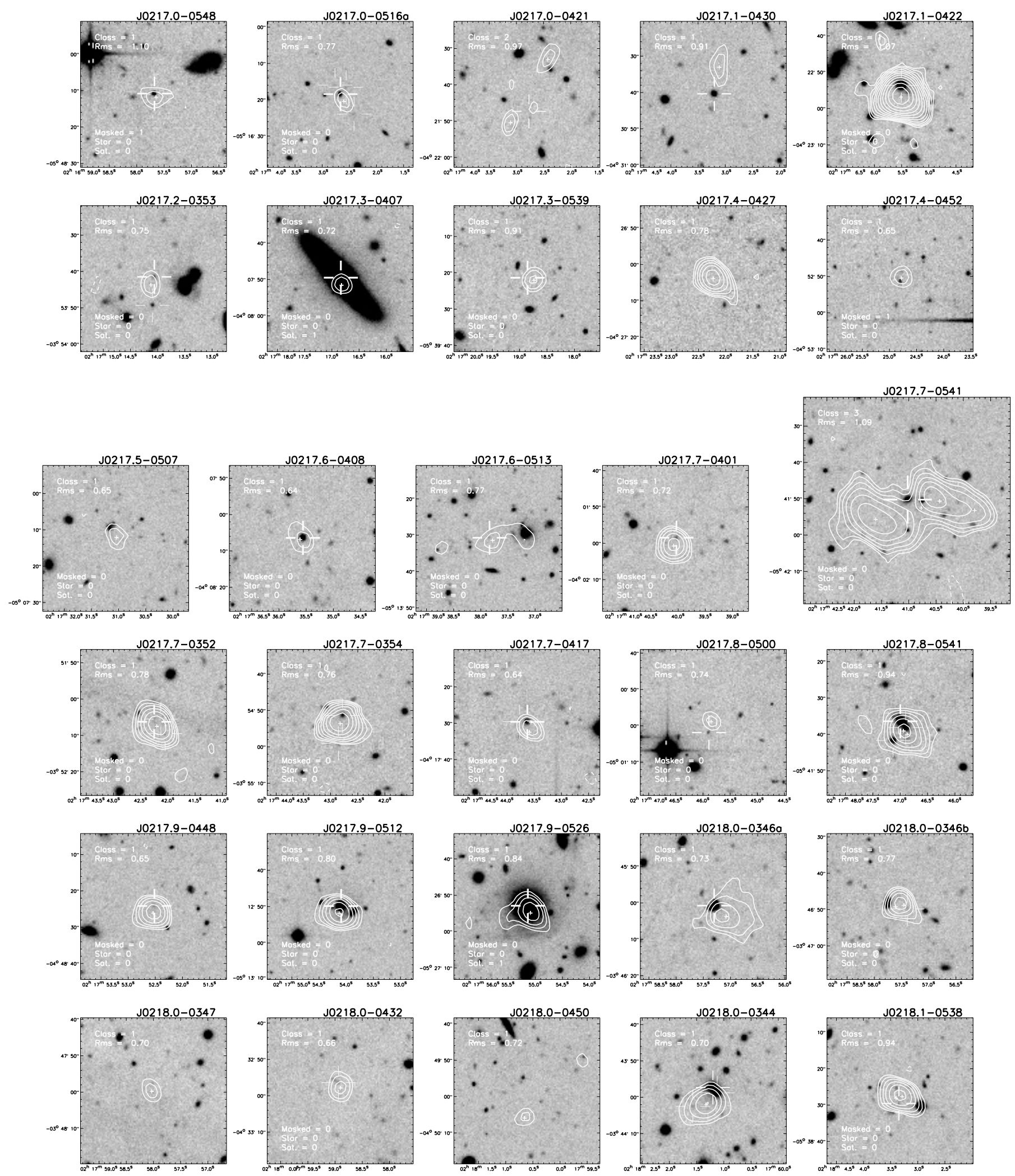

Fig. E.1. continued. 
C. Tasse et al.: The optical and infrared properties of low-frequency radio sources in the XMM-LSS field, Online Material p 22
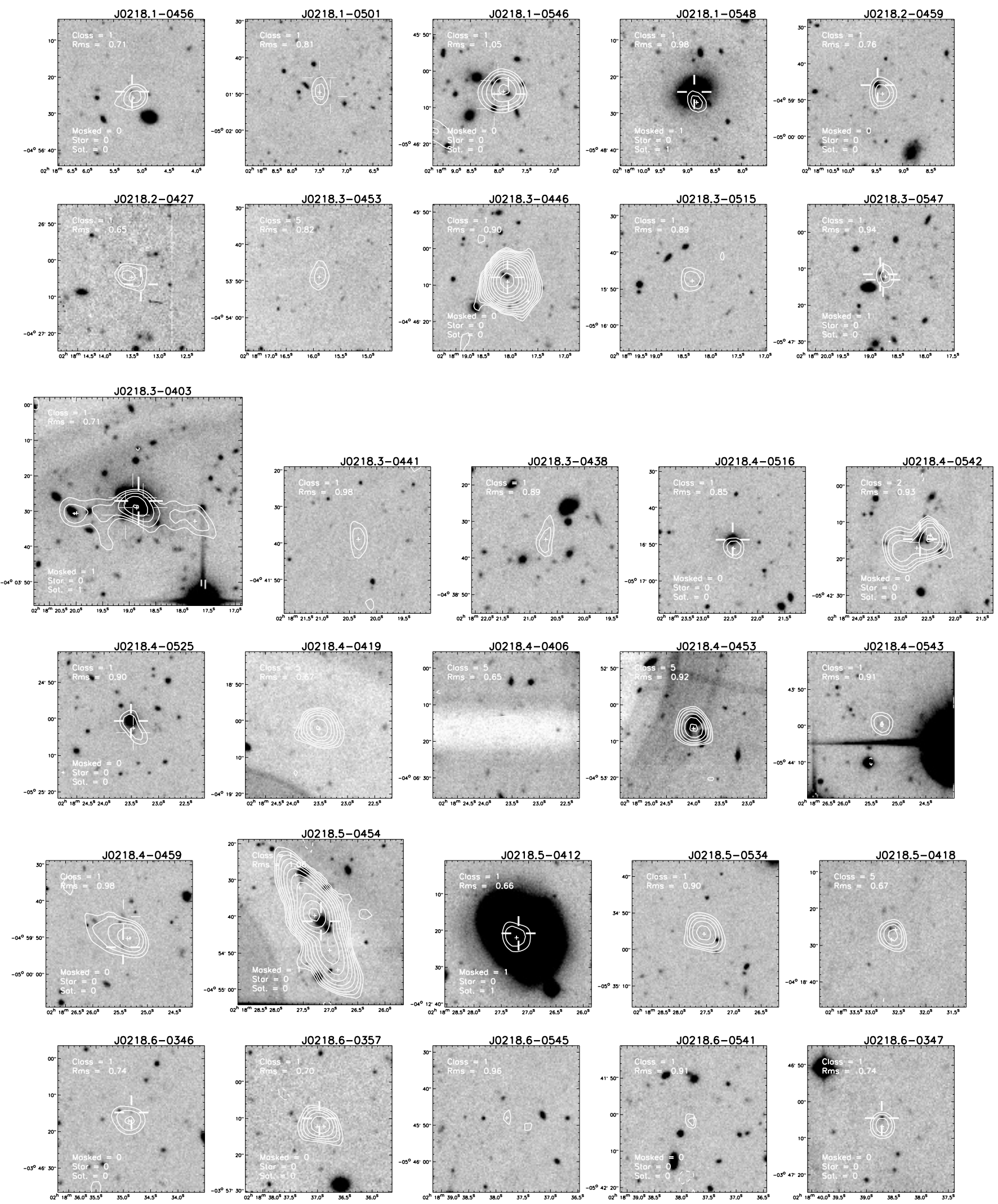

Fig. E.1. continued. 
C. Tasse et al.: The optical and infrared properties of low-frequency radio sources in the XMM-LSS field, Online Material p 23
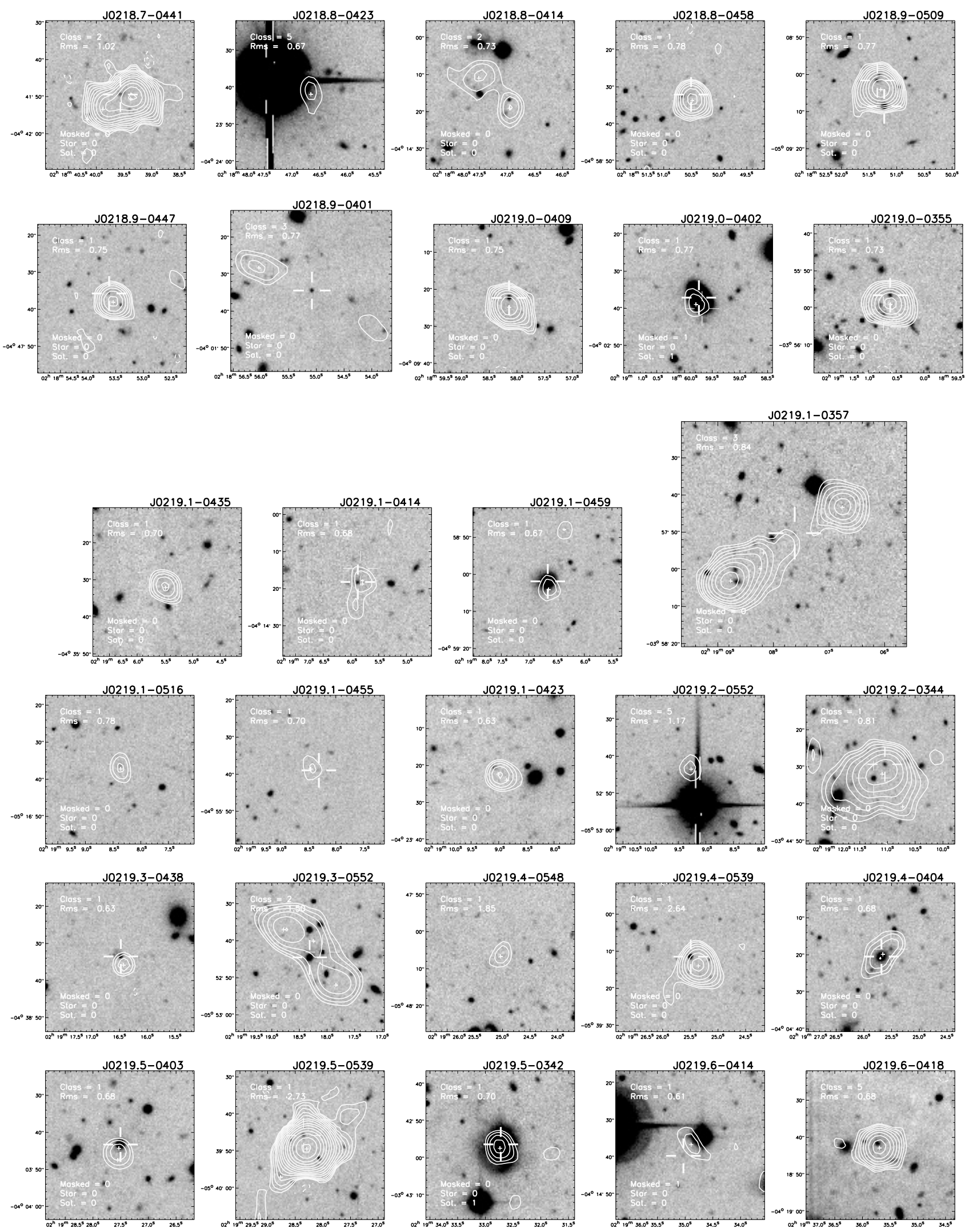

Fig. E.1. continued. 
C. Tasse et al.: The optical and infrared properties of low-frequency radio sources in the XMM-LSS field, Online Material p 24
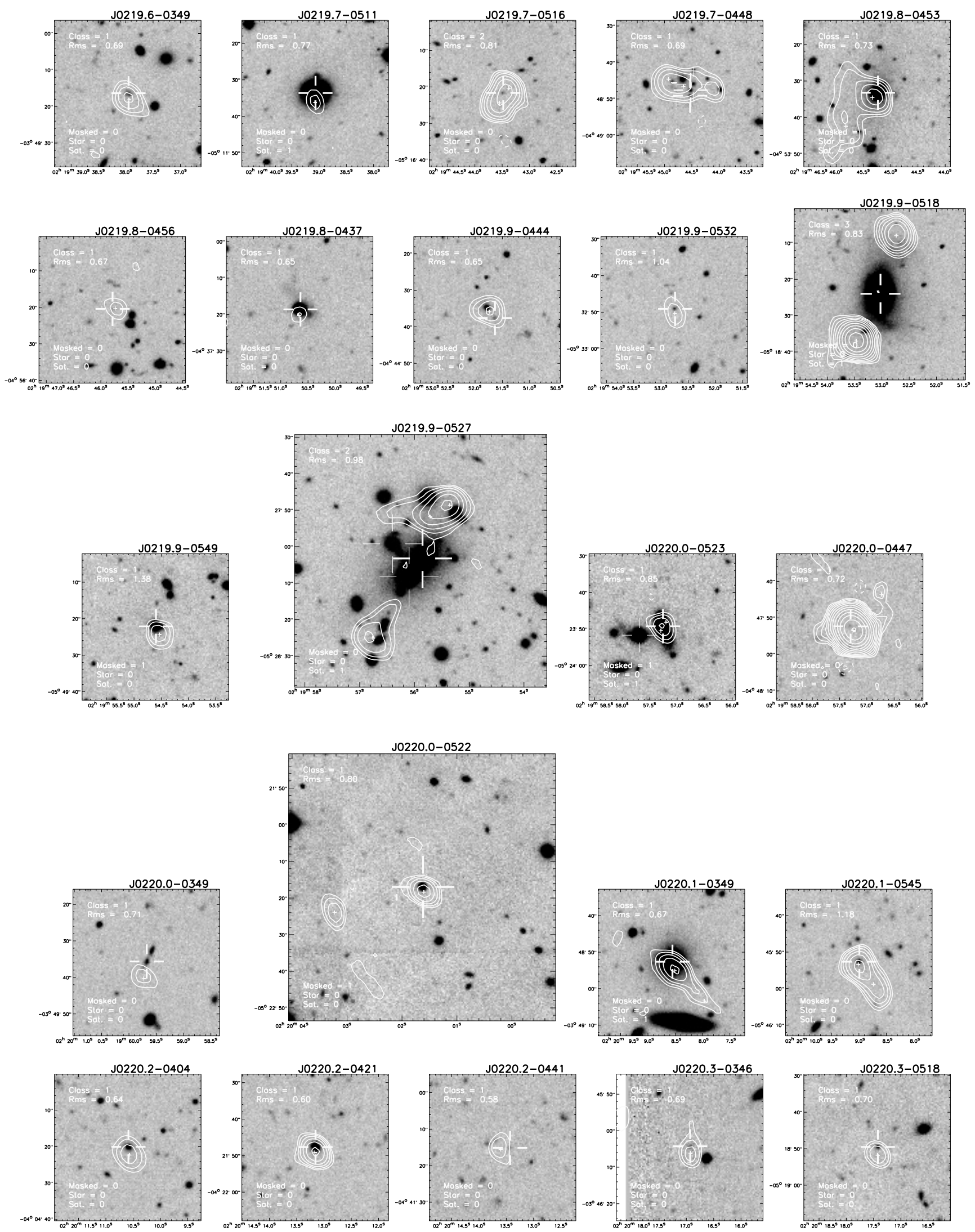

Fig. E.1. continued. 
C. Tasse et al.: The optical and infrared properties of low-frequency radio sources in the XMM-LSS field, Online Material p 25
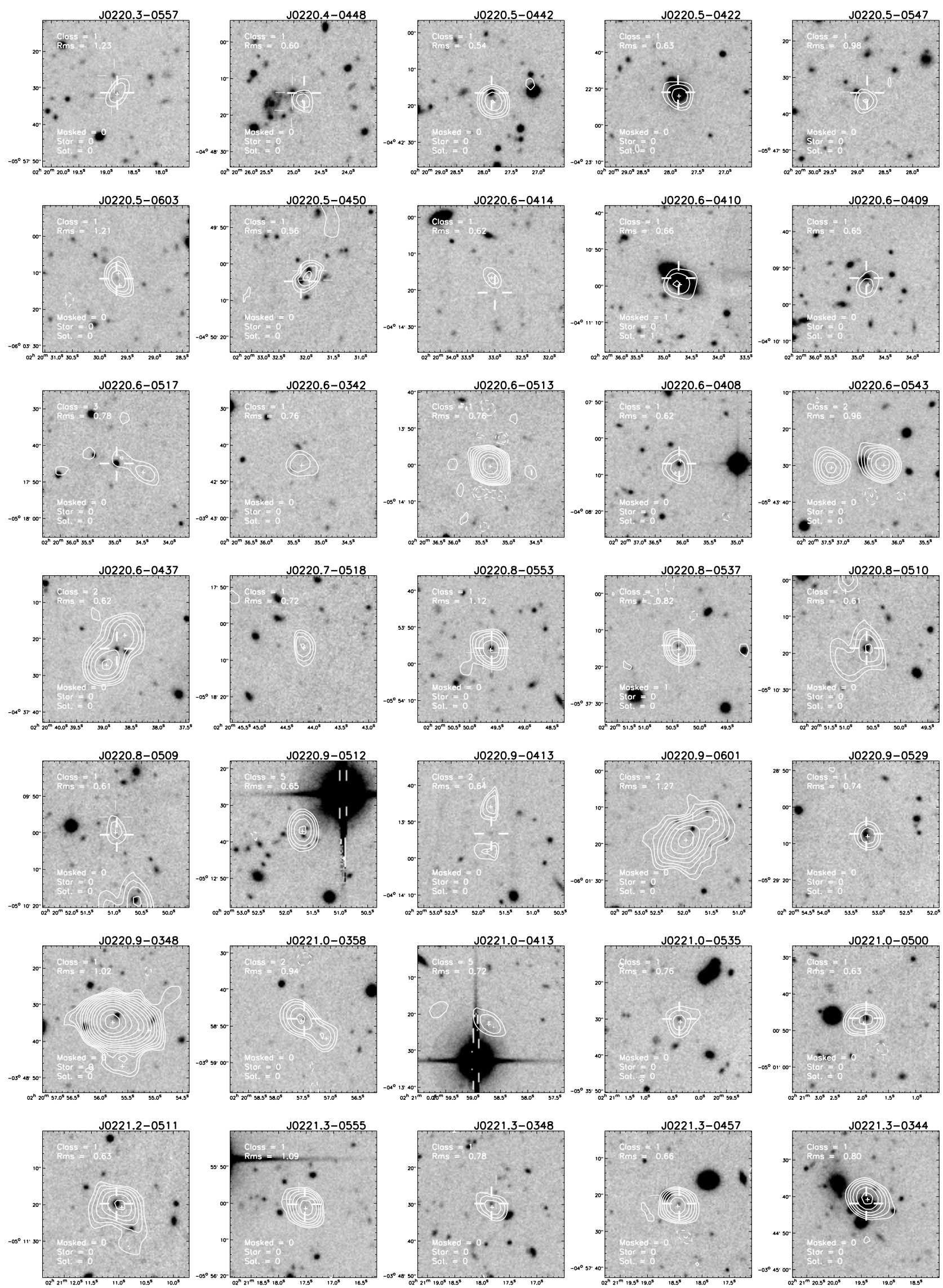

Fig. E.1. continued. 
C. Tasse et al.: The optical and infrared properties of low-frequency radio sources in the XMM-LSS field, Online Material p 26


Fig. E.1. continued. 
C. Tasse et al.: The optical and infrared properties of low-frequency radio sources in the XMM-LSS field, Online Material p 27
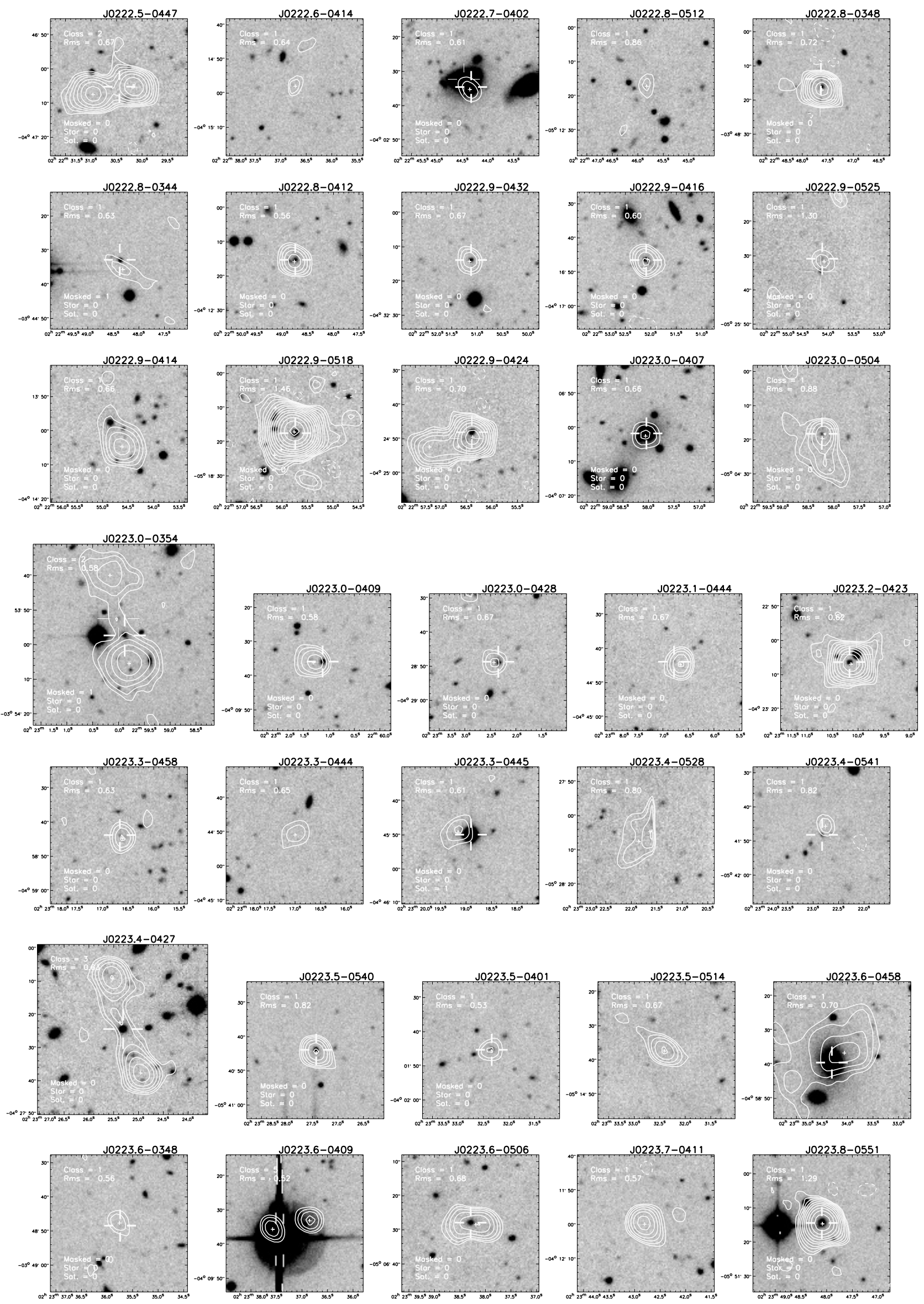

Fig. E.1. continued. 
C. Tasse et al.: The optical and infrared properties of low-frequency radio sources in the XMM-LSS field, Online Material p 28
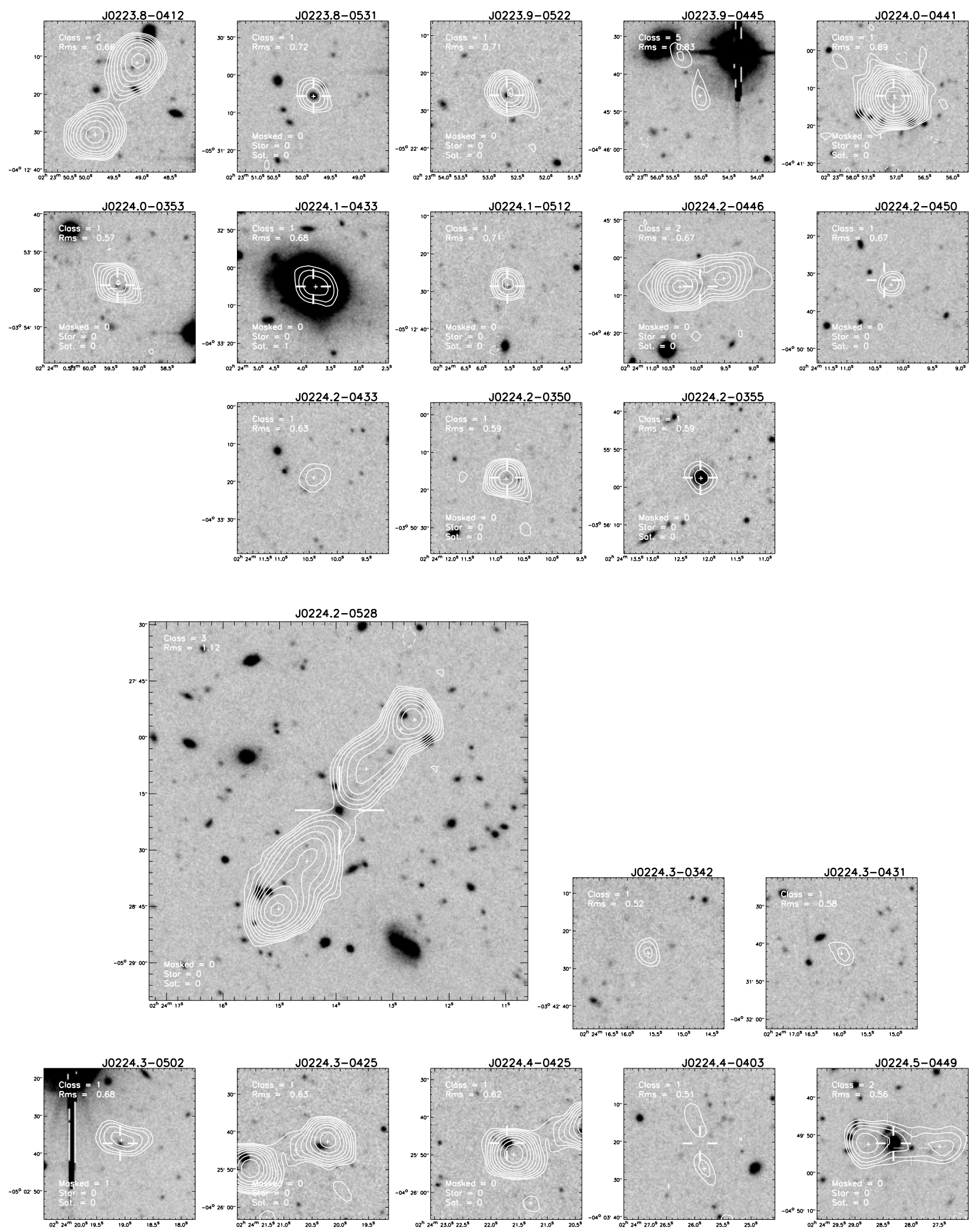

Fig. E.1. continued. 
C. Tasse et al.: The optical and infrared properties of low-frequency radio sources in the XMM-LSS field, Online Material p 29
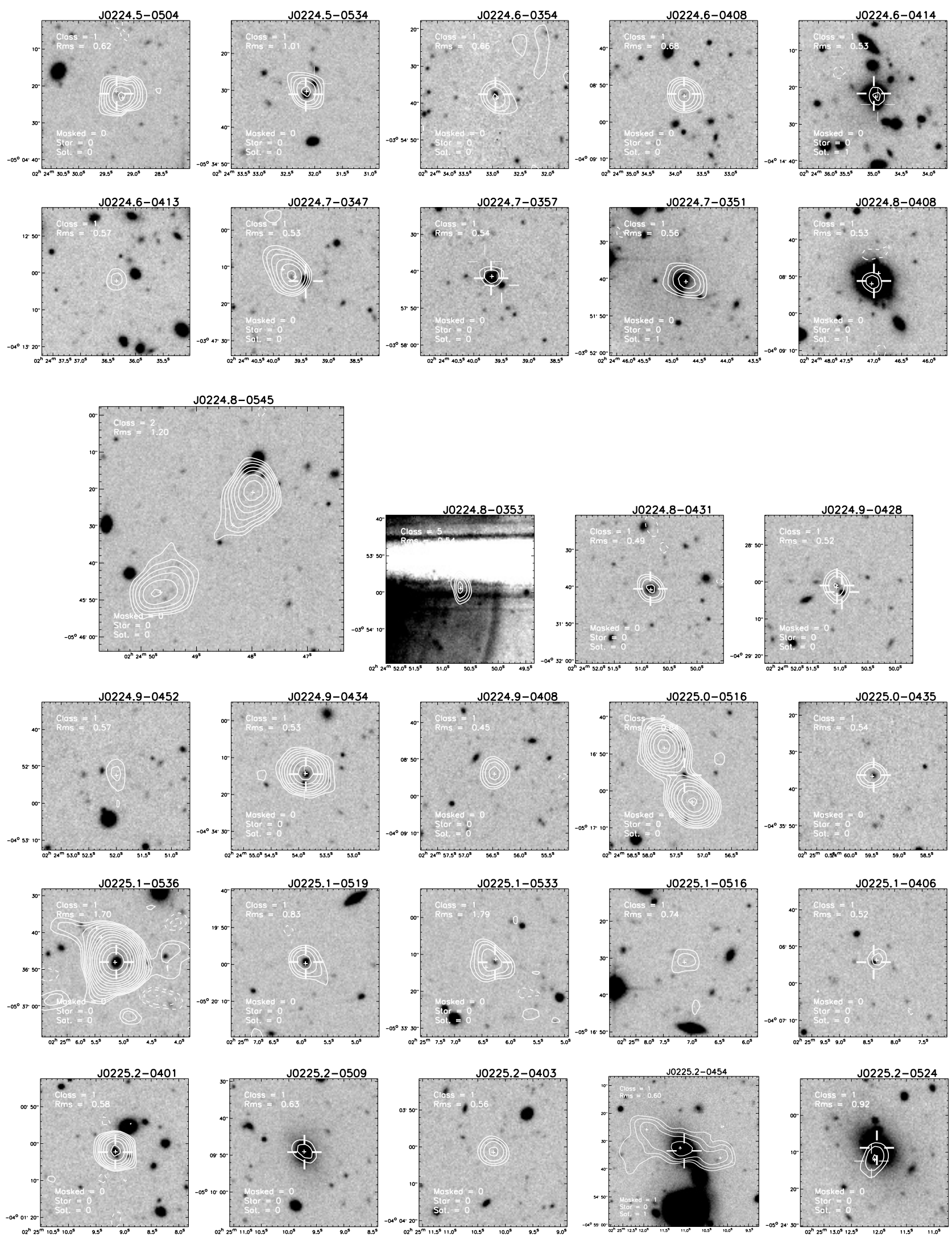

Fig. E.1. continued. 
C. Tasse et al.: The optical and infrared properties of low-frequency radio sources in the XMM-LSS field, Online Material p 30
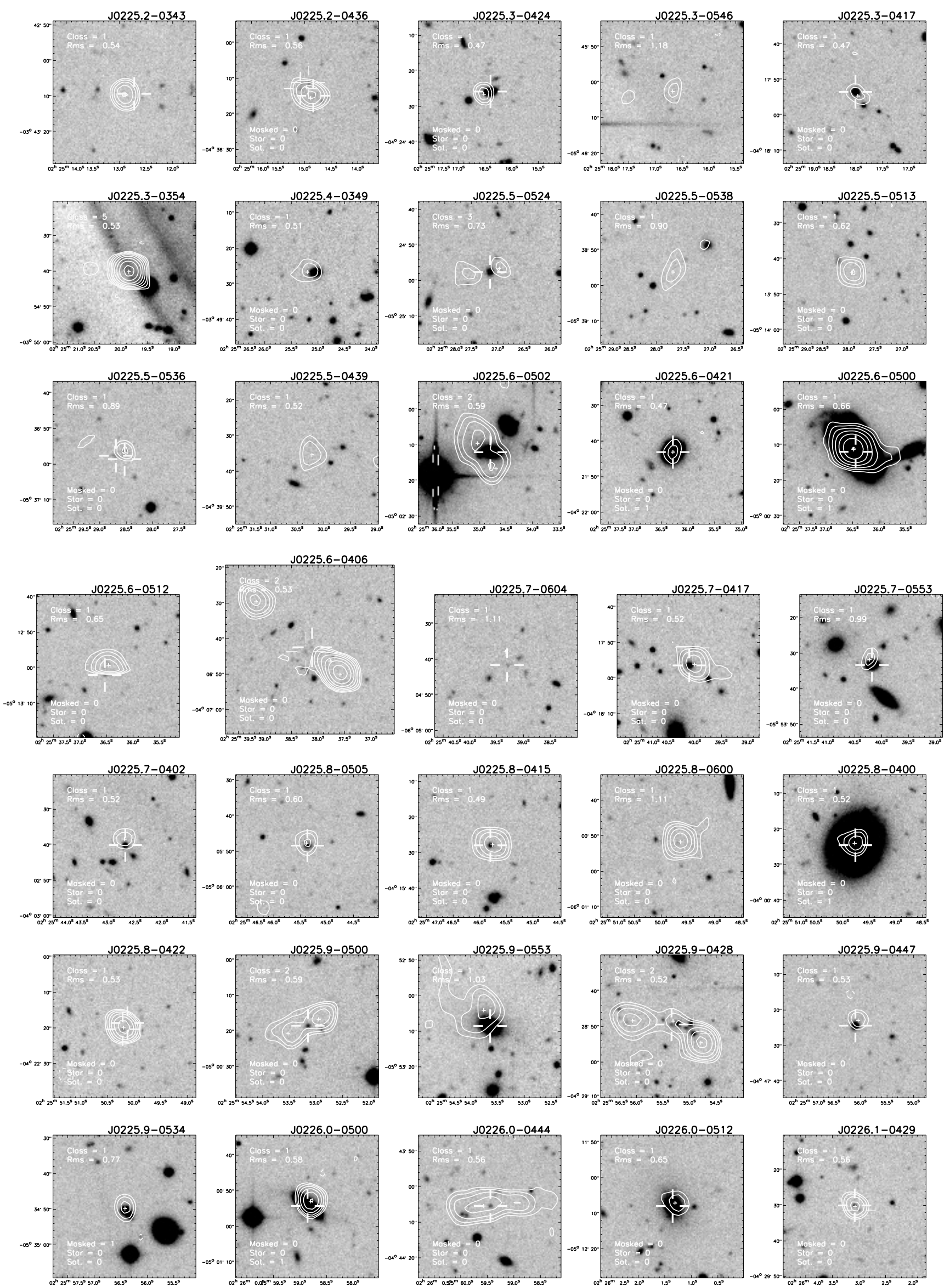

Fig. E.1. continued. 
C. Tasse et al.: The optical and infrared properties of low-frequency radio sources in the XMM-LSS field, Online Material p 31
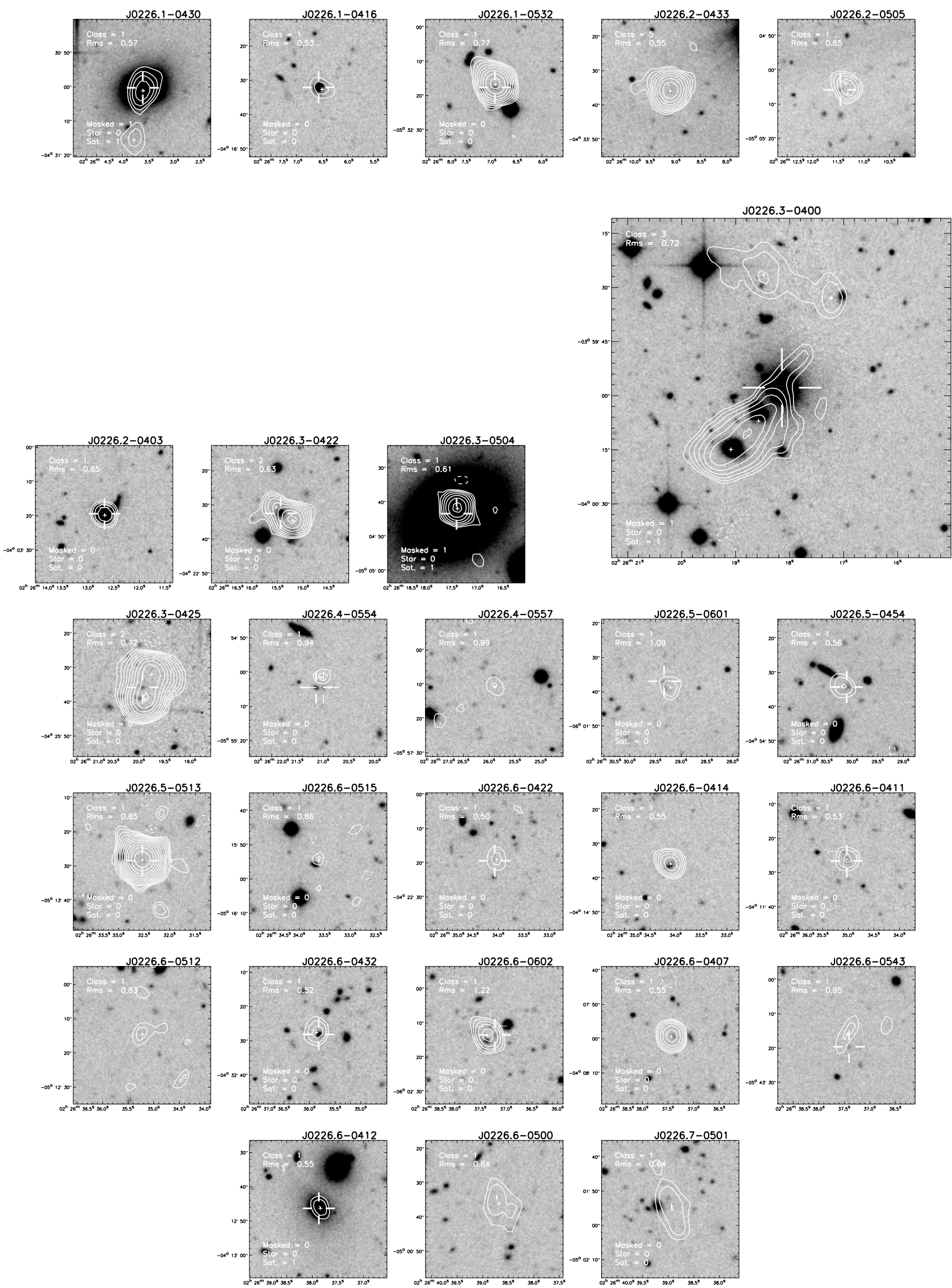

Fig. E.1. continued. 
C. Tasse et al.: The optical and infrared properties of low-frequency radio sources in the XMM-LSS field, Online Material p 32
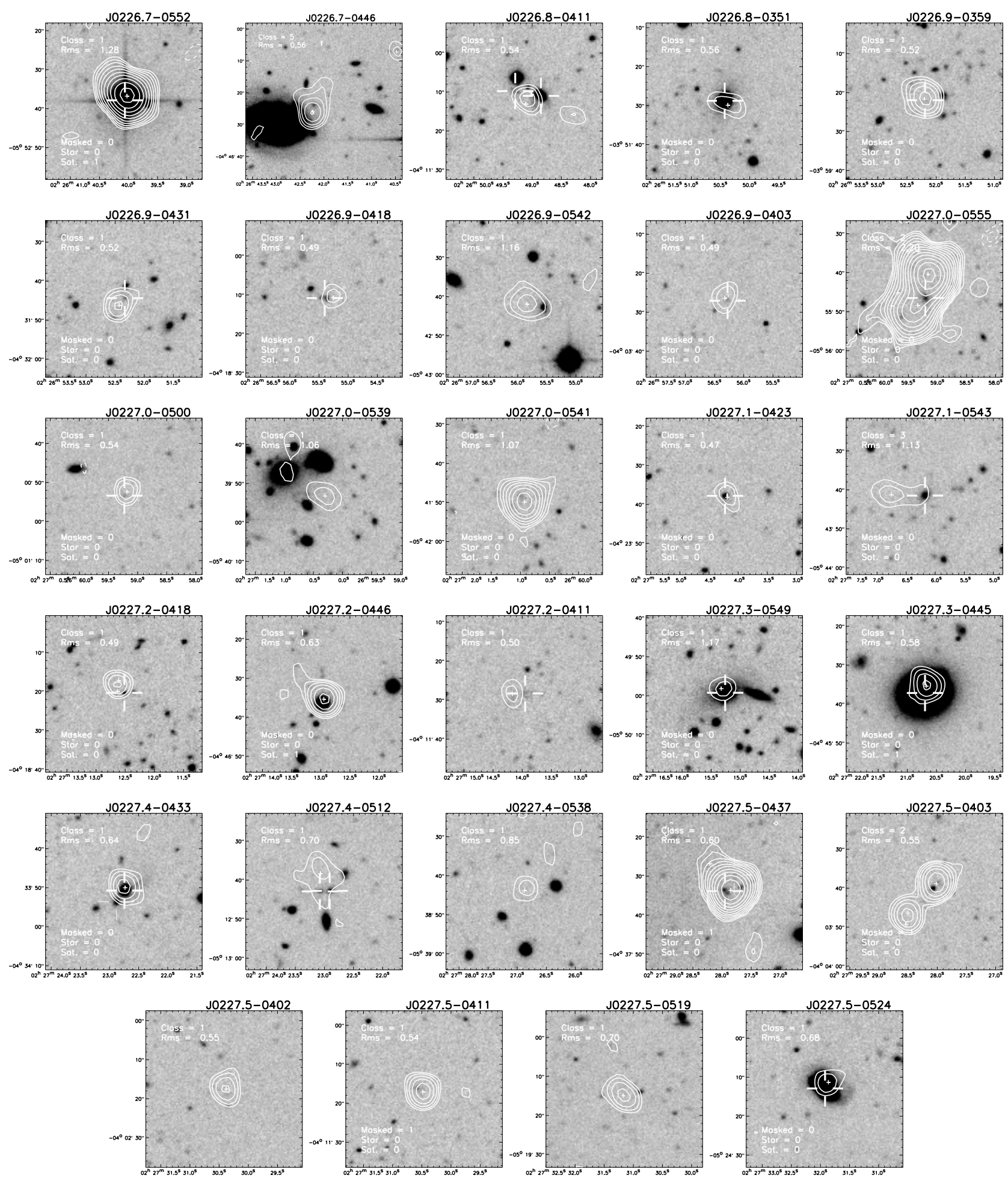

Fig. E.1. continued. 
C. Tasse et al.: The optical and infrared properties of low-frequency radio sources in the XMM-LSS field, Online Material p 33
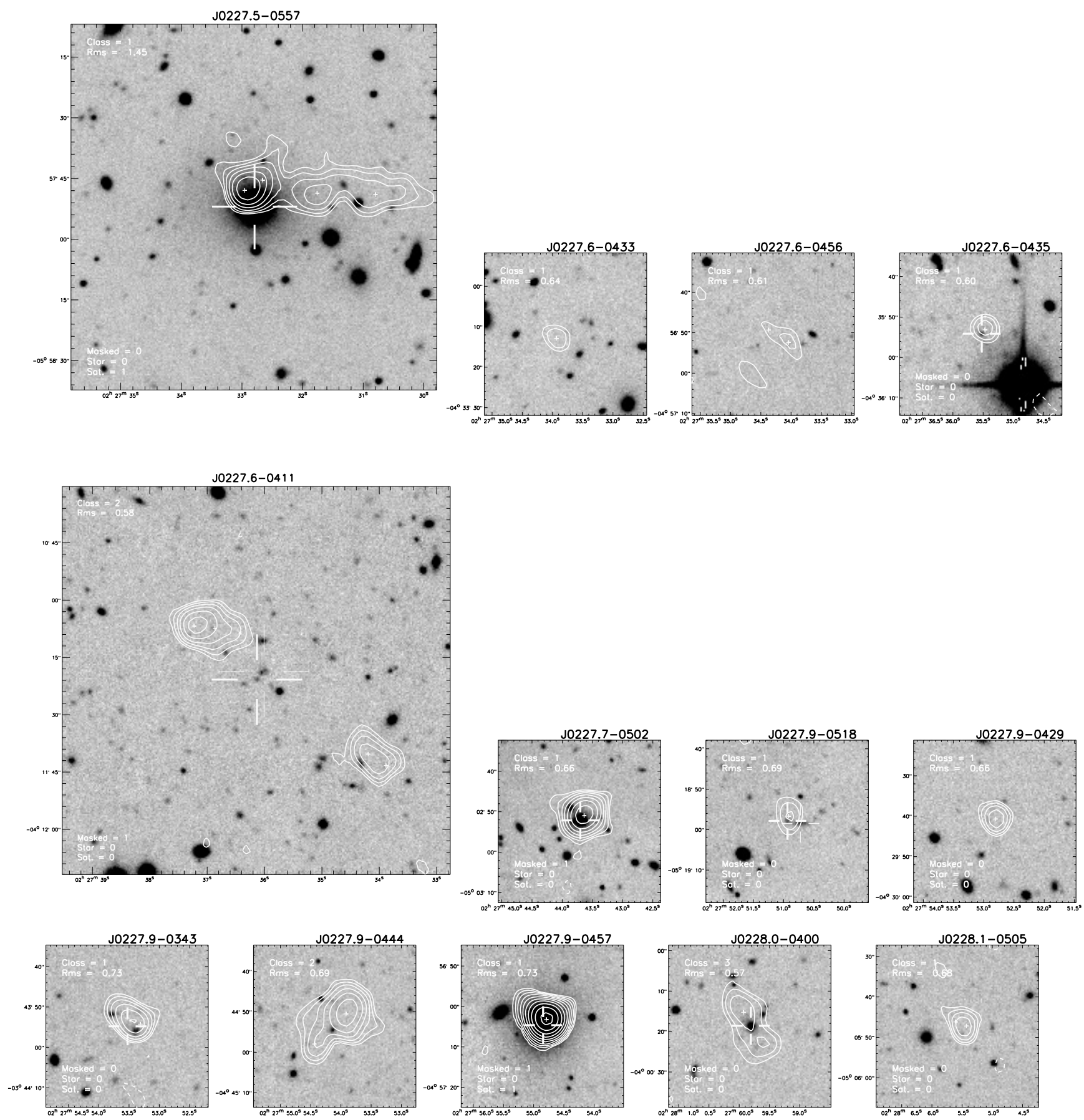

Fig. E.1. continued. 
C. Tasse et al.: The optical and infrared properties of low-frequency radio sources in the XMM-LSS field, Online Material p 34

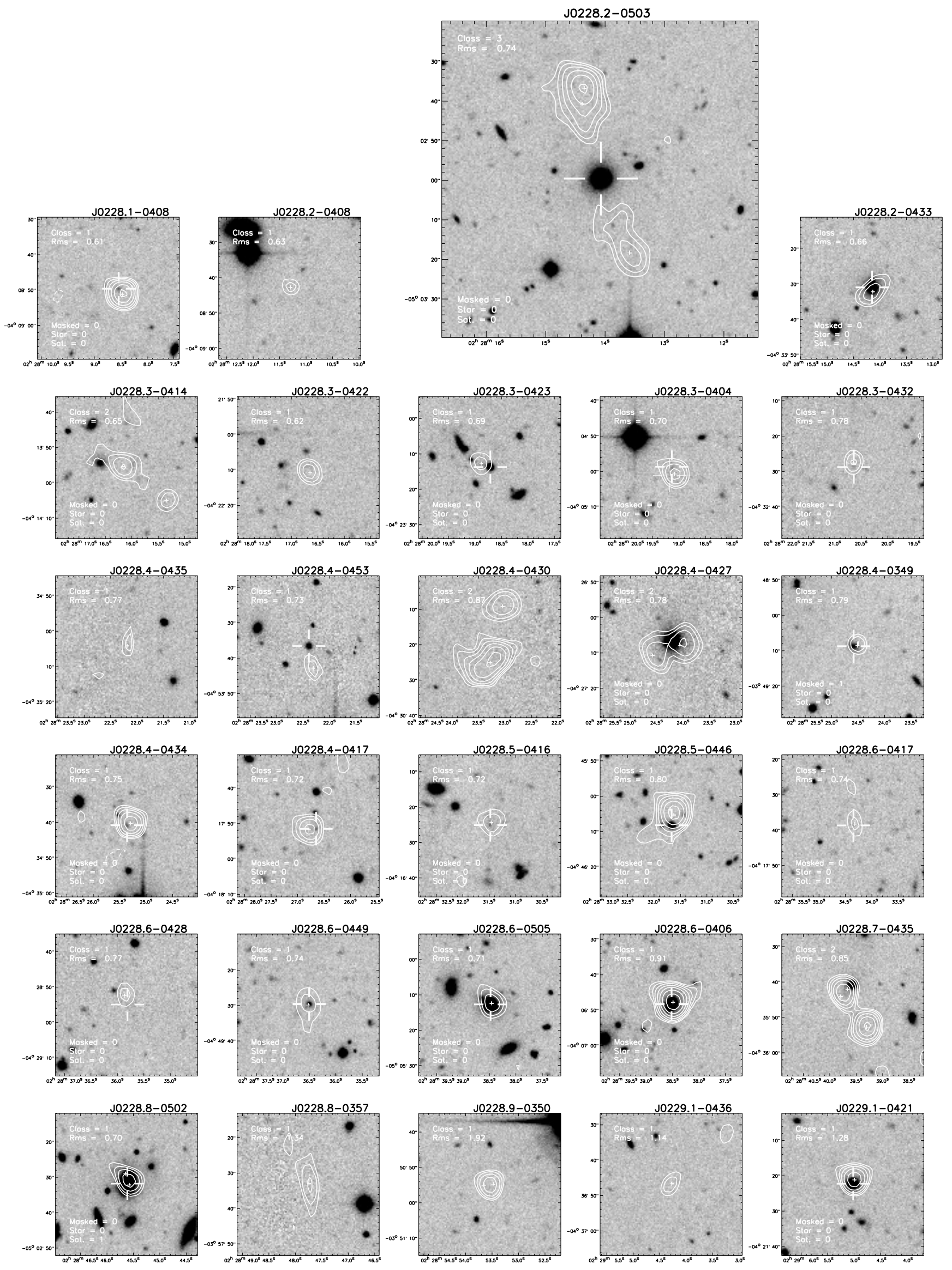

Fig. E.1. continued. 
C. Tasse et al.: The optical and infrared properties of low-frequency radio sources in the XMM-LSS field, Online Material p 35
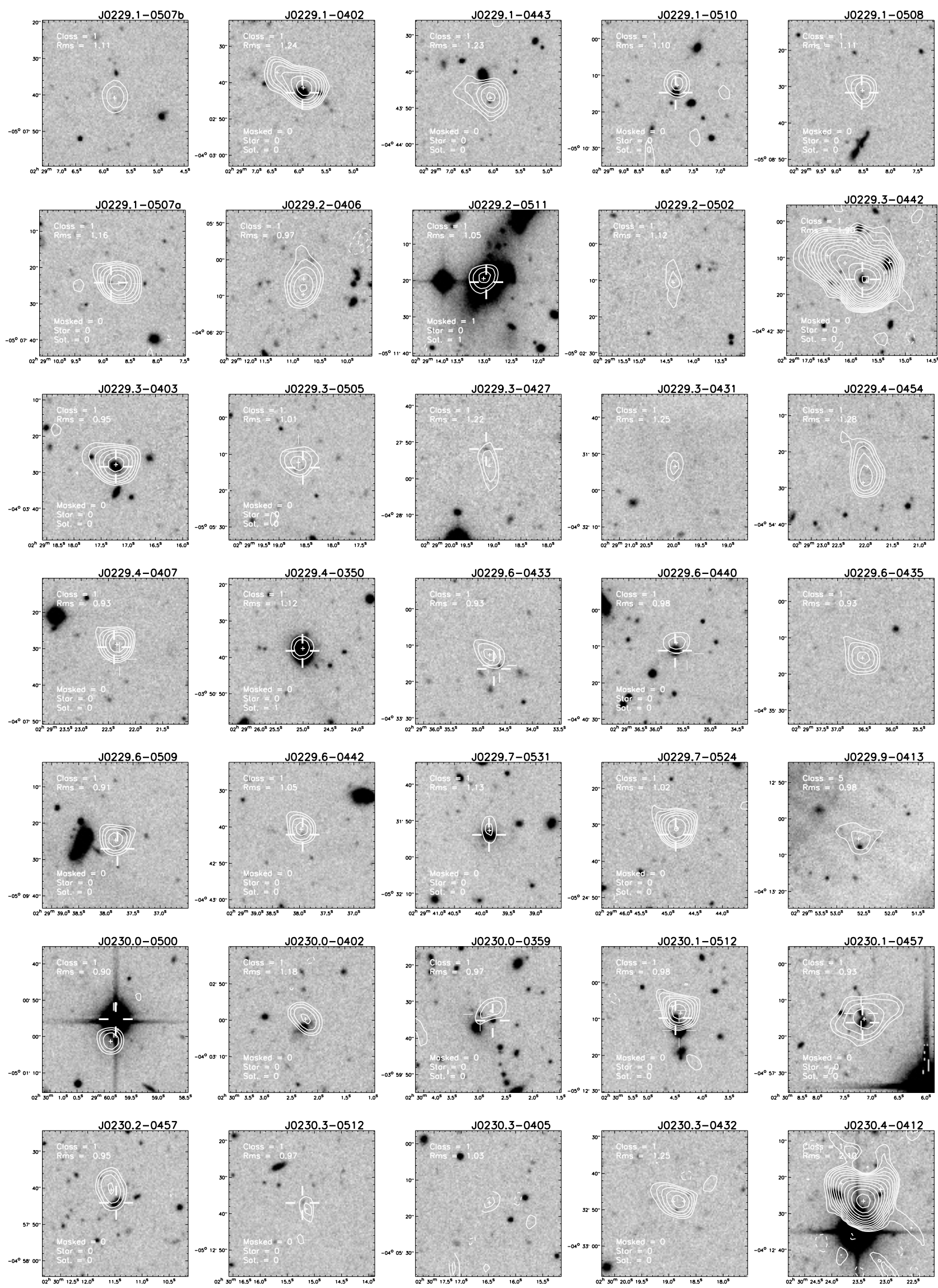

Fig. E.1. continued. 
C. Tasse et al.: The optical and infrared properties of low-frequency radio sources in the XMM-LSS field, Online Material p 36
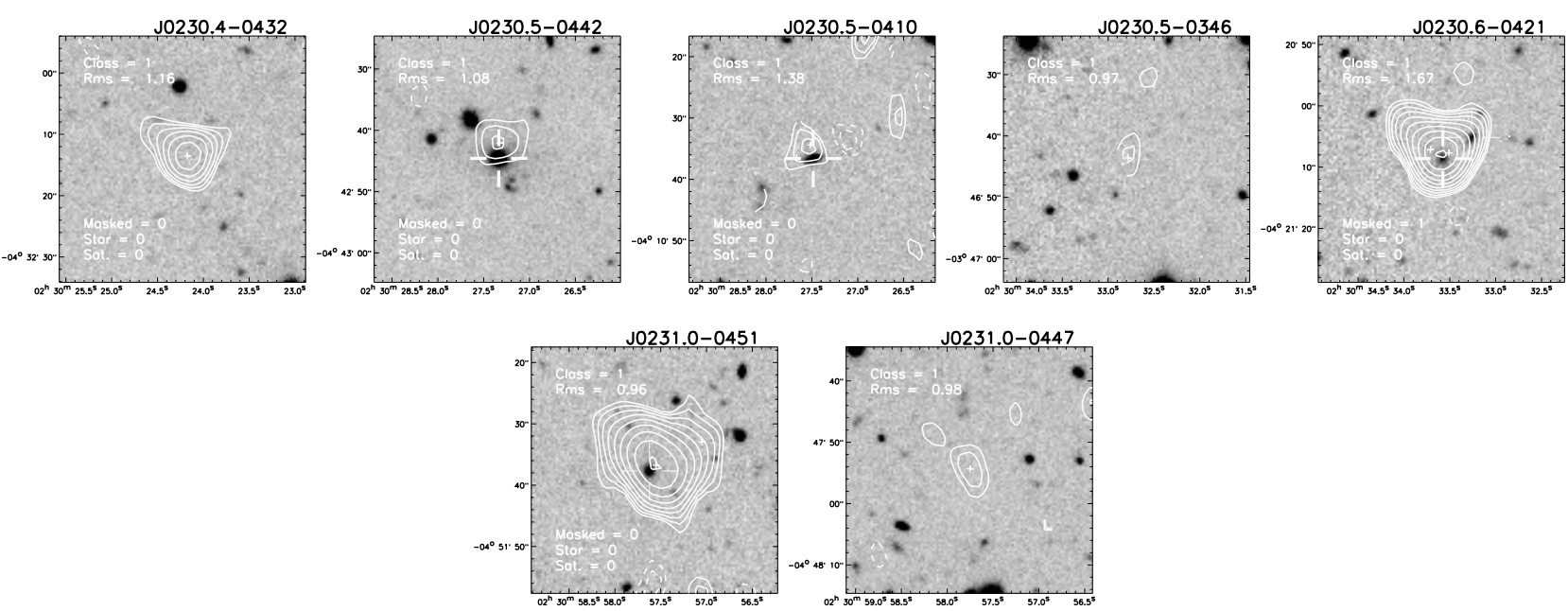

Fig. E.1. continued. 
C. Tasse et al.: The optical and infrared properties of low-frequency radio sources in the XMM-LSS field, Online Material p 37
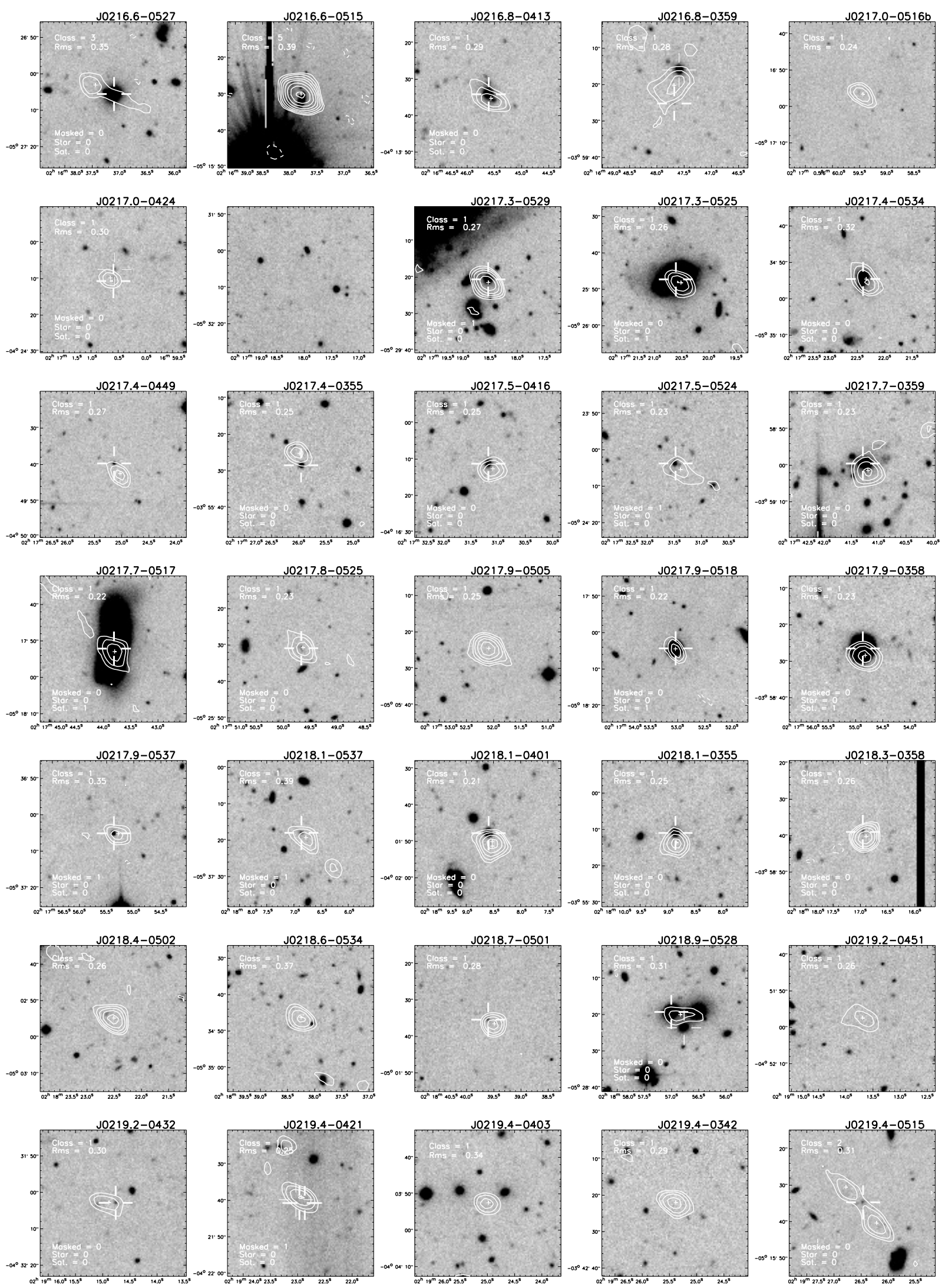

Fig. E.2. Greyscale: the $i$-band image. Contours: the $610 \mathrm{MHz}$ contours drawn at levels of $3 \sigma_{\text {local }} \times 1,1.4,2,2.8,4, \ldots$ The local noise $\sigma_{\text {local }}$ appears in each overlay, and is in units of mJy beam ${ }^{-1}$. The largest cross indicates the position of the optical identification with the highest identification probability, $p_{\max }$. The thickness of the other crosses scales as $p / p_{\max }$ where $p$ is the probability of identification of the given source. Small crosses indicate the positions of the Gaussian fitting components appearing in the Tasse et al. (2007) source list, used for the centroïd position calculation. On the bottom left corner of each image, the radio source morphological classification is given, as well as the flag status of the optical host in the optical catalog: Masked $=1$ if the objects is masked, Star $=1$ if it is point-like, and Sat. $=1$ if it is saturated. 
C. Tasse et al.: The optical and infrared properties of low-frequency radio sources in the XMM-LSS field, Online Material p 38
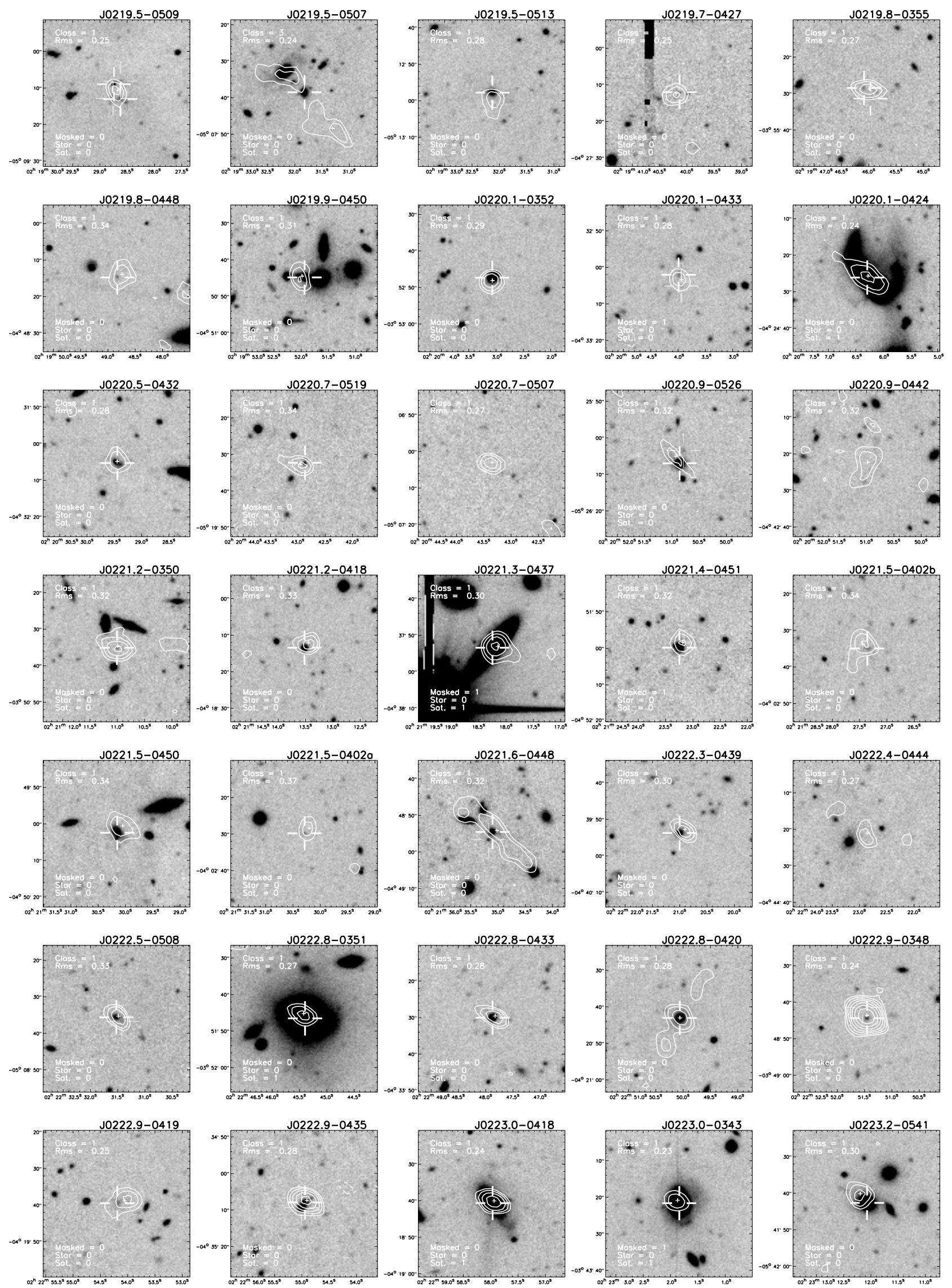

Fig. E.2. continued. 
C. Tasse et al.: The optical and infrared properties of low-frequency radio sources in the XMM-LSS field, Online Material p 39
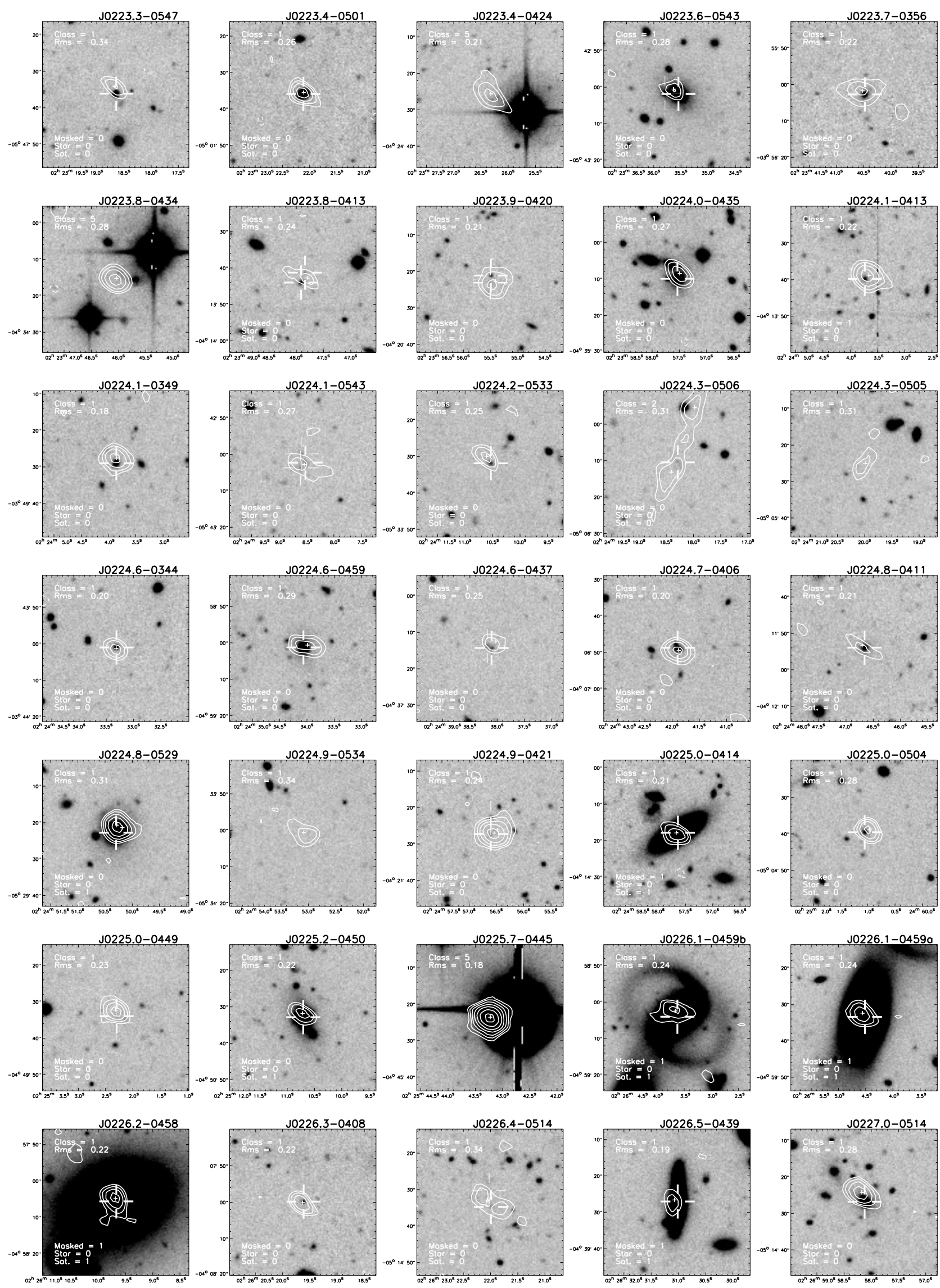

Fig. E.2. continued. 
C. Tasse et al.: The optical and infrared properties of low-frequency radio sources in the XMM-LSS field, Online Material p 40
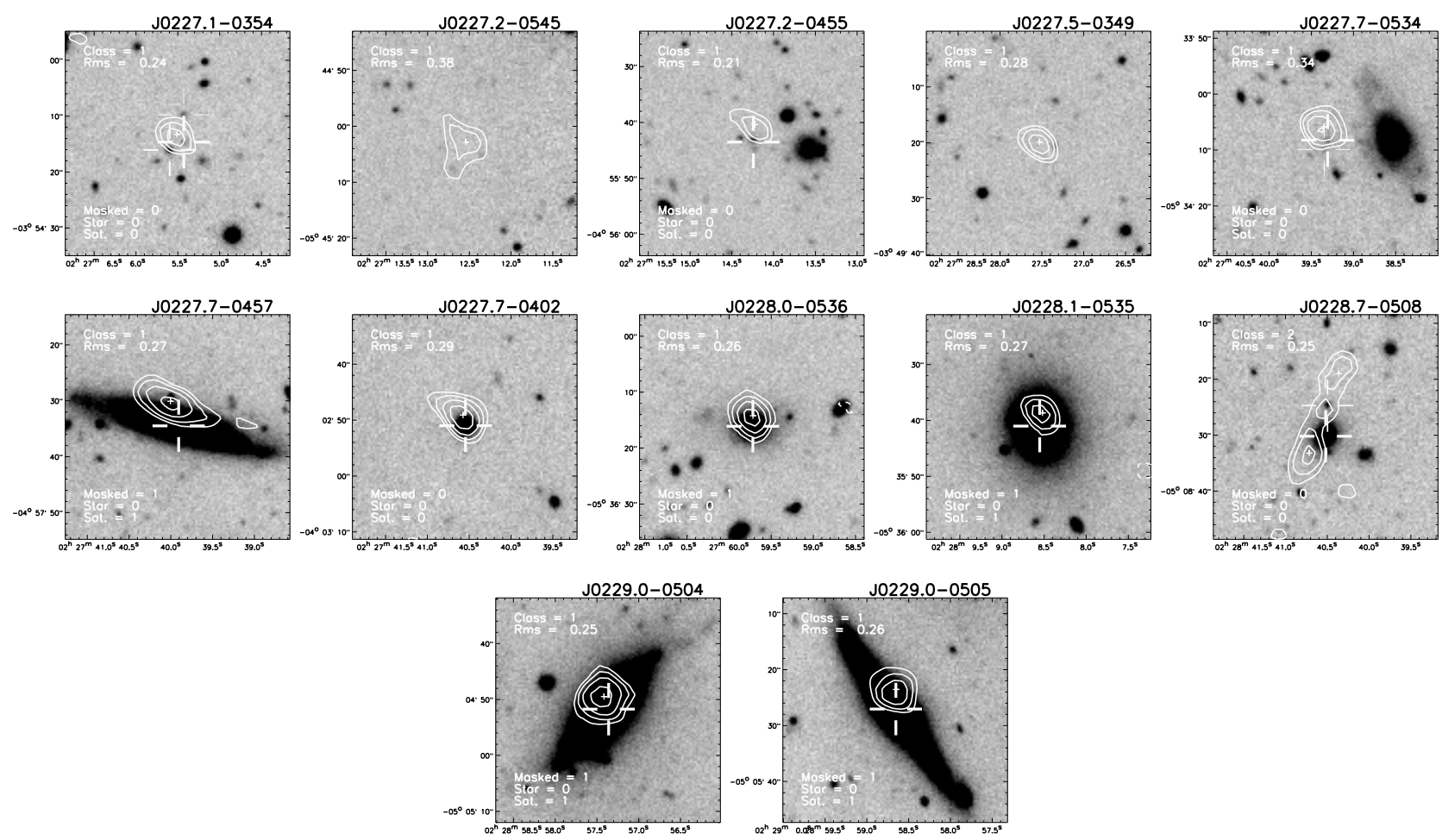

Fig. E.2. continued. 

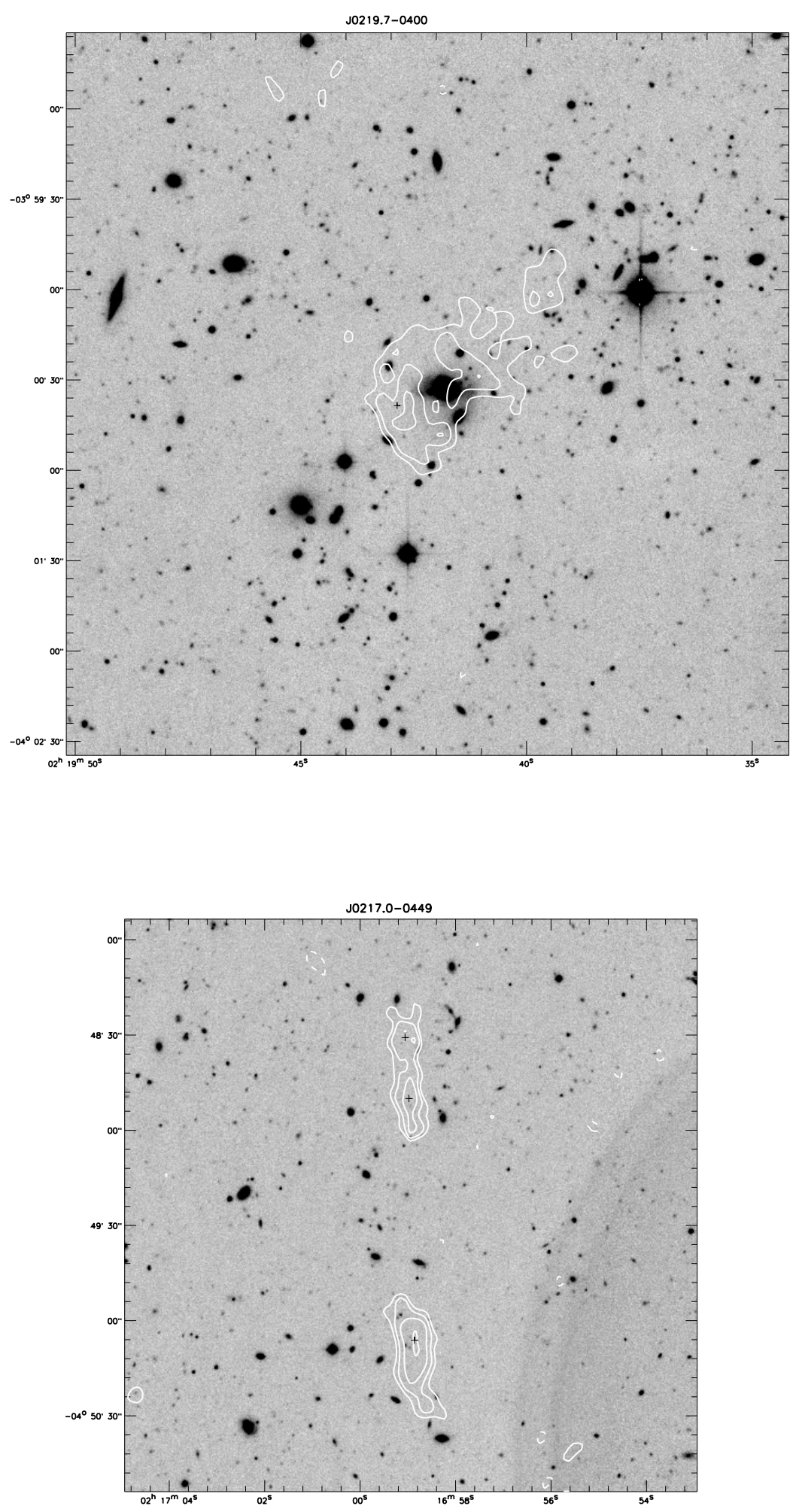

Fig. E.3. Greyscale: the $i$-band image. Contours: the $325 \mathrm{MHz}$ contours drawn at levels of $3 \sigma_{\text {local }} \times 1,1.4,2,2.8,4, \ldots$ Small crosses indicate the positions of the Gaussian fitting components appearing in the Tasse et al. (2006) source list, used for the centroïd position calculation. The top panel shows the radio halo/relic candidate reported in Tasse et al. (2006). The radio source in the lower panel has a spectral index $\alpha_{1400}^{325}=-1.65$. 